Supporting Information for

Cu-catalyzed Dimerization of Indole Derived Oxime Acetate for

\title{
Synthesis of Biimidazo[1,2-a]indoles
}

Tao Xie, ${ }^{+}$Qi-Bang Sui, ${ }^{+a}$ Lu-Zhe Qin, ${ }^{+}{ }^{a}$ Xiaoan Wen, ${ }^{a}$ Hongbin Sun, ${ }^{a}$ Qing-Long

$$
\mathrm{Xu}^{*} a \text { and Le Zhen }{ }^{*}
$$

a Jiangsu Key Laboratory of Drug Discovery for Metabolic Diseases and State Key

Laboratory of Natural Medicines, Center of Drug Discovery, China Pharmaceutical University, 24 Tongjia Xiang, Nanjing 210009, China

${ }^{b}$ Key Laboratory of Drug Metabolism and Pharmacokinetics, China Pharmaceutical University, 24 Tongjia Xiang, Nanjing 210009, China

${ }^{*}$ Corresponding author.

E-mail: qlxu@cpu.edu.cn; i_m_zhenle@163.com

\section{Table of contents}

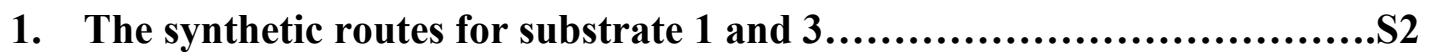

2. NMR Spectra of substrate $1 、 3$ and product $2 、 4 \ldots \ldots \ldots \ldots \ldots \ldots . . . . . . . . . . . .54$

3. X-ray single crystal for compounds $d l-2 \mathrm{a}$ 、meso-2a and $4 \ldots \ldots \ldots \ldots . . . . . . .578$ 
1. The synthetic routes for substrate 1 and 3 .
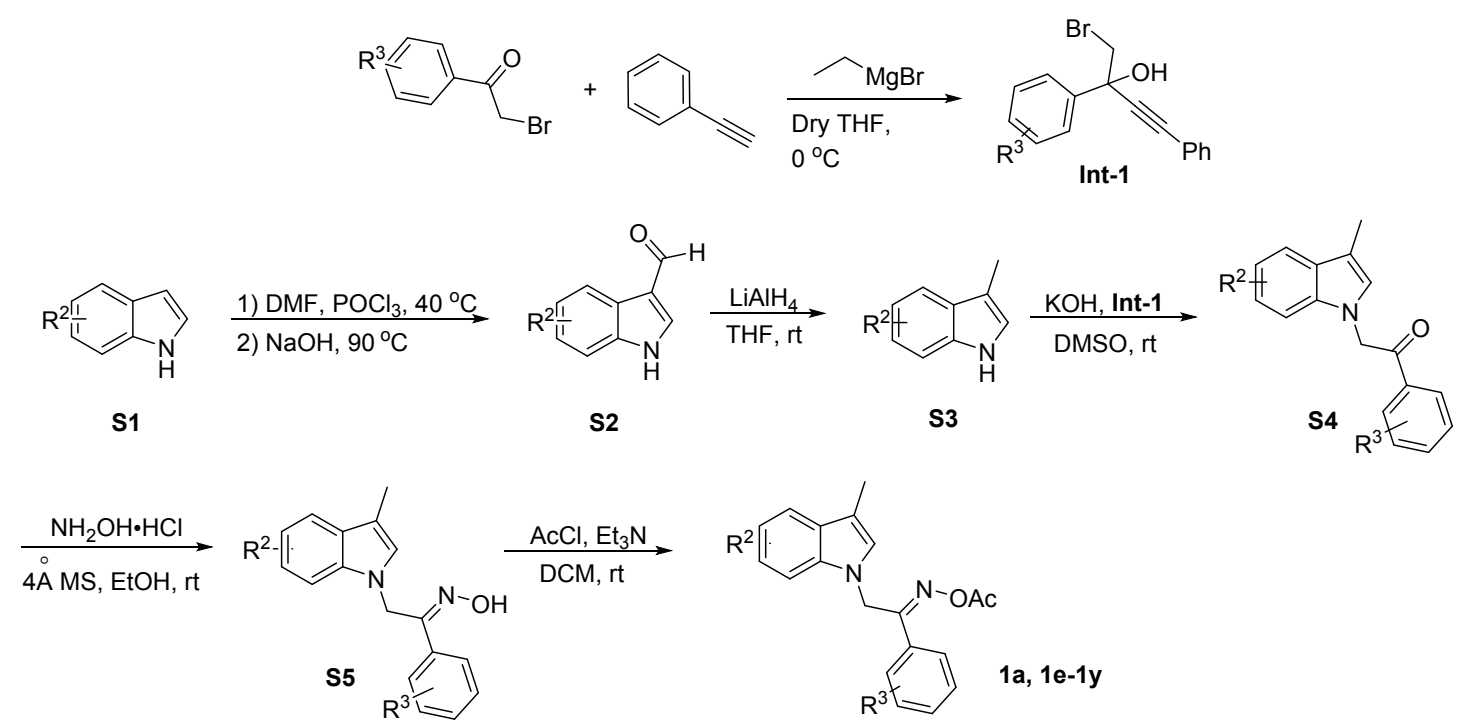

Scheme 1. The route to synthesize compound 1a, 1c-1w.

The strategy for making $\mathbf{S 4}$ was conducted by the following reference (Org. Biomol. Chem. 2017, 15, 8119-8133).<smiles>[R]Cc1cn(CC(=NO)c2ccccc2)c2ccccc12</smiles><smiles>[R]Cc1cn(C/C(=N\OC(C)C)c2ccccc2)c2ccccc12</smiles>

Scheme 2. The route to synthesize compound $1 \mathrm{~b}, 1 \mathrm{~d}$.<smiles>CC(=O)c1ccccc1OCC(C)C</smiles><smiles>Cc1[nH]c2ccccc2c1C(C)C</smiles><smiles>CC(C)Cc1cn(CC(=O)c2ccccc2)c2ccccc12</smiles>

$\underset{4 \stackrel{\mathrm{A}}{\mathrm{NS}, \mathrm{EtOH}, \mathrm{rt}}}{\stackrel{\mathrm{NH}_{2} \mathrm{OH} \cdot \mathrm{HCl}}{\longrightarrow}}$<smiles>CC(C)c1cn(C/C(=N\O)c2ccccc2)c2ccccc12</smiles>

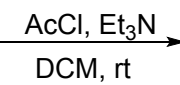<smiles>CC(=O)ON=C(Cn1cc(C(C)C)c2ccccc21)c1ccccc1</smiles>

1c

Scheme 3. The route to synthesize compound $1 \mathrm{c}$. 
<smiles>c1ccc2c3c([nH]c2c1)CCCC3</smiles><smiles></smiles>
DMSO, rt<smiles>O=C(Cn1c2c(c3ccccc31)CCCC2)c1ccccc1</smiles>
$\underset{4 \mathrm{~A} \mathrm{MS}, \mathrm{EtOH}, \mathrm{rt}}{\stackrel{\mathrm{NH}_{2} \mathrm{OH} \cdot \mathrm{HCl}}{\longrightarrow}}$ $\underset{\mathrm{DCM}, \mathrm{rt}}{\stackrel{\mathrm{AcCl}, \mathrm{Et}_{3} \mathrm{~N}}{\longrightarrow}}$<smiles>CC(=O)O/N=C(/Cn1c2c(c3ccccc31)CCCC2)c1ccccc1</smiles><smiles>O/N=C(/Cn1c2c(c3ccccc31)CCCC2)c1ccccc1</smiles>

Scheme 4. The route to synthesize compound 3. 
2. NMR Spectra of substrate 1,3 and product 2,4 .

(E)-2-(3-methyl-1H-indol-1-yl)-1-phenylethan-1-one O-acetyl oxime (1a):

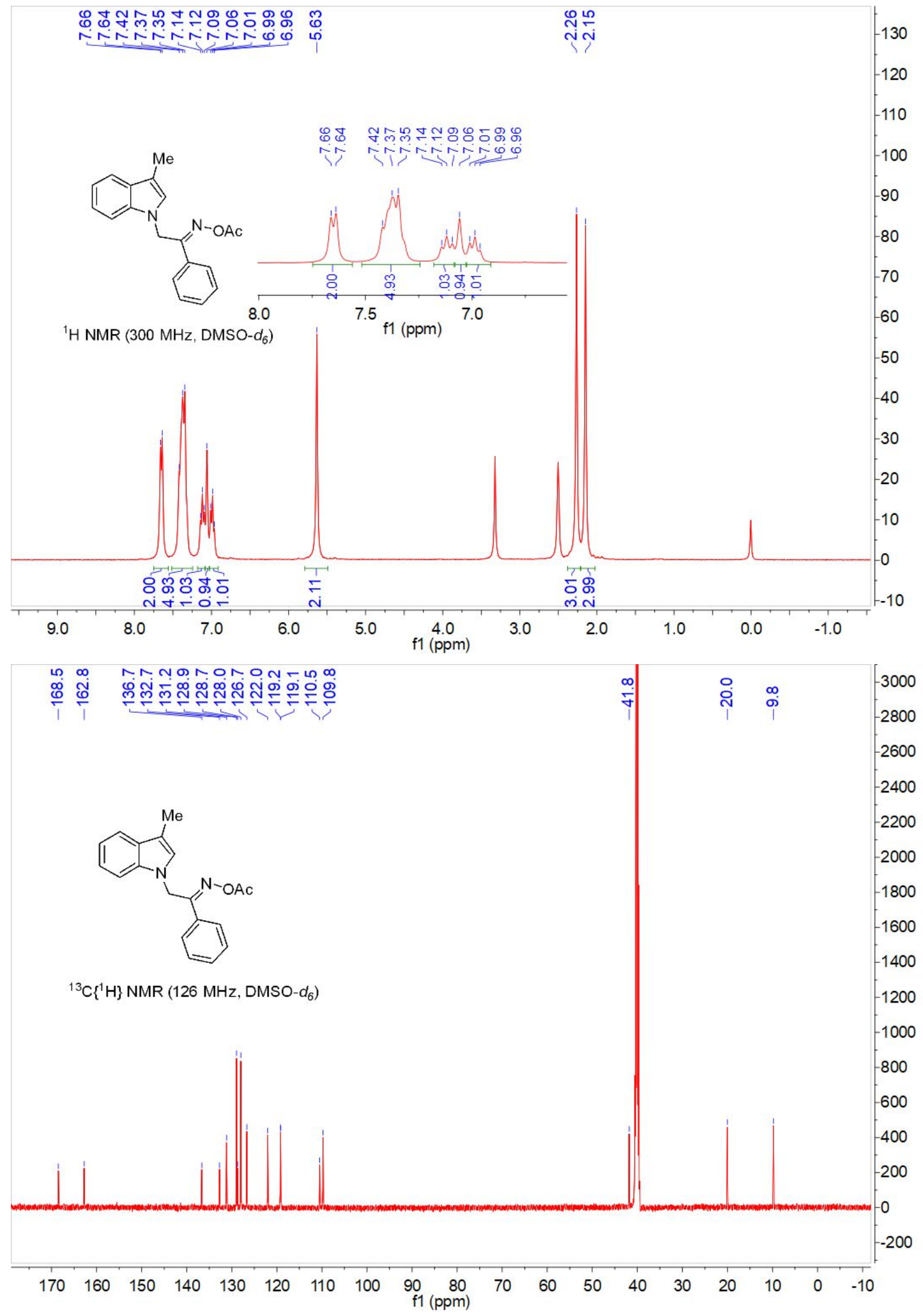


(E)-2-(3-ethyl-1H-indol-1-yl)-1-phenylethan-1-one O-acetyl oxime (1b):

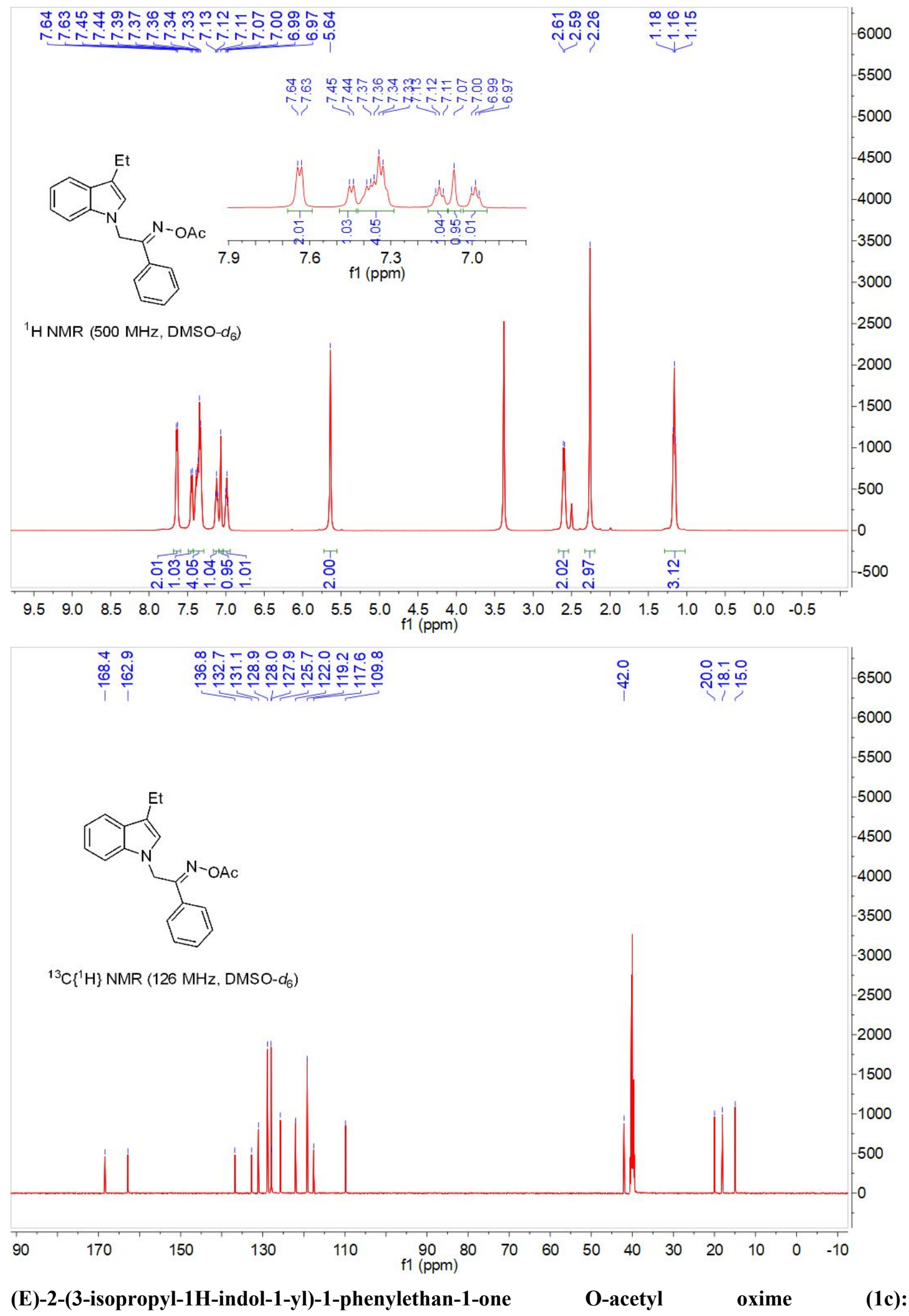




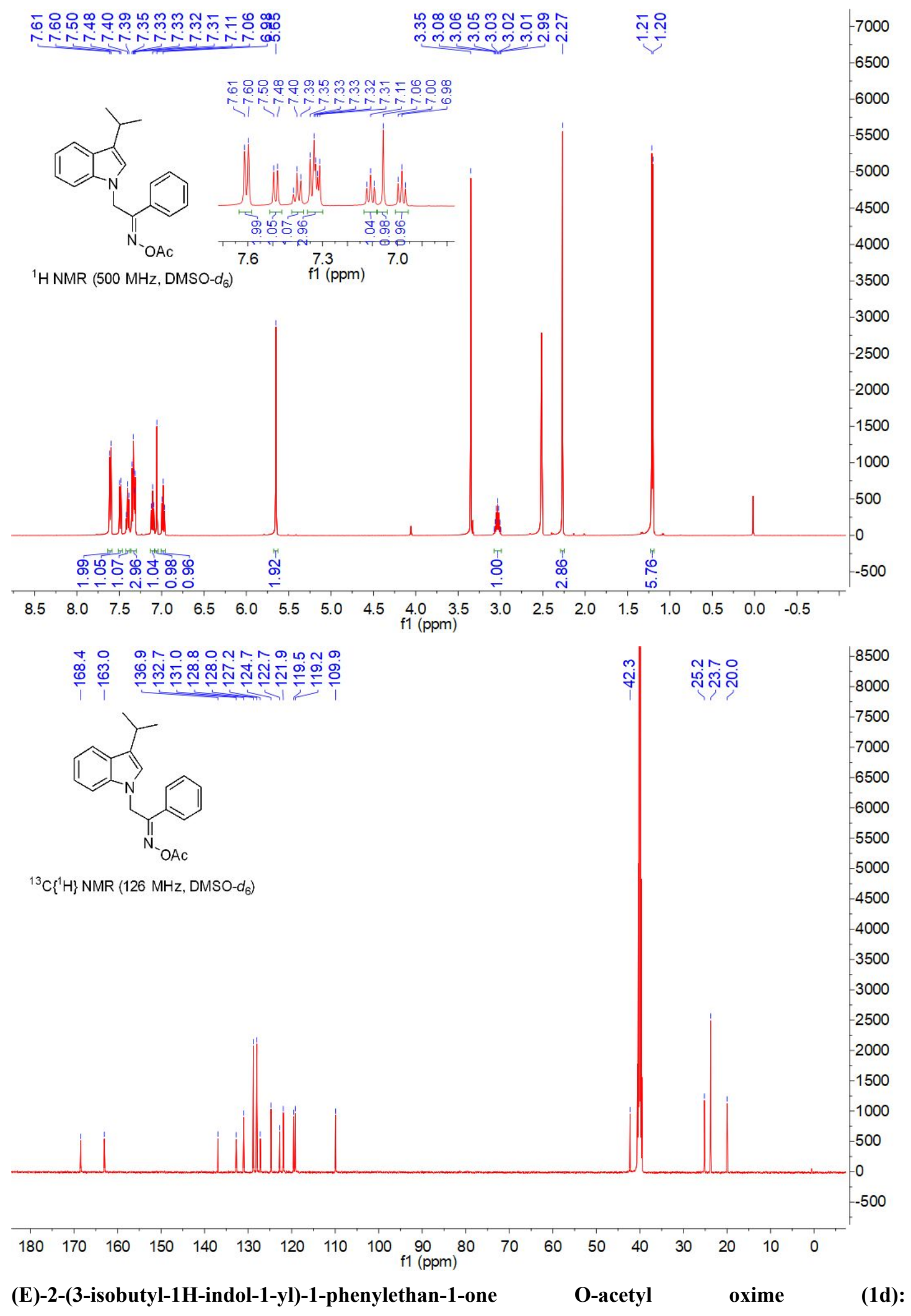




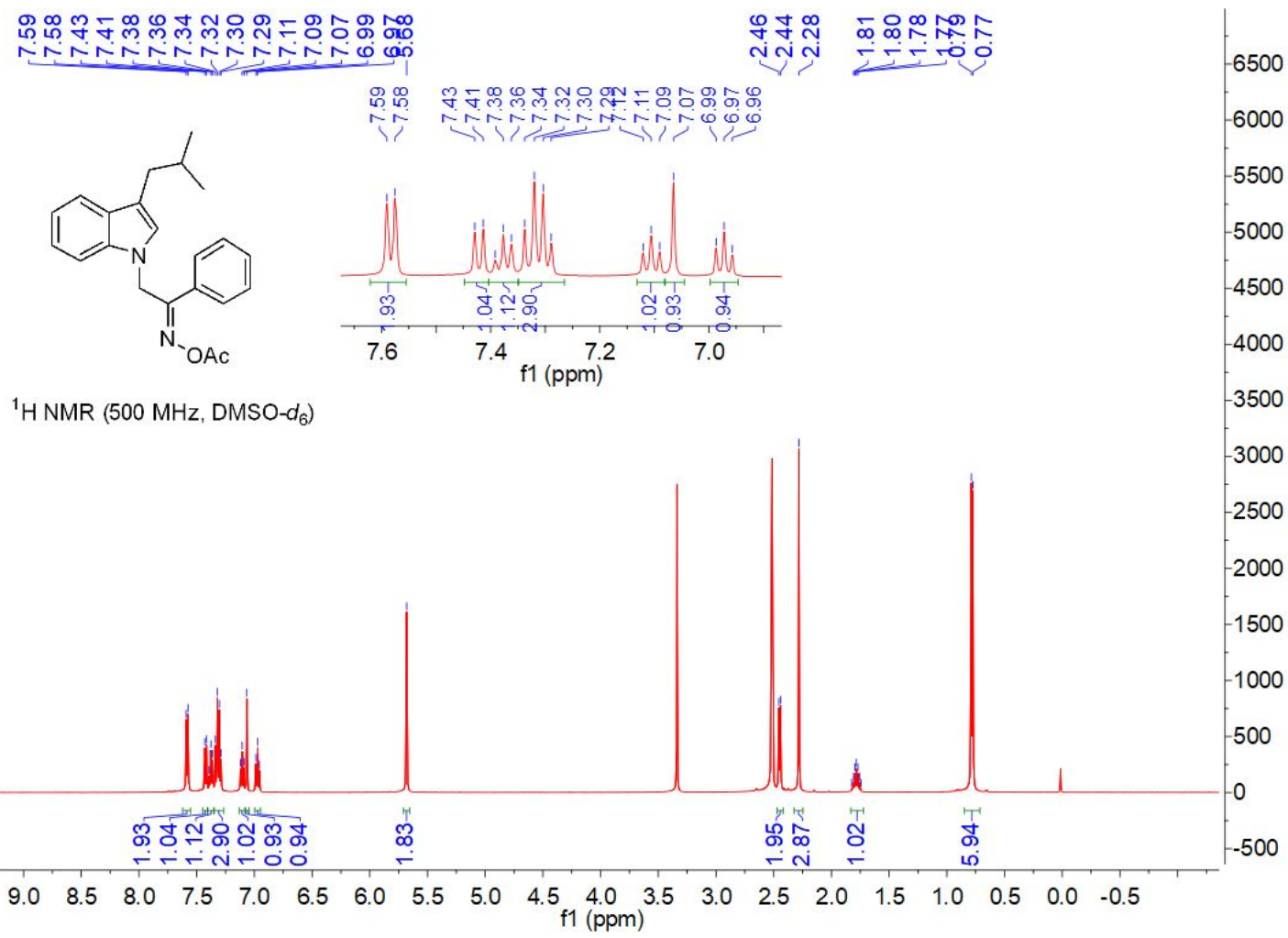

นก) ก

@̋

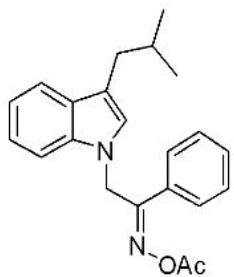

${ }^{13} \mathrm{C}\left\{{ }^{1} \mathrm{H}\right\}$ NMR $\left(126 \mathrm{MHz}, \mathrm{DMSO}-\mathrm{d}_{6}\right)$

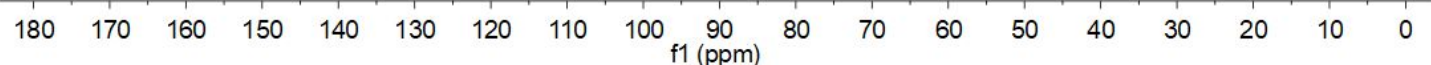


(E)-2-(3,5-dimethyl-1H-indol-1-yl)-1-phenylethan-1-one O-acetyl oxime (1e):

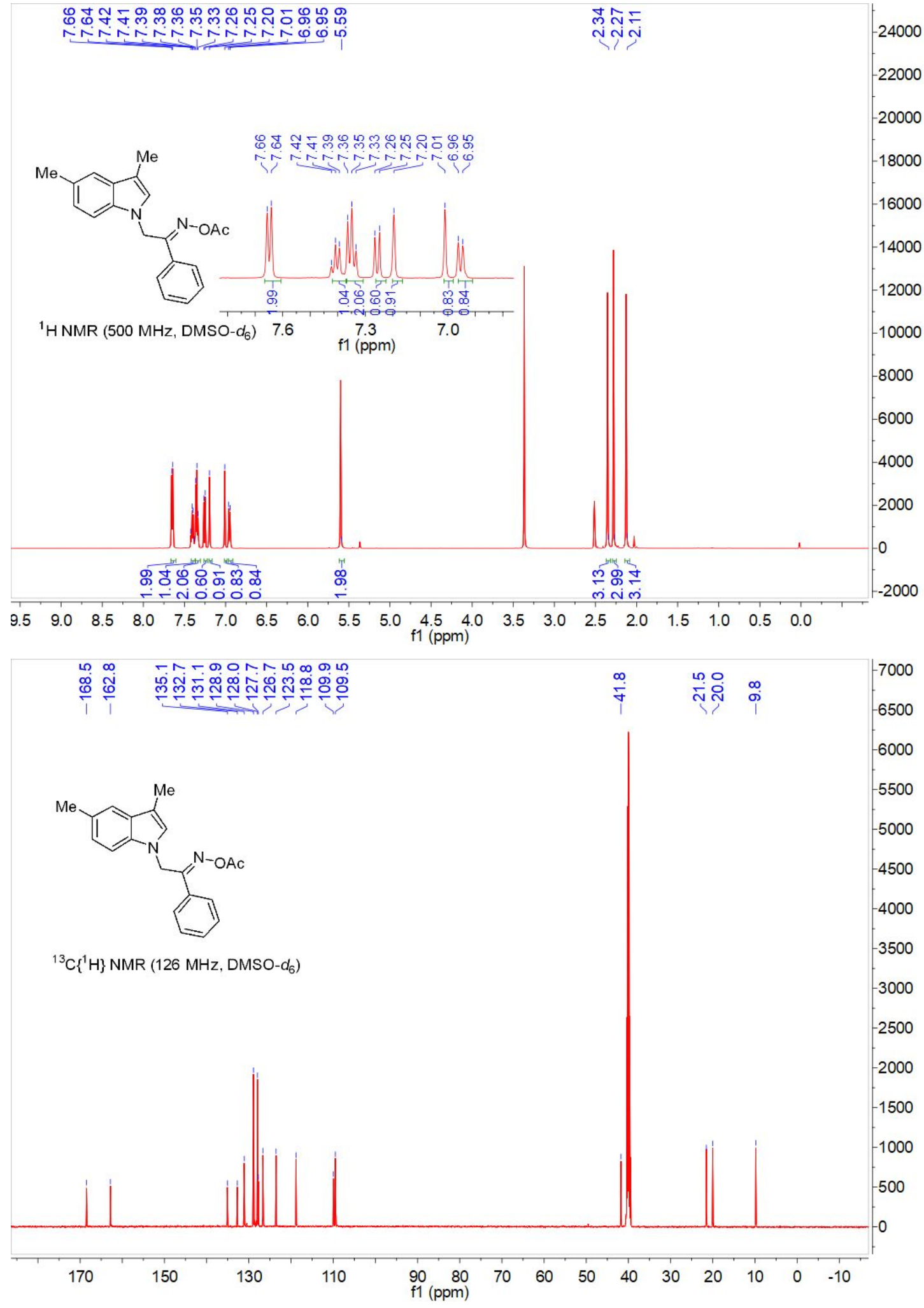


(E)-2-(3,6-dimethyl-1H-indol-1-yl)-1-phenylethan-1-one O-acetyl oxime (1f):

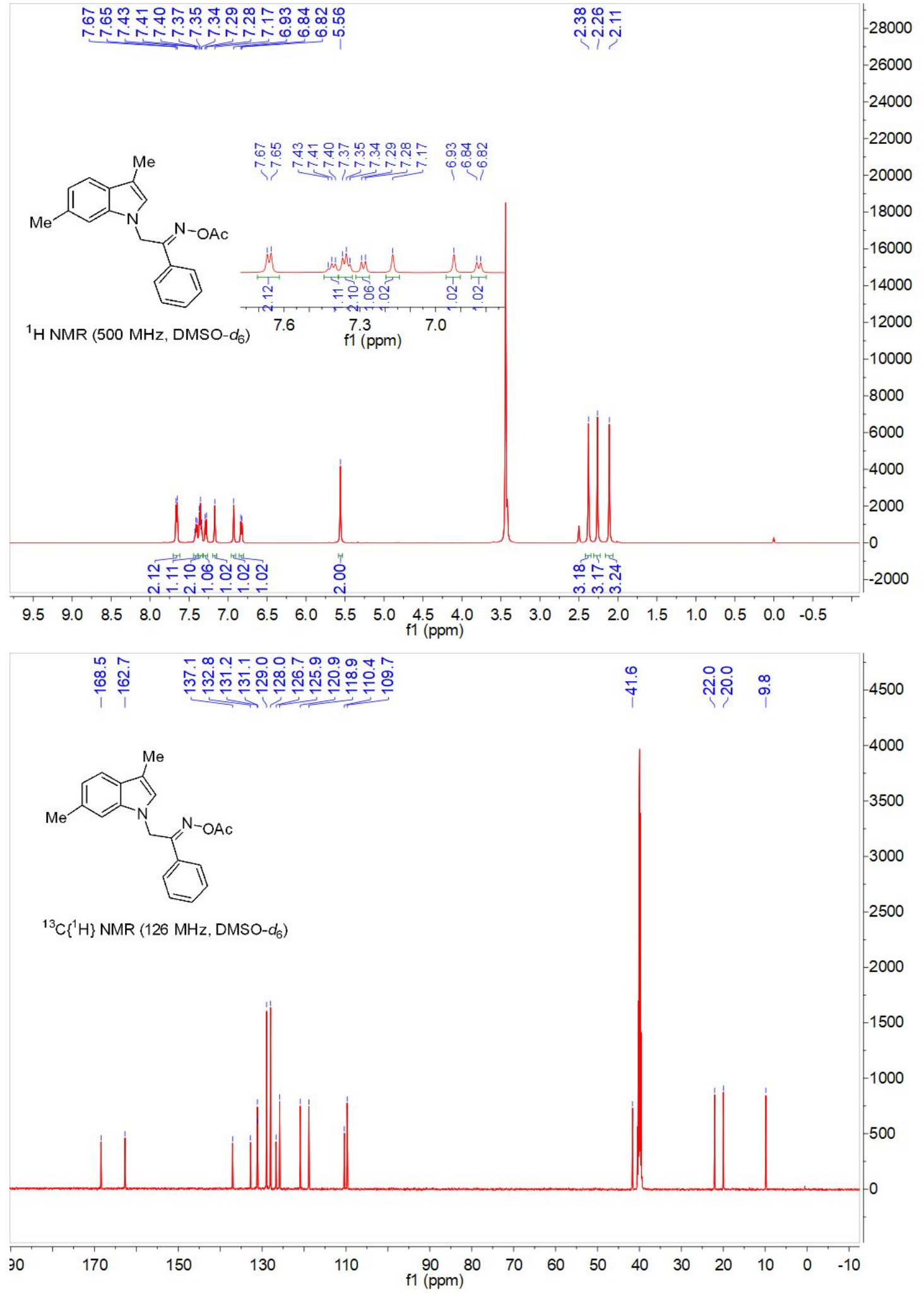


(E)-2-(4-methoxy-3-methyl-1H-indol-1-yl)-1-phenylethan-1-one O-acetyl oxime(1g):

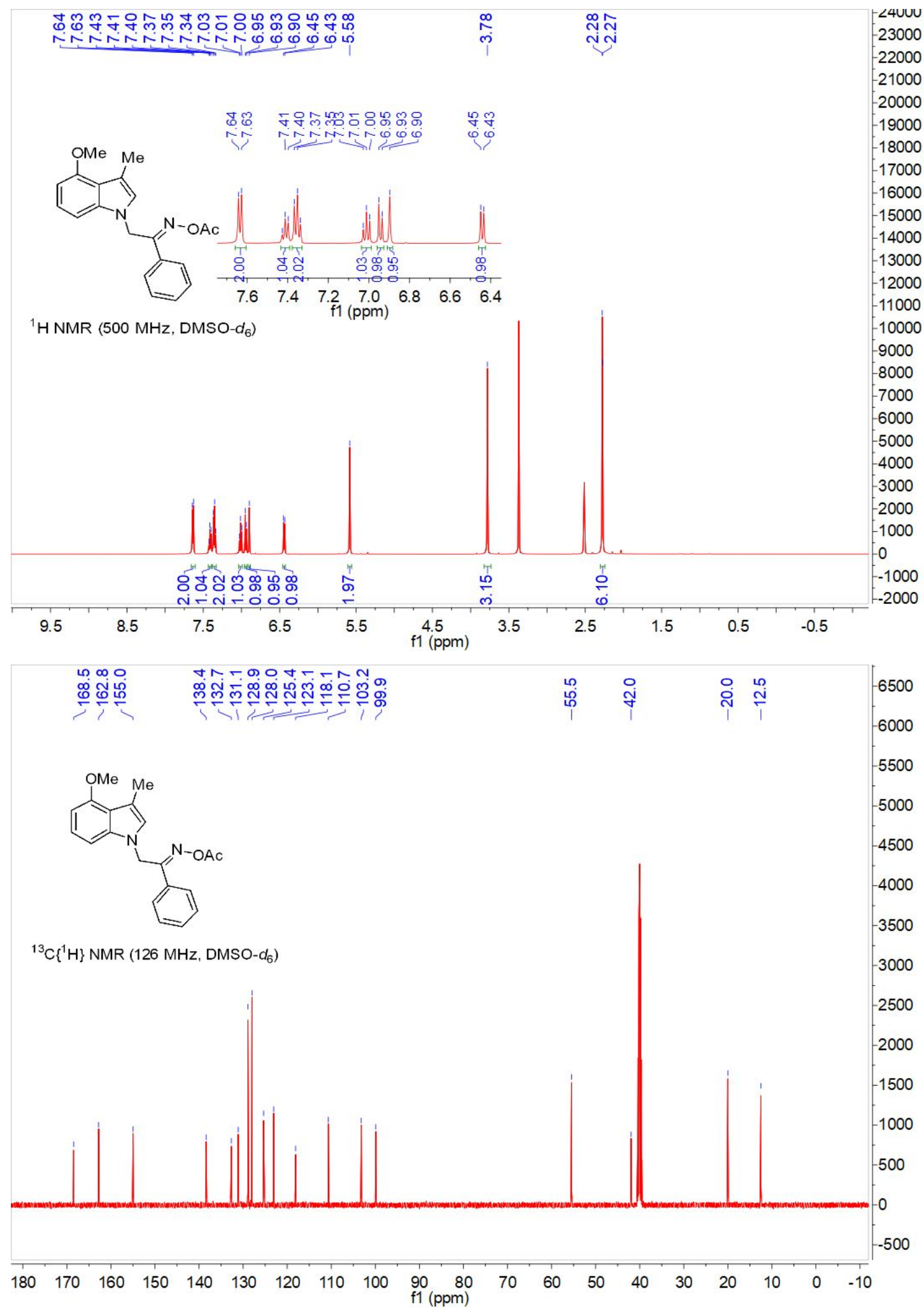


(E)-2-(5-methoxy-3-methyl-1H-indol-1-yl)-1-phenylethan-1-one O-acetyl oxime (1h):

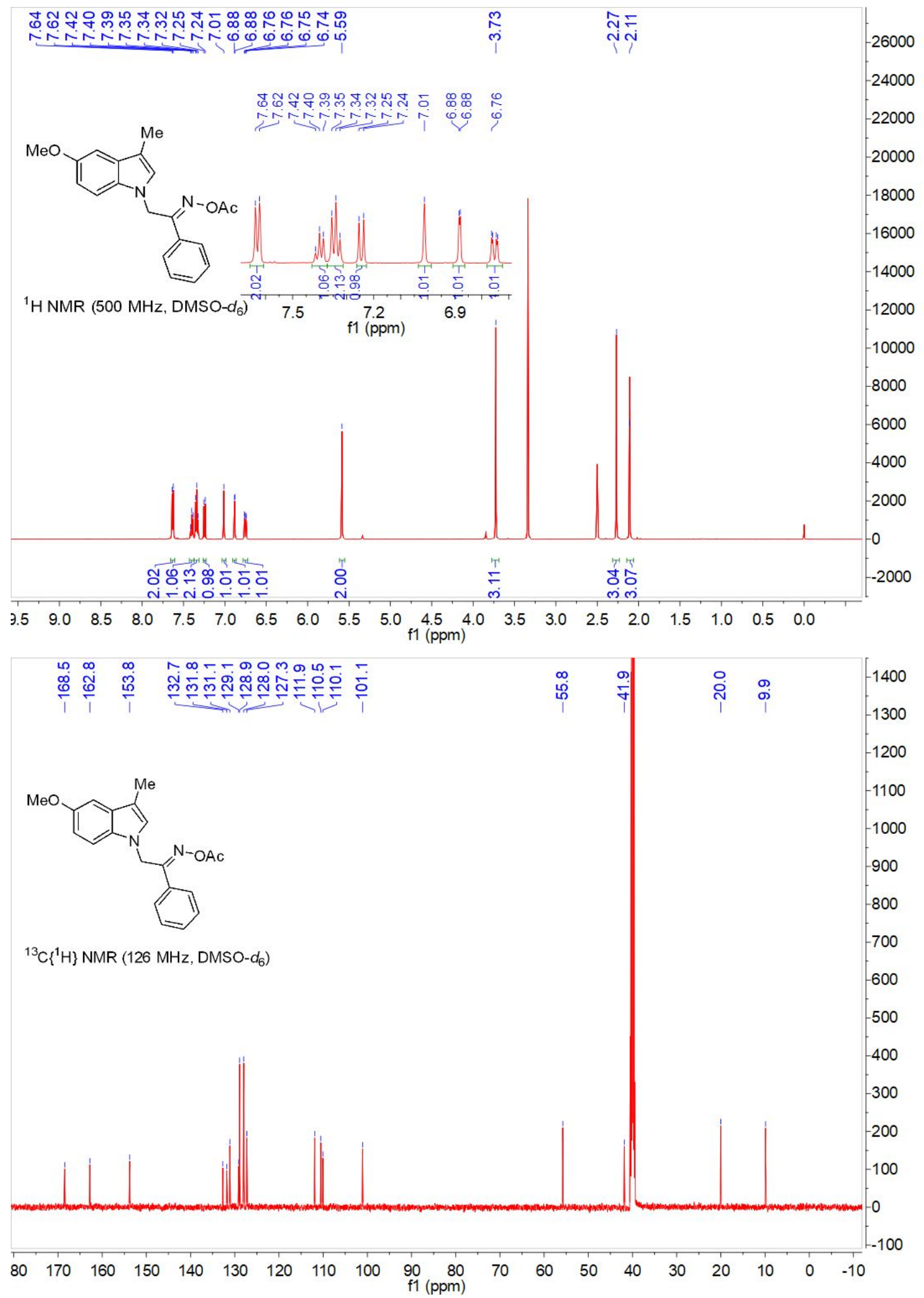


(E)-2-(7-methoxy-3-methyl-1H-indol-1-yl)-1-phenylethan-1-one O-acetyl oxime (1i):

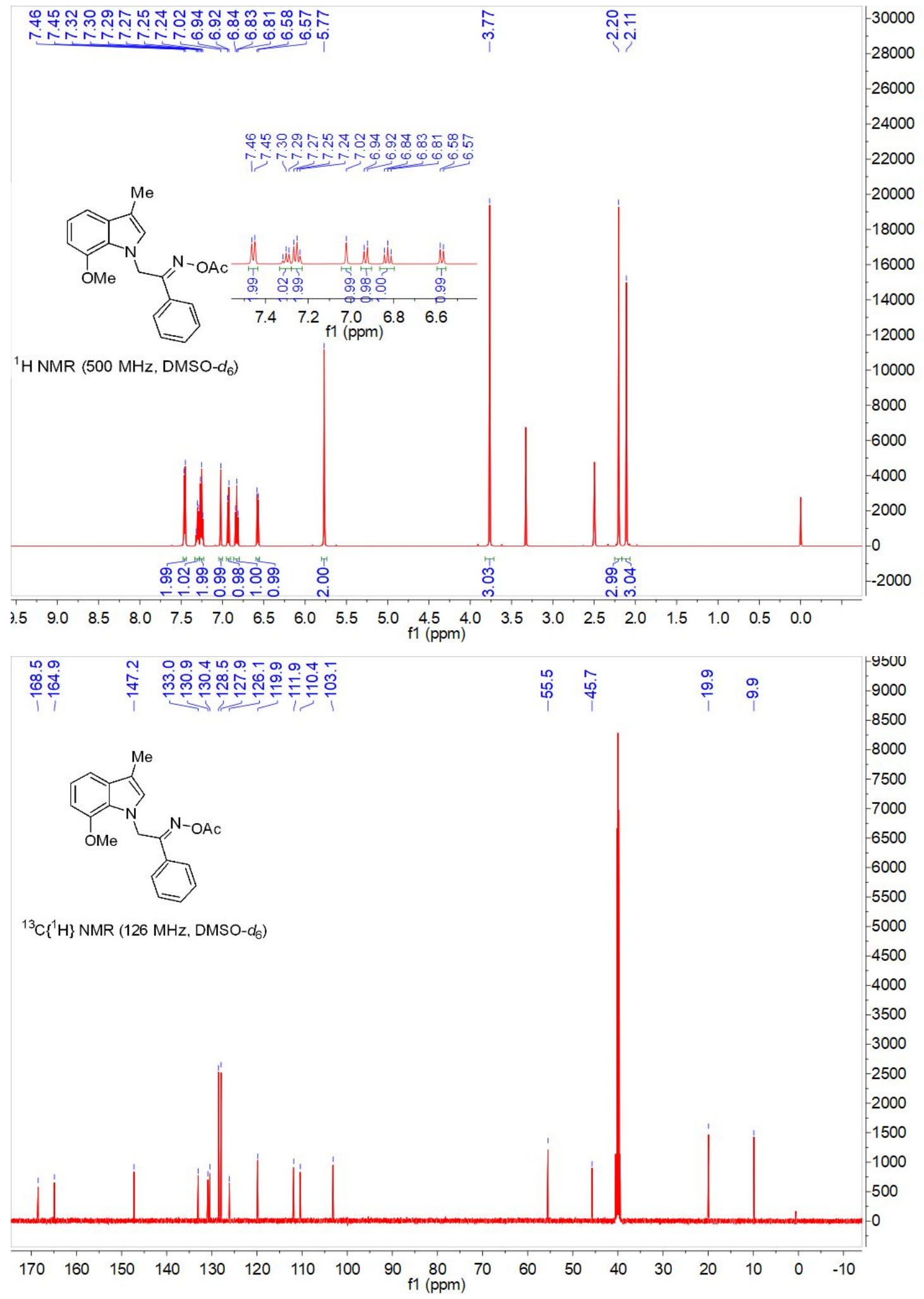


(E)-2-(4-fluoro-3-methyl-1H-indol-1-yl)-1-phenylethan-1-one

O-acetyl

oxime

(1j):

무워ำ

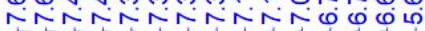

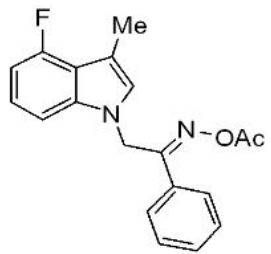

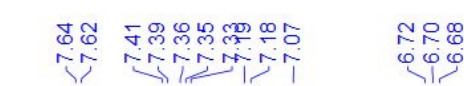

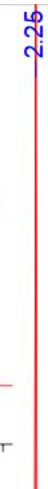

${ }^{1} \mathrm{H}$ NMR $\left(500 \mathrm{MHz}, \mathrm{DMSO}-d_{6}\right)$
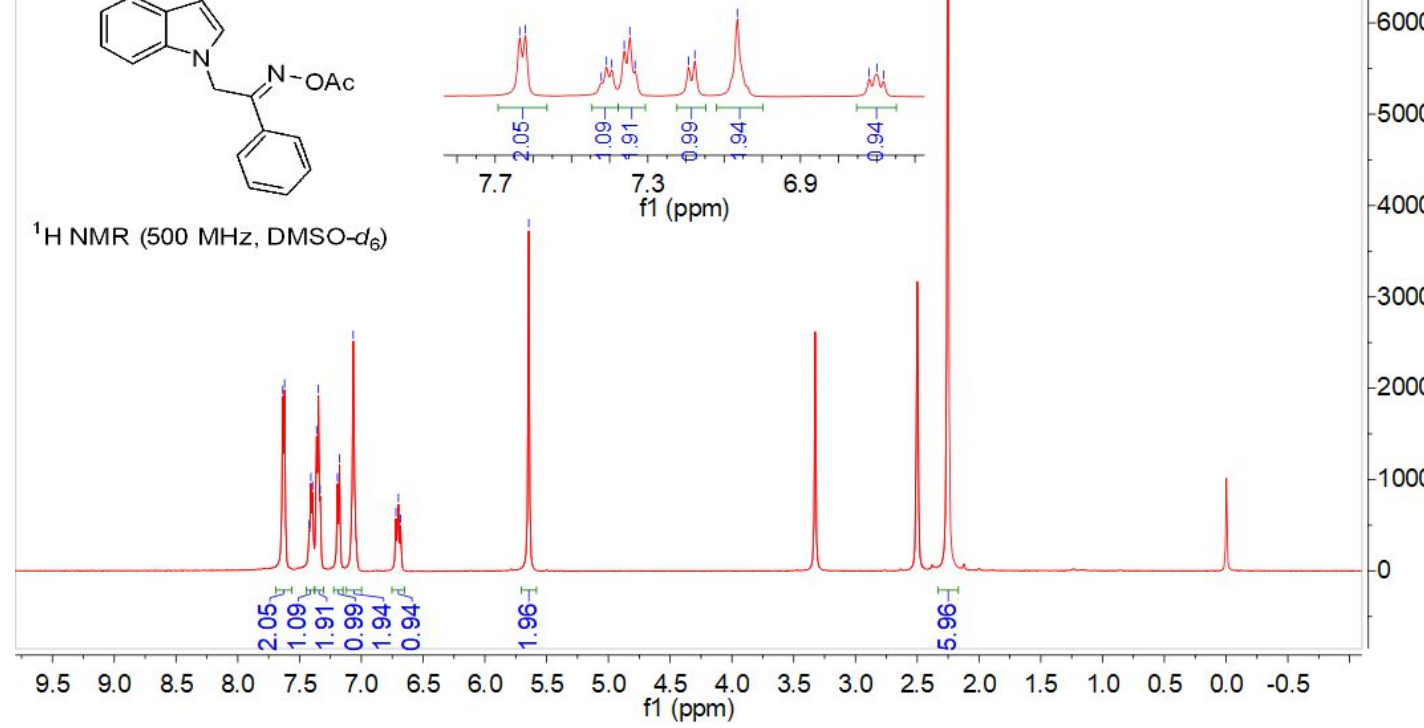

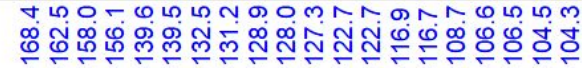
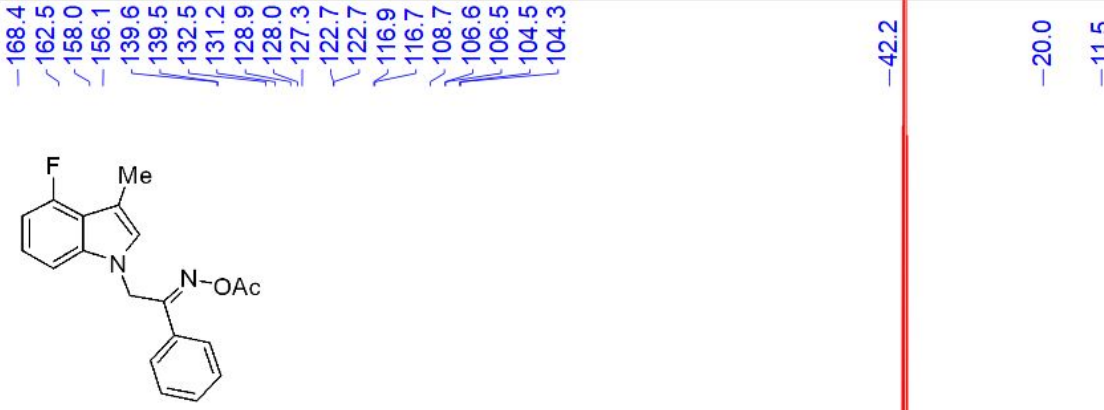

$-5000$

4500

4000

$-3500$

3000

${ }^{13} \mathrm{C}\left\{{ }^{1} \mathrm{H}\right\} \mathrm{NMR}\left(126 \mathrm{MHz}, \mathrm{DMSO}-\mathrm{d}_{6}\right)$

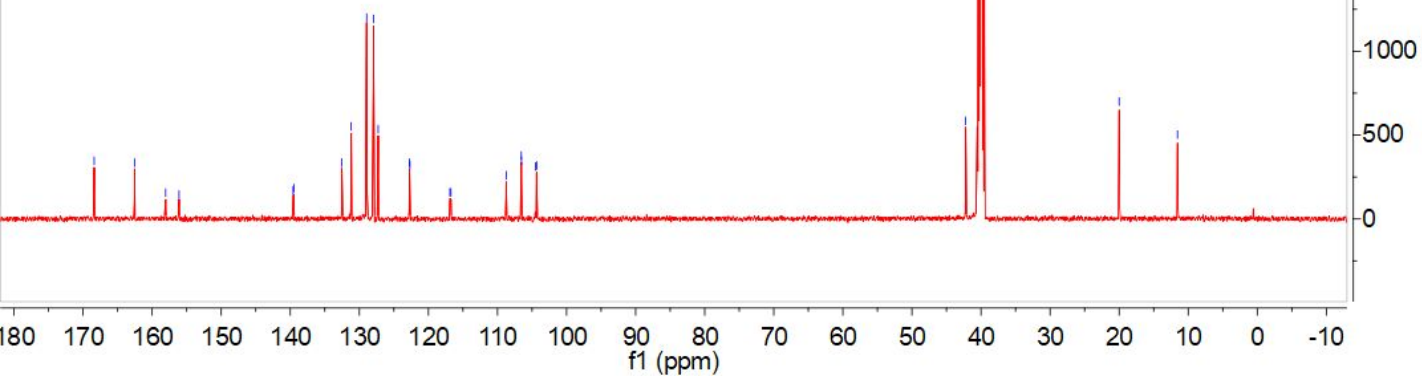


(E)-2-(6-chloro-3-methyl-1H-indol-1-yl)-1-phenylethan-1-one O-acetyl oxime (1k):

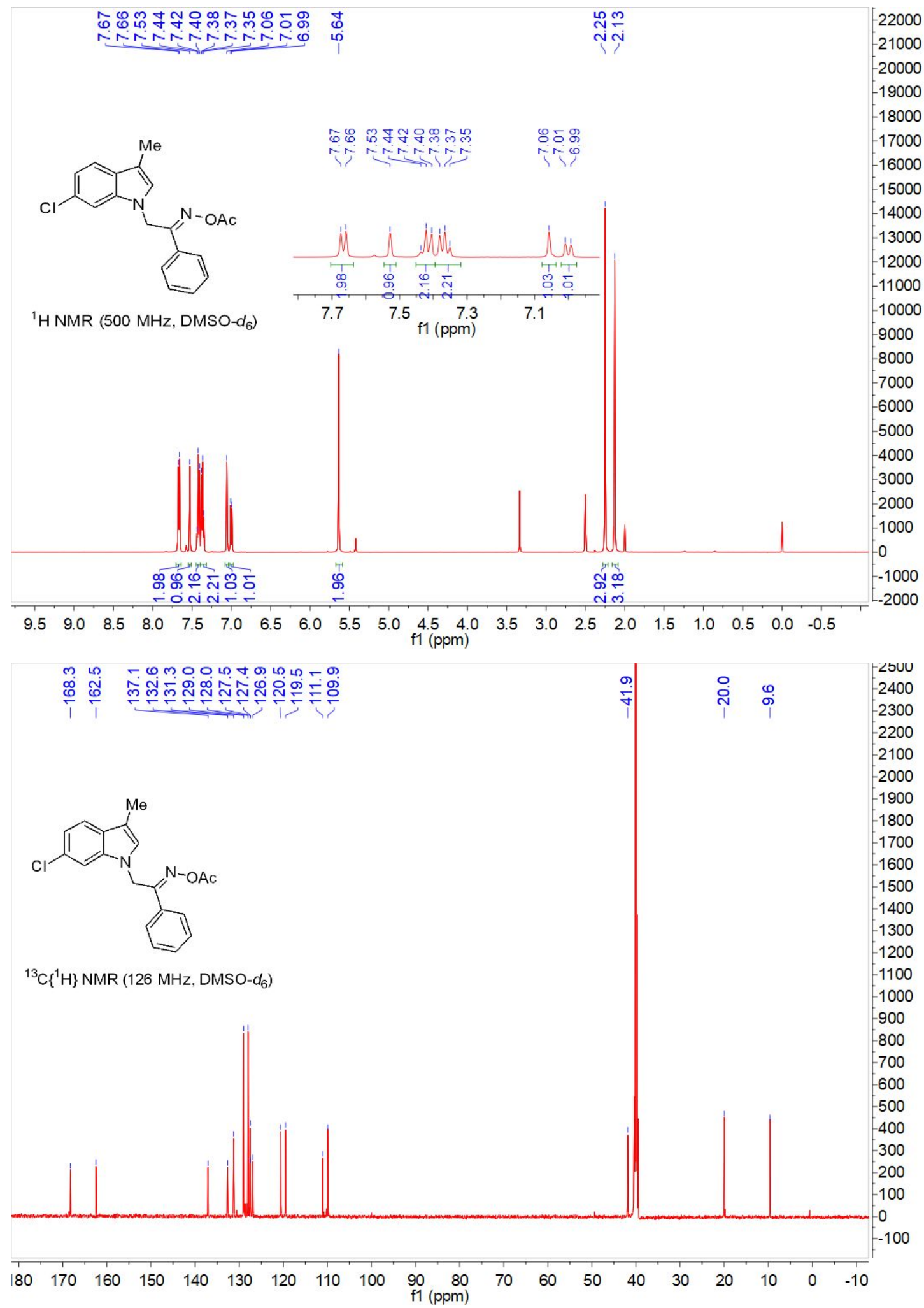


(E)-2-(6-bromo-3-methyl-1H-indol-1-yl)-1-phenylethan-1-one O-acetyl oxime (11):

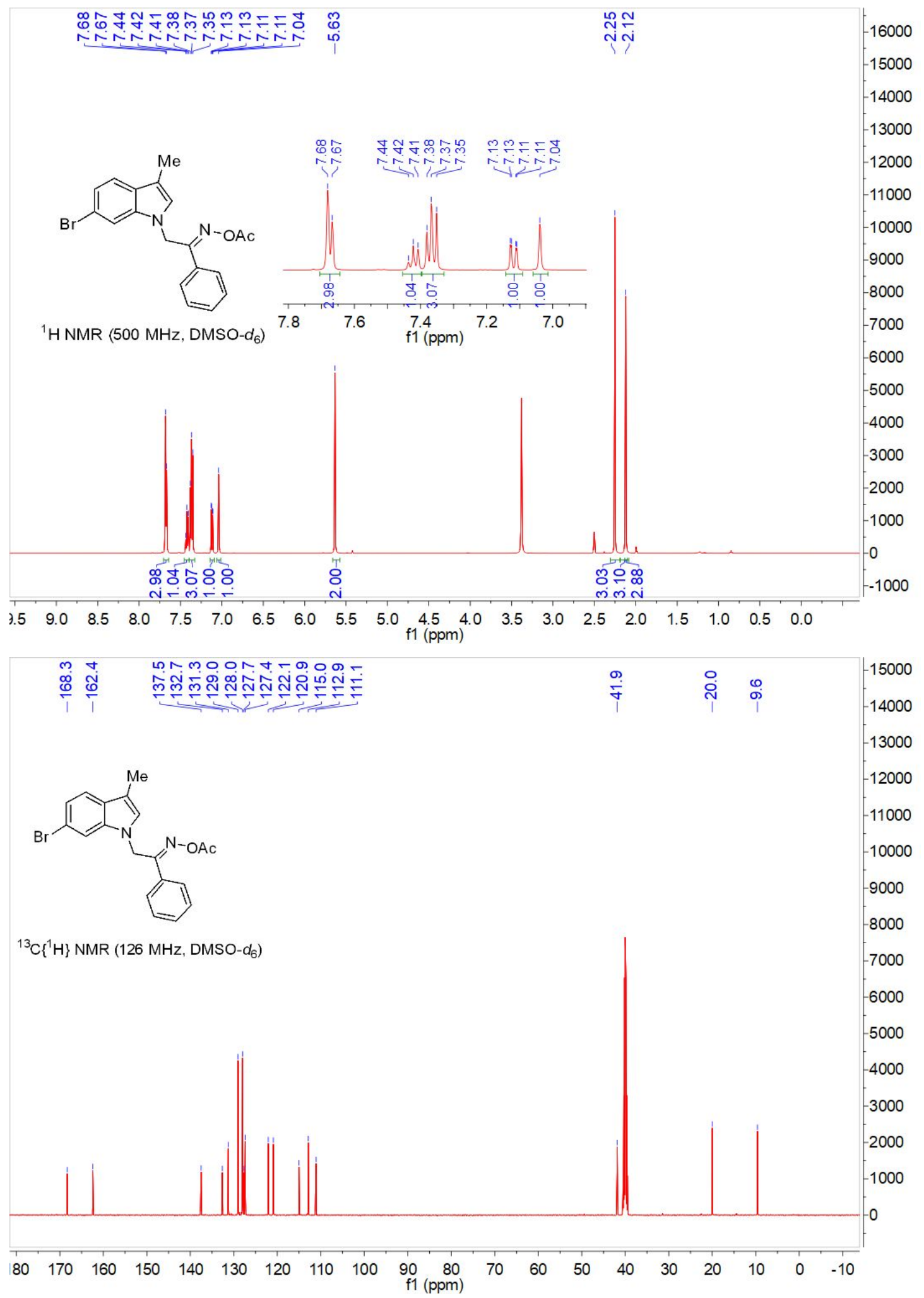


(E)-2-(7-bromo-3-methyl-1H-indol-1-yl)-1-phenylethan-1-one O-acetyl oxime (1m):

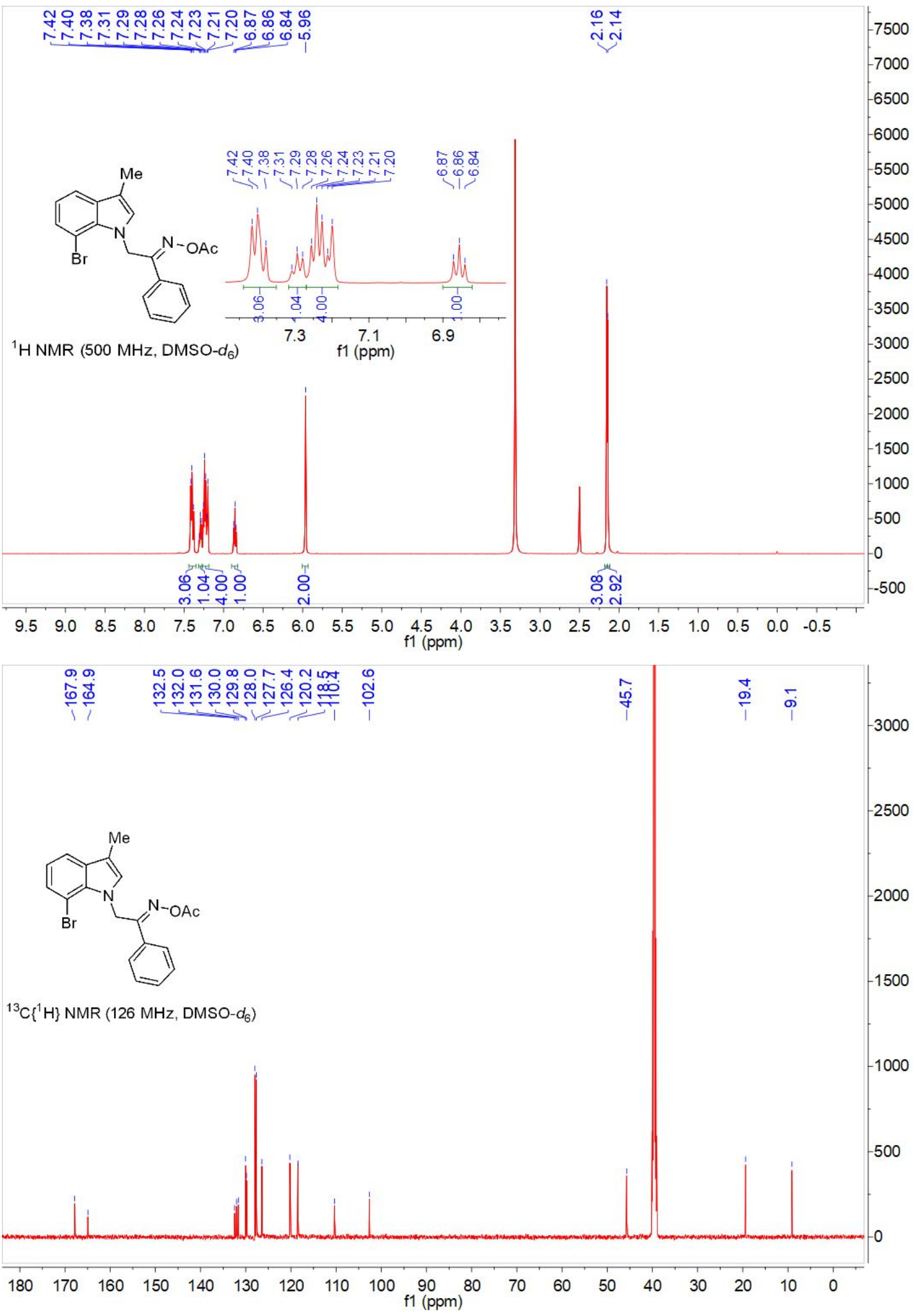


(E)-2-(3-methyl-1H-indol-1-yl)-1-(p-tolyl)ethan-1-one O-acetyl oxime (1n):

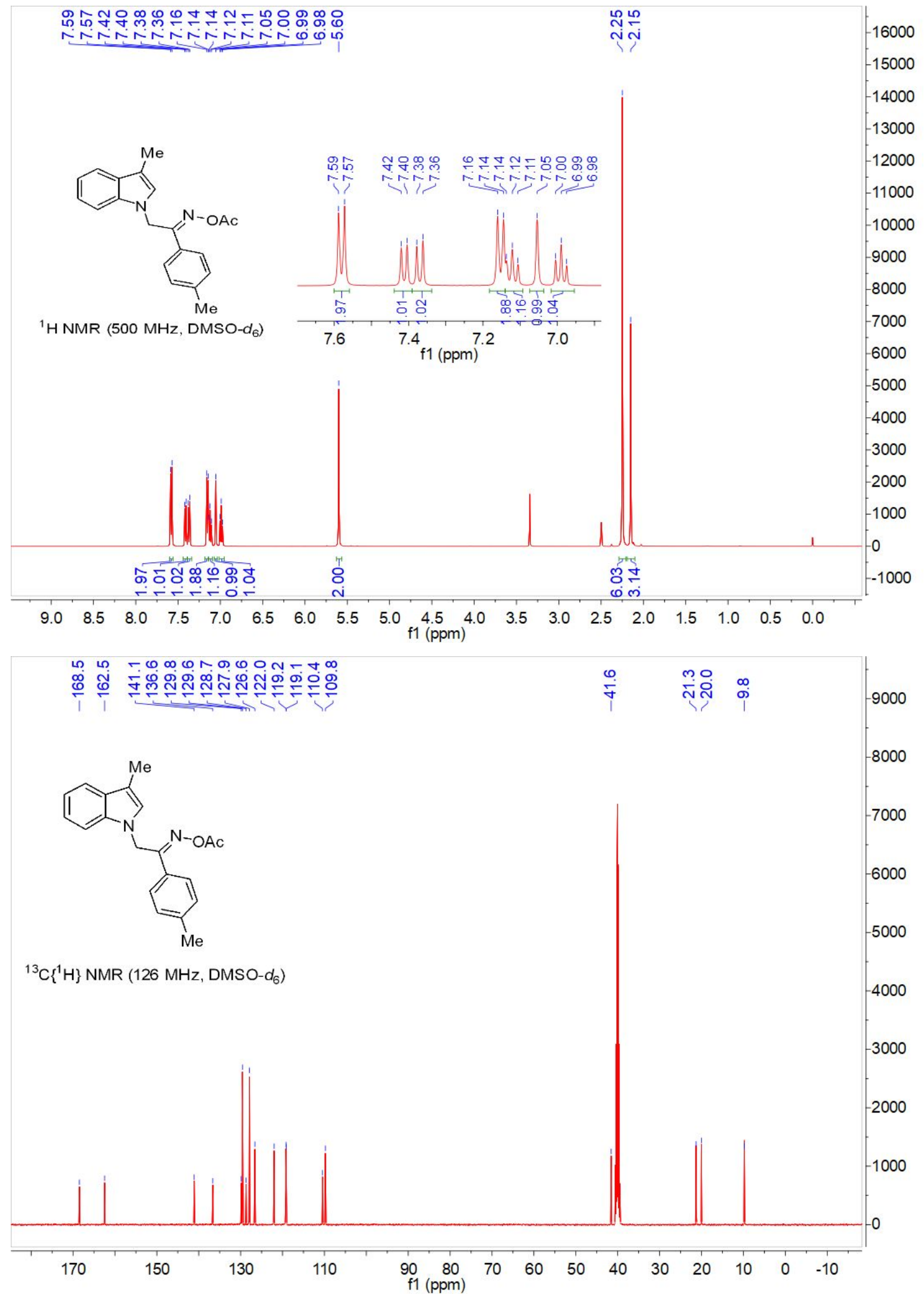


(E)-1-(3-methoxyphenyl)-2-(3-methyl-1H-indol-1-yl)ethan-1-one O-acetyl oxime (10):

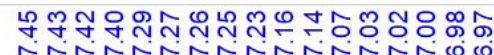

NNNNNNNNNNTOO

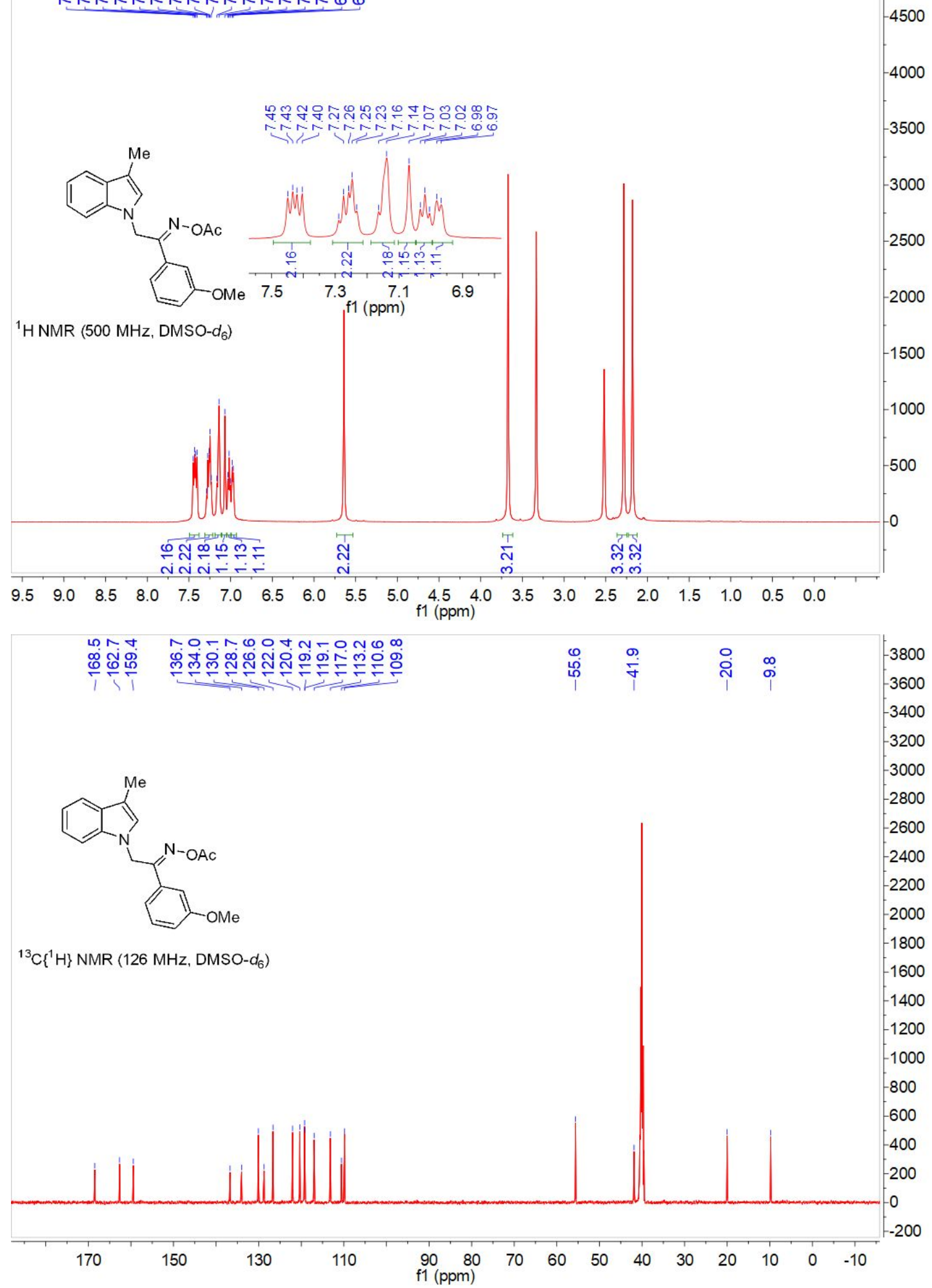


(E)-1-(4-methoxyphenyl)-2-(3-methyl-1H-indol-1-yl)ethan-1-one O-acetyl oxime (1p):

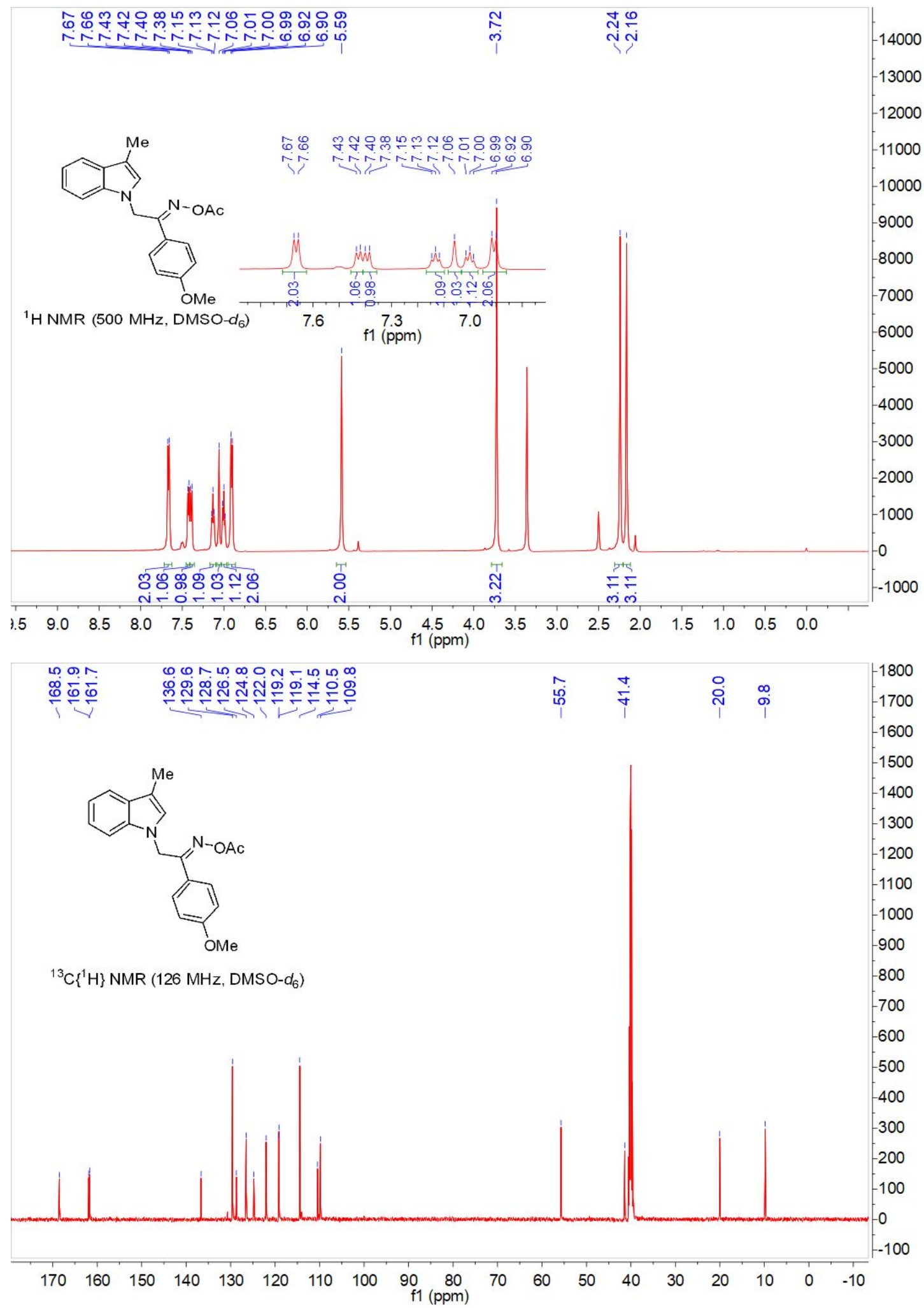


(E)-1-(2-fluorophenyl)-2-(3-methyl-1H-indol-1-yl)ethan-1-one

(1q):

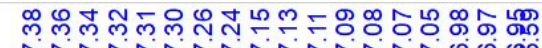

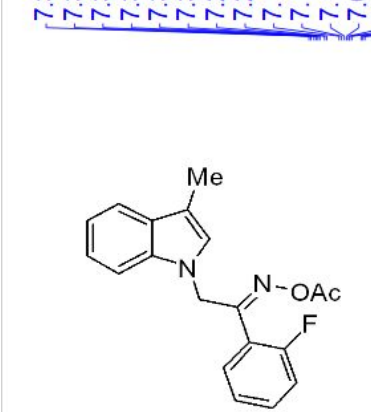

${ }^{1} \mathrm{H}$ NMR $\left(500 \mathrm{MHz}, \mathrm{DMSO}-d_{6}\right)$

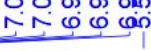

5000

4500

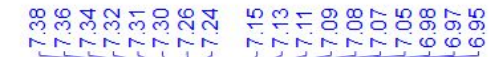

$-4000$

3500

$-3000$

2500

2000

1500

1000

500

กับขึั

8

mำ

$\begin{array}{lllllllllllllllllllll}9.5 & 9.0 & 8.5 & 8.0 & 7.5 & 7.0 & 6.5 & 6.0 & 5.5 & 5.0 & \begin{array}{l}4.5 \\ \mathrm{f} 1(\mathrm{ppm})\end{array} & 4.0 & 3.5 & 3.0 & 2.5 & 2.0 & 1.5 & 1.0 & 0.5 & 0.0\end{array}$

กุ

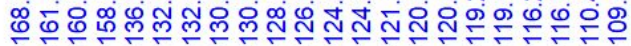

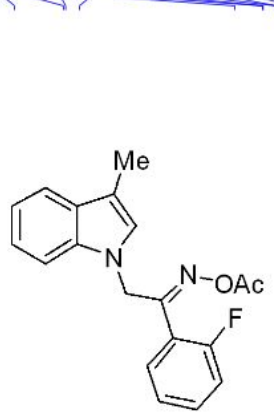

${ }^{13} \mathrm{C}\left\{{ }^{1} \mathrm{H}\right\}$ NMR $\left(126 \mathrm{MHz}, \mathrm{DMSO}-d_{6}\right)$

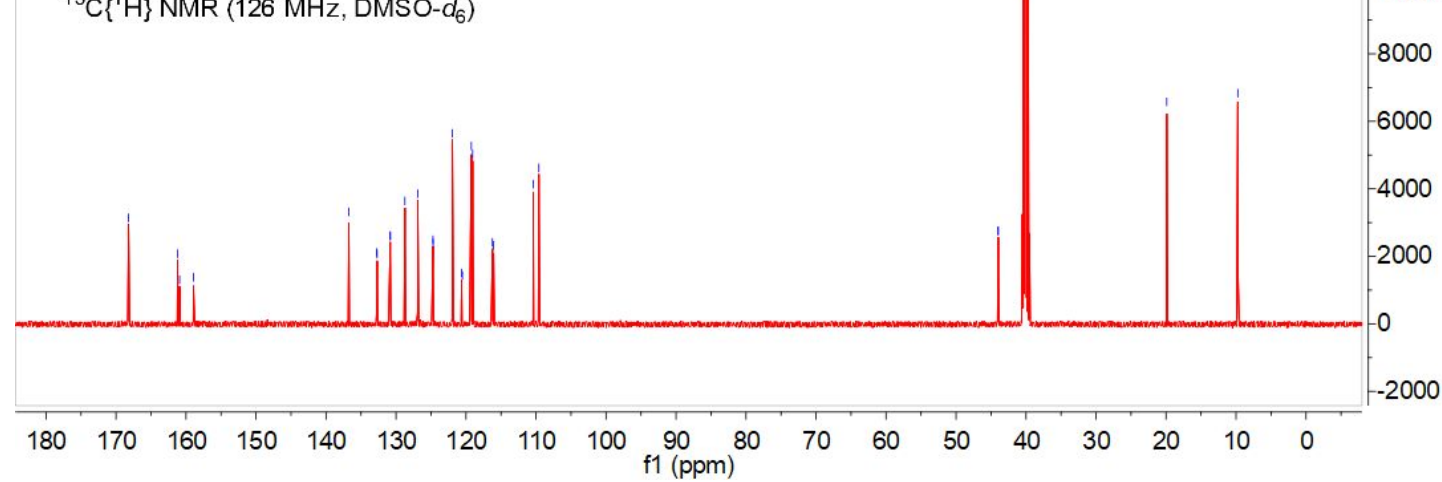


(E)-1-(3-fluorophenyl)-2-(3-methyl-1H-indol-1-yl)ethan-1-one O-acetyl oxime (1r):

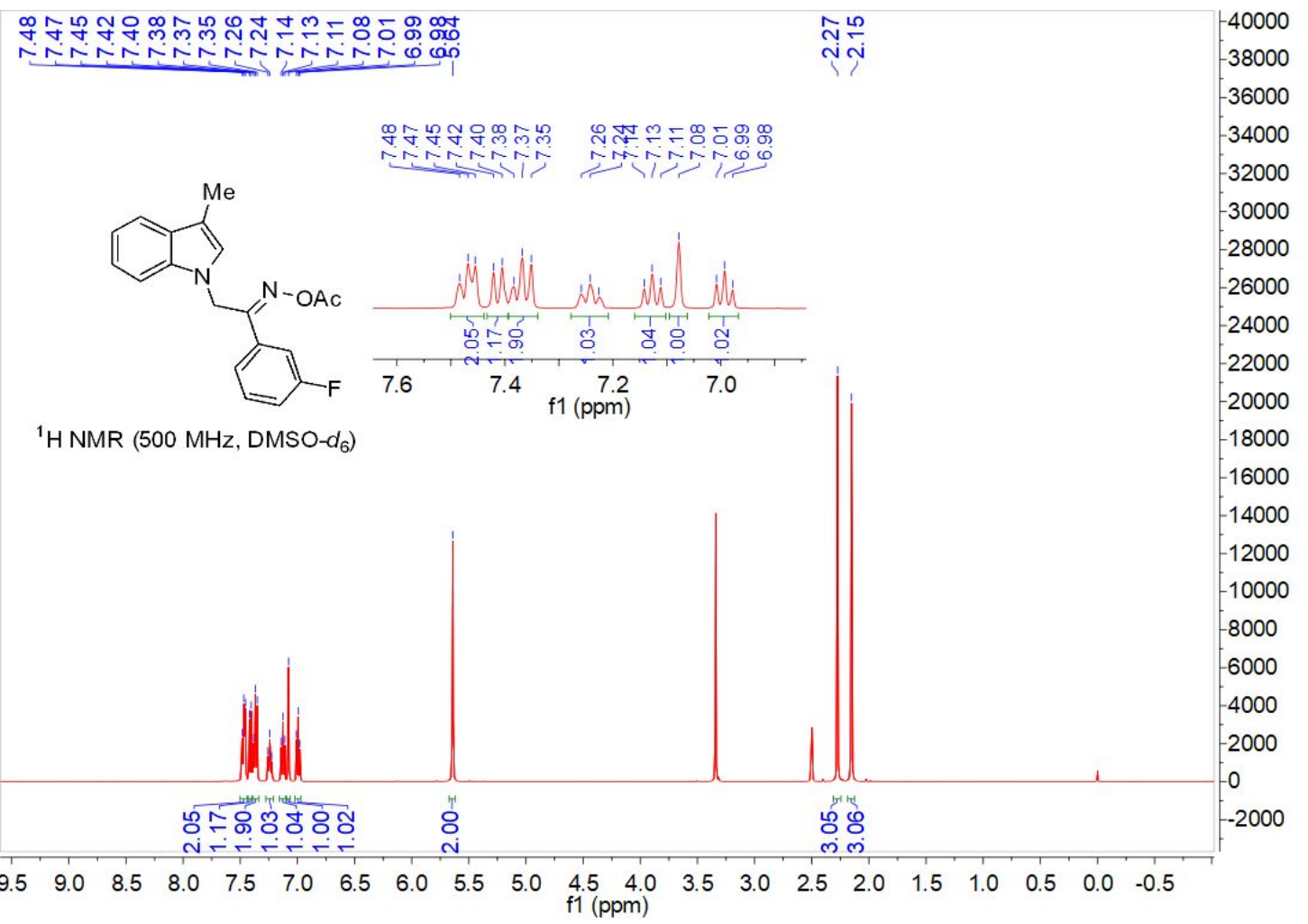

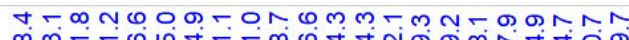

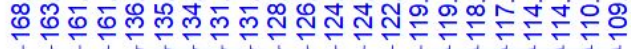

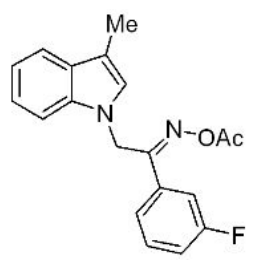

${ }^{13} \mathrm{C}\left\{{ }^{1} \mathrm{H}\right\}$ NMR (126 MHz, DMSO-d $)$

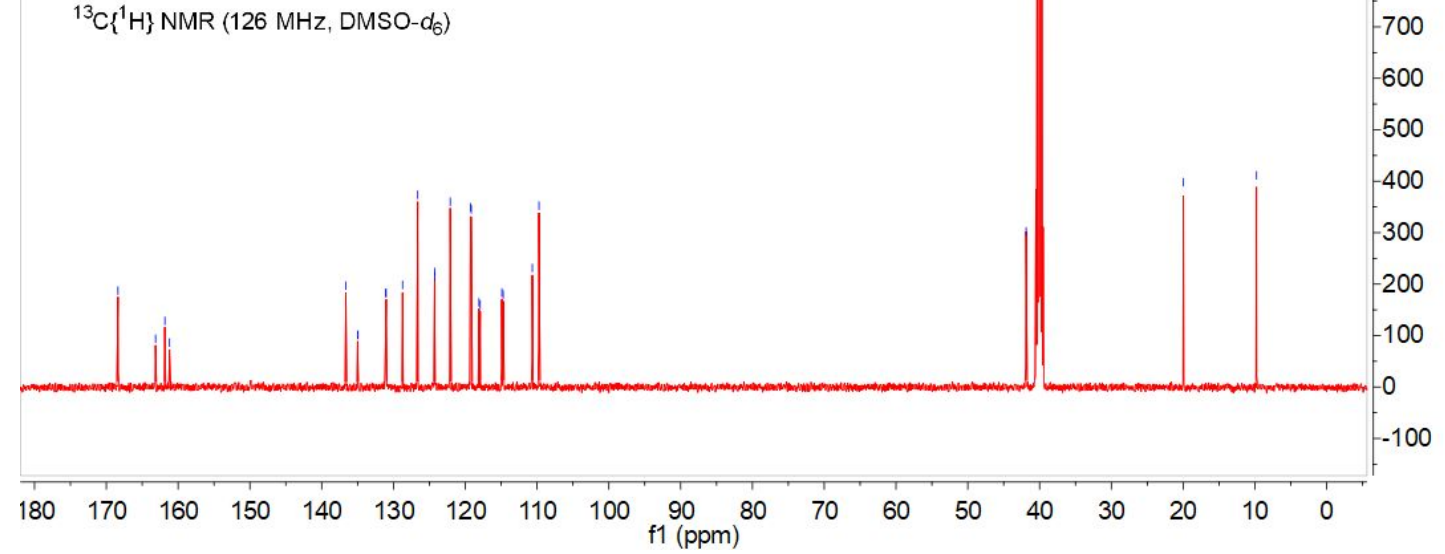


(E)-1-(4-fluorophenyl)-2-(3-methyl-1H-indol-1-yl)ethan-1-one

O-acetyl oxime

(1s):

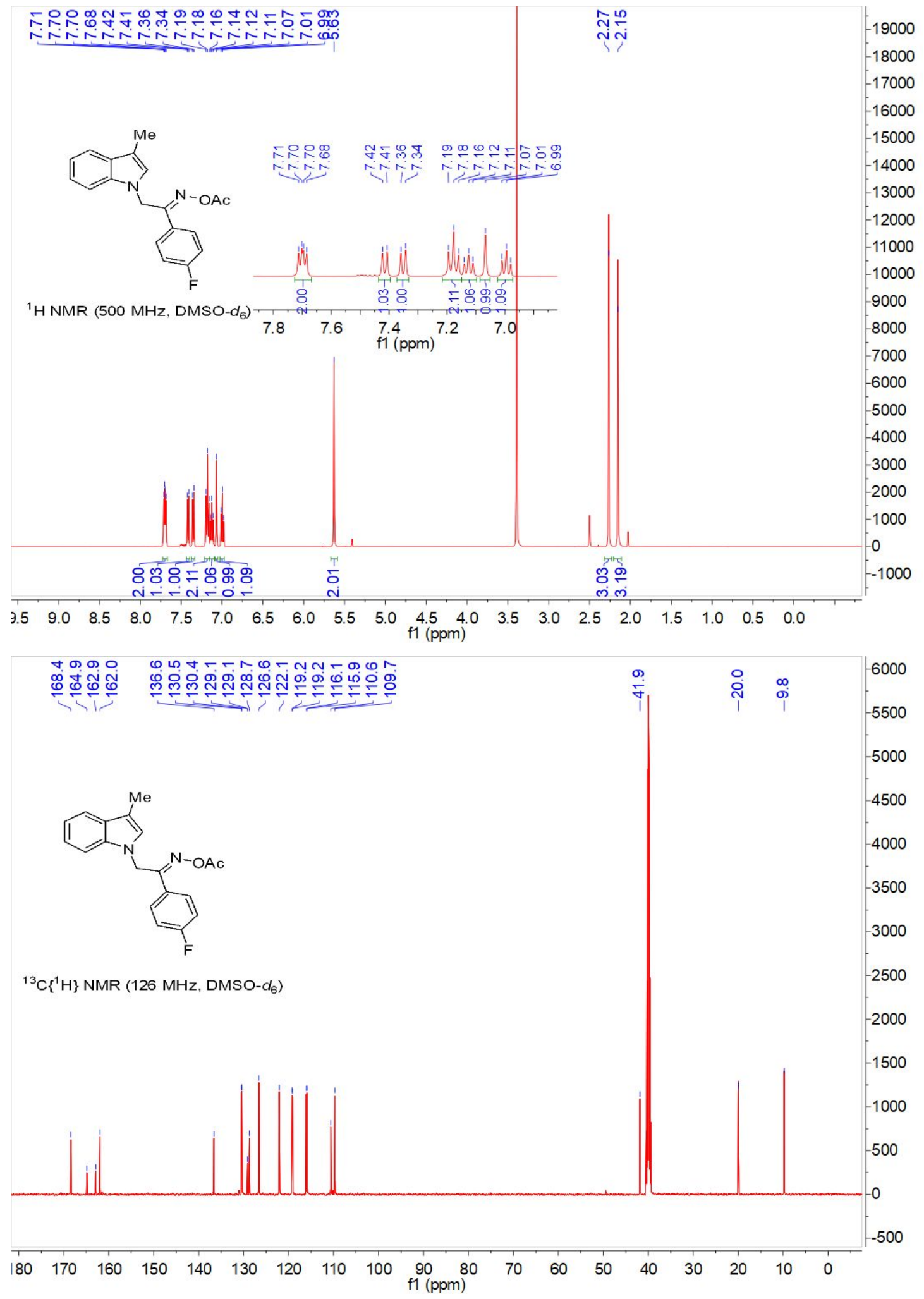


(E)-1-(2,4-difluorophenyl)-2-(3-methyl-1H-indol-1-yl)ethan-1-one

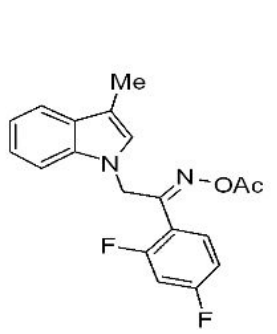

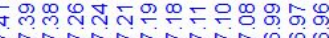

${ }^{1} \mathrm{H}$ NMR $\left(500 \mathrm{MHz}, \mathrm{DMSO}-d_{6}\right)$
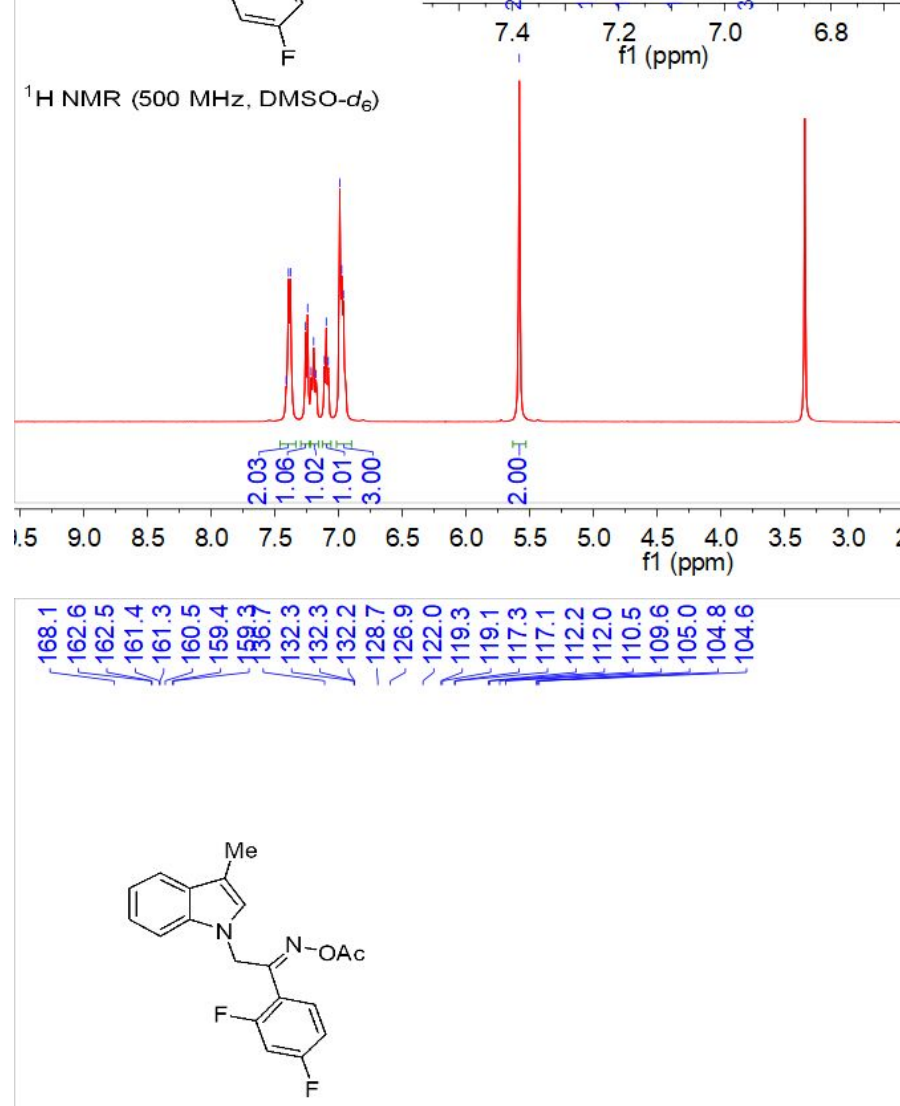

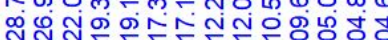

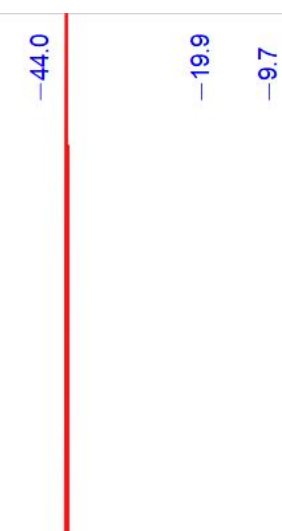

$-4500$

${ }^{13} \mathrm{C}\left\{{ }^{1} \mathrm{H}\right\}$ NMR (126 MHz, DMSO- $\left.d_{6}\right)$

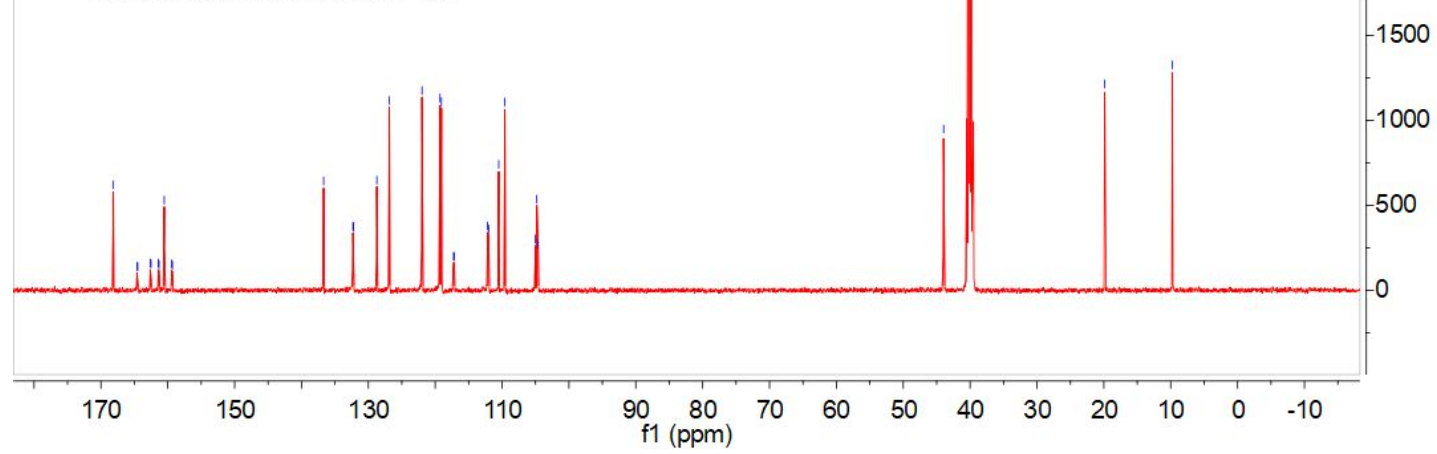


(E)-1-(2-chlorophenyl)-2-(3-methyl-1H-indol-1-yl)ethan-1-one O-acetyl oxime(1u):

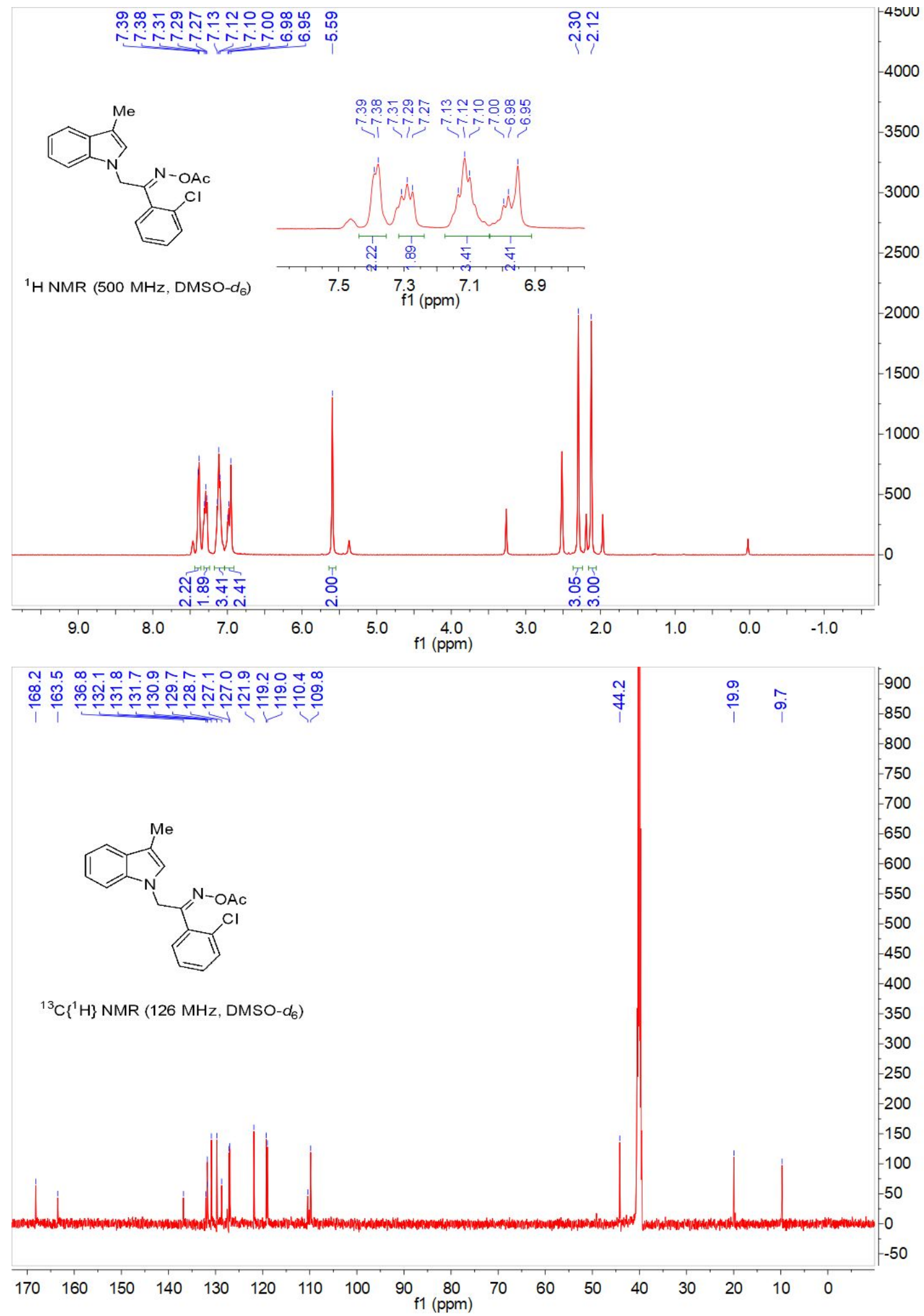


(E)-1-(3-chlorophenyl)-2-(3-methyl-1H-indol-1-yl)ethan-1-one O-acetyl oxime (1v):

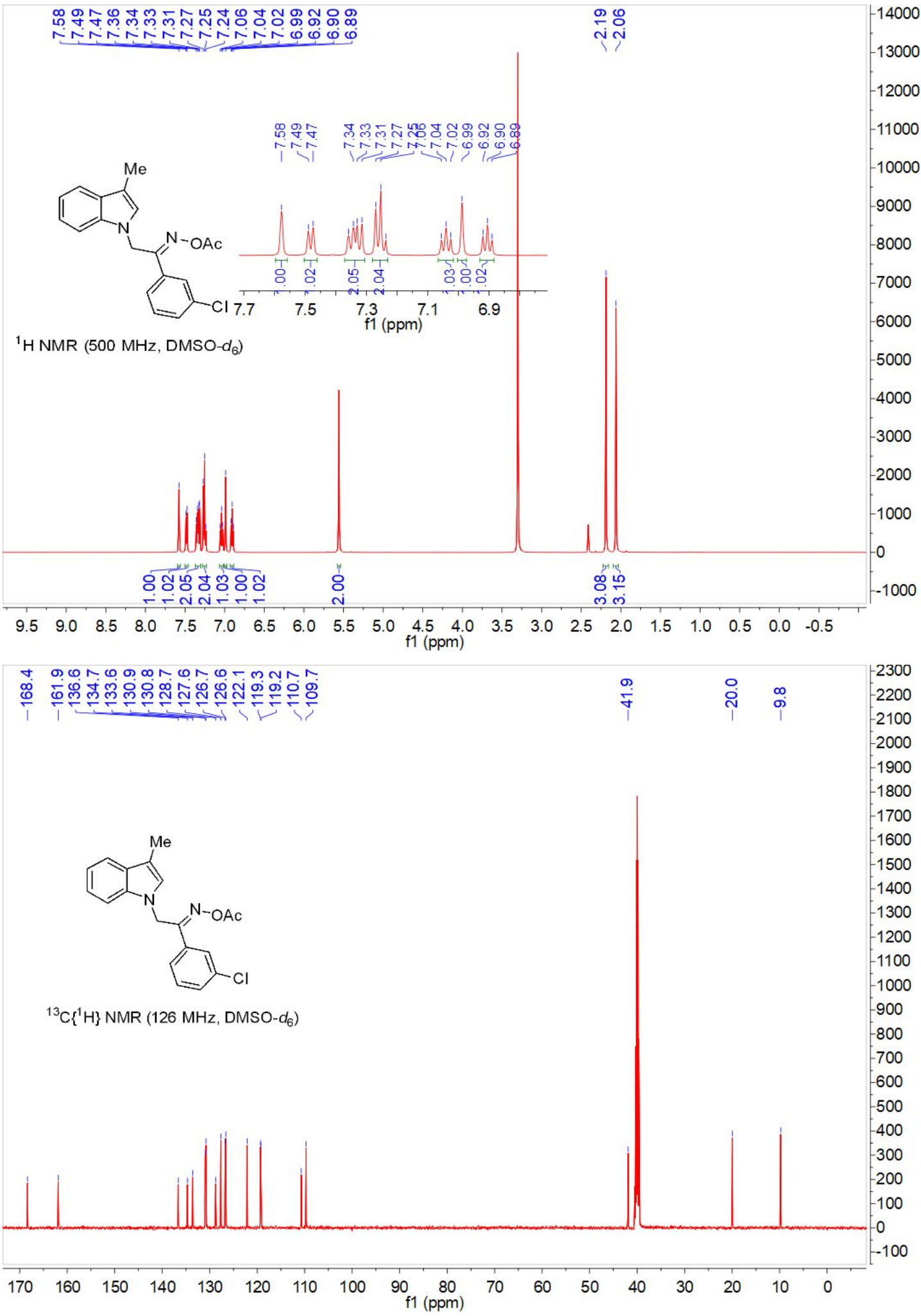


(E)-1-(4-chlorophenyl)-2-(3-methyl-1H-indol-1-yl)ethan-1-one O-acetyl oxime (1w):

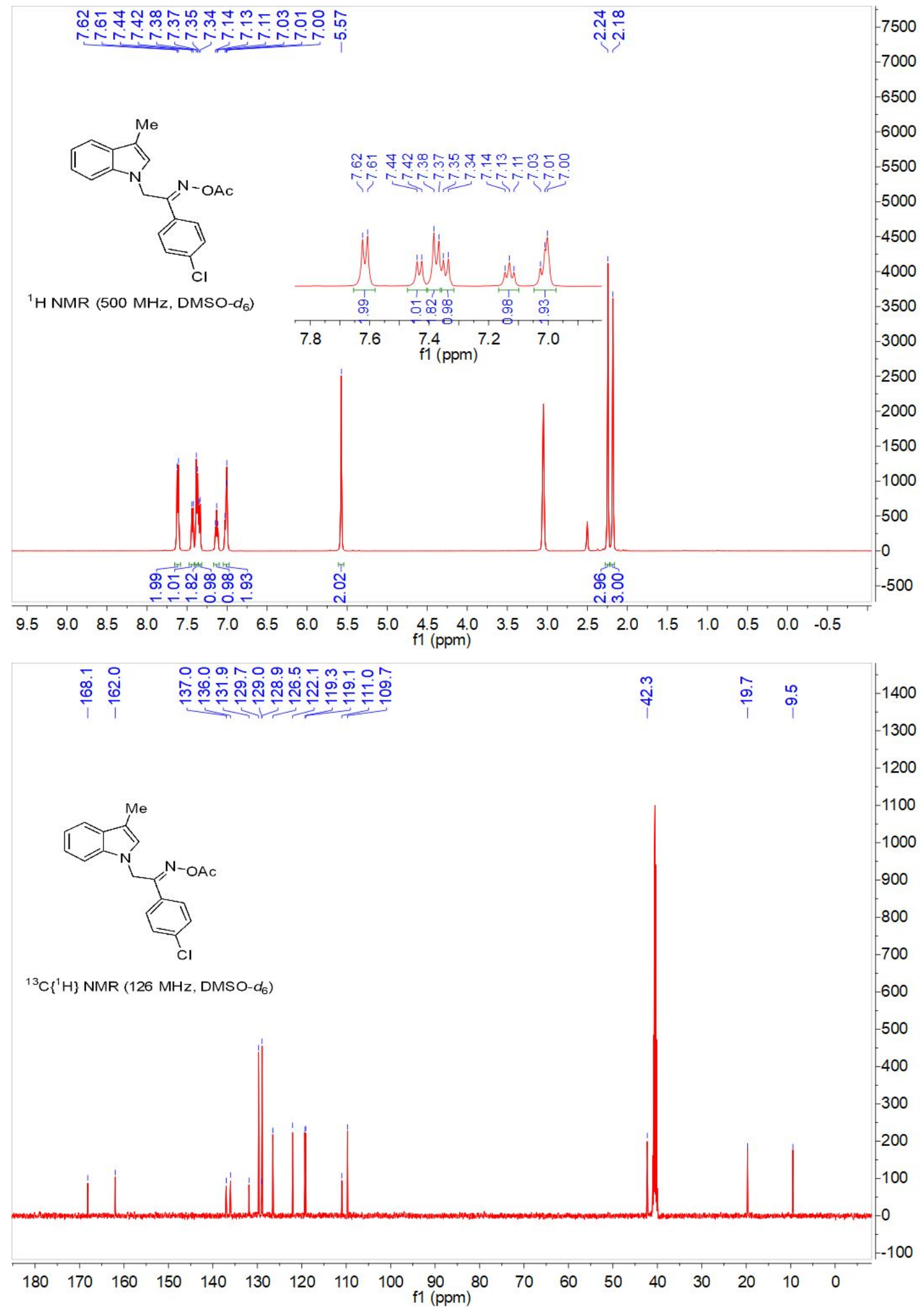


(E)-1-(3,4-dichlorophenyl)-2-(3-methyl-1H-indol-1-yl)ethan-1-one O-acetyl oxime(1x):

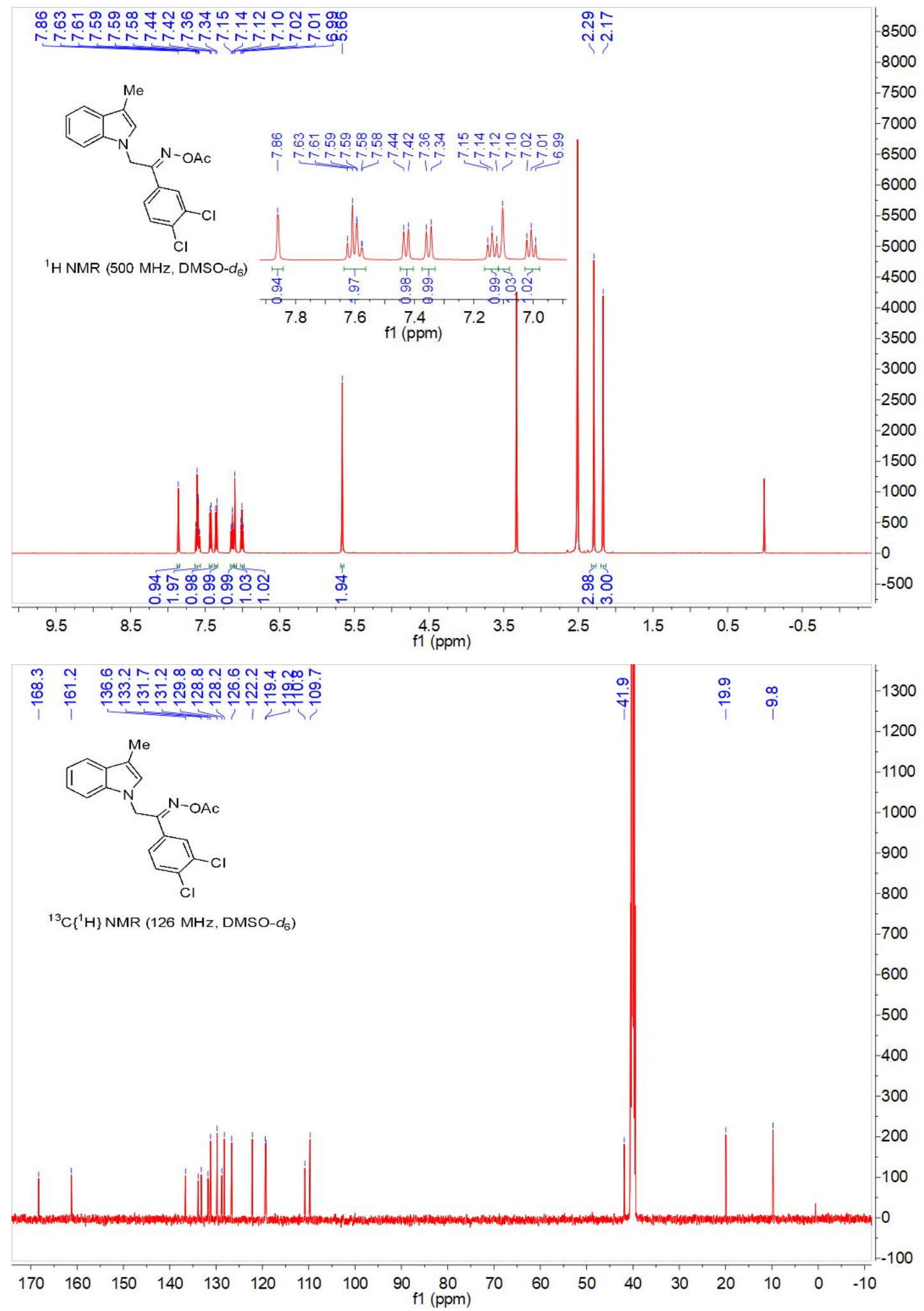


(E)-1-(4-bromophenyl)-2-(3-methyl-1H-indol-1-yl)ethan-1-one O-acetyl oxime (1y):

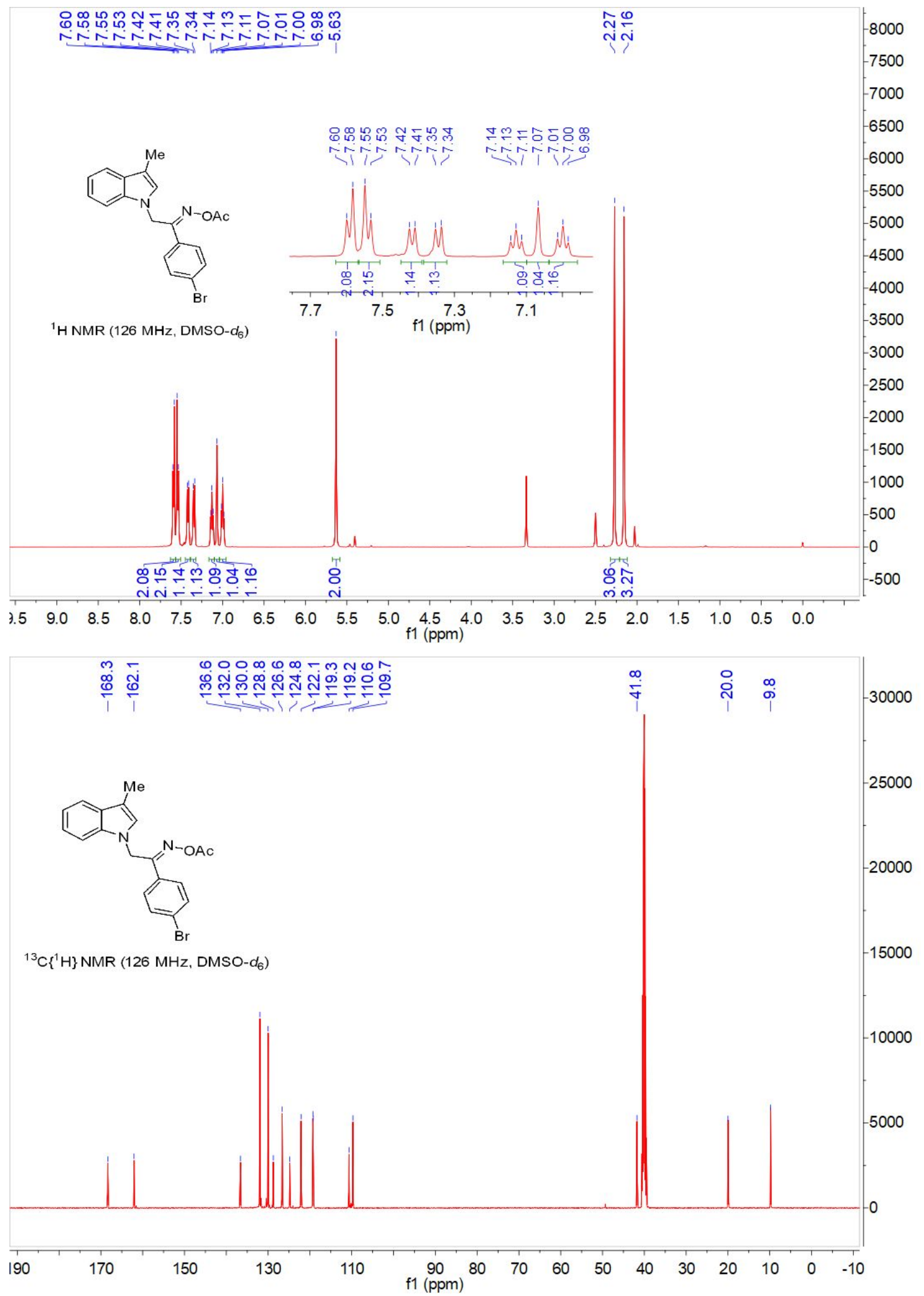


9,9'-dimethyl-2,2'-diphenyl-9H,9'H-9,9'-biimidazo[1,2-a]indole (dl-2a):

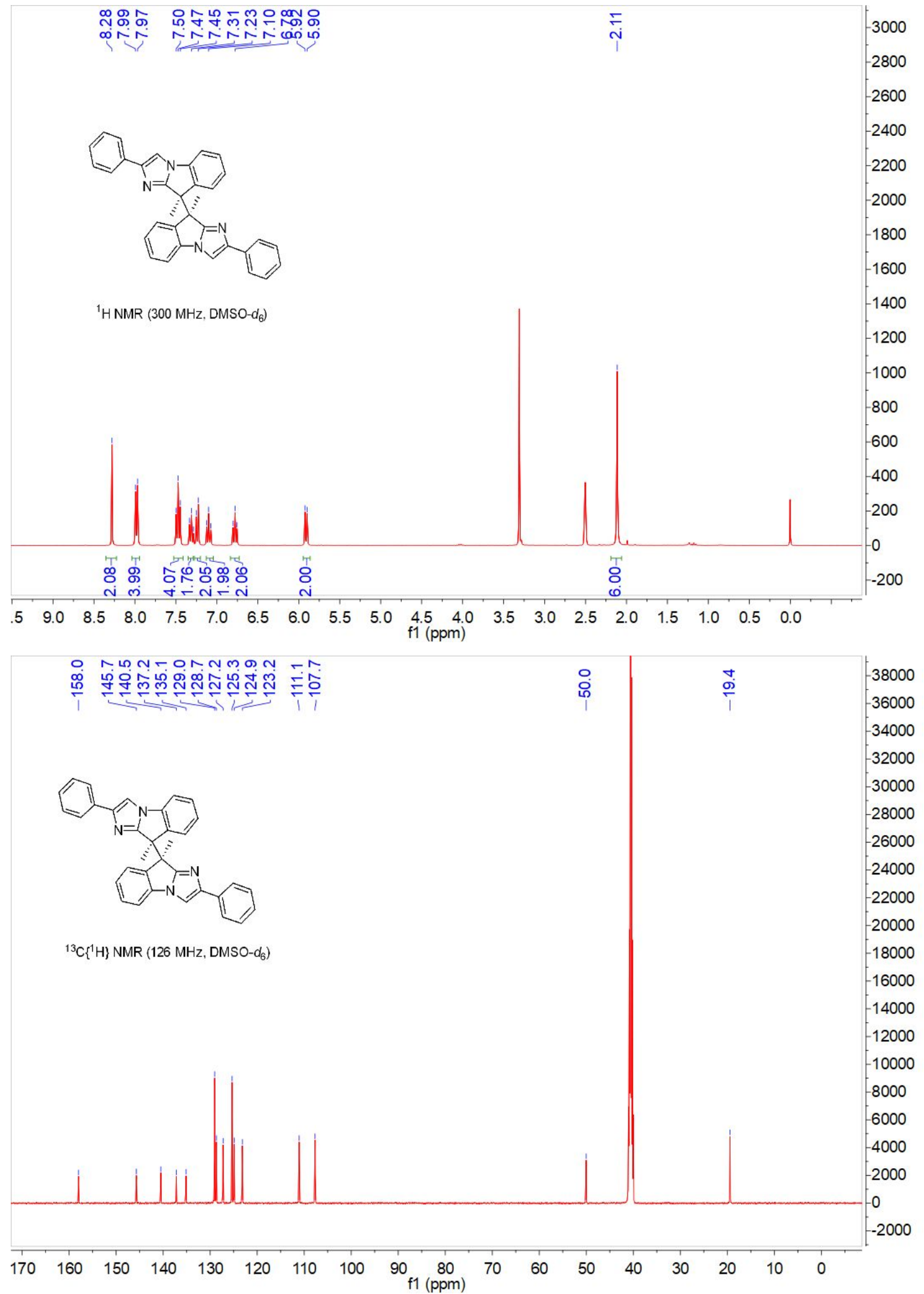


9,9'-dimethyl-2,2'-diphenyl-9H,9'H-9,9'-biimidazo[1,2-a]indole (meso-2a):

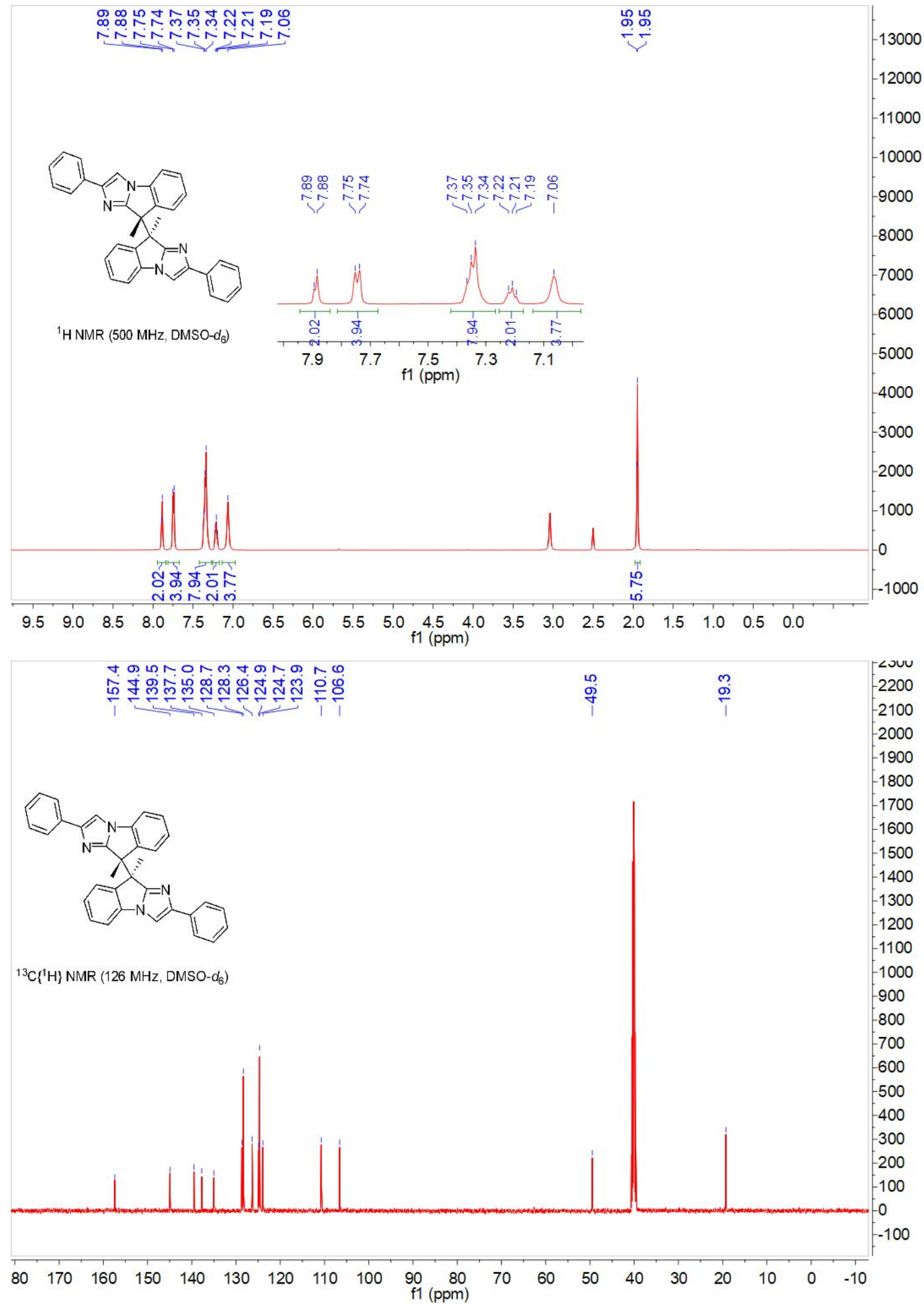


9,9'-diethyl-2,2'-diphenyl-9H,9'H-9,9'-biimidazo[1,2-a]indole (dl-2b):

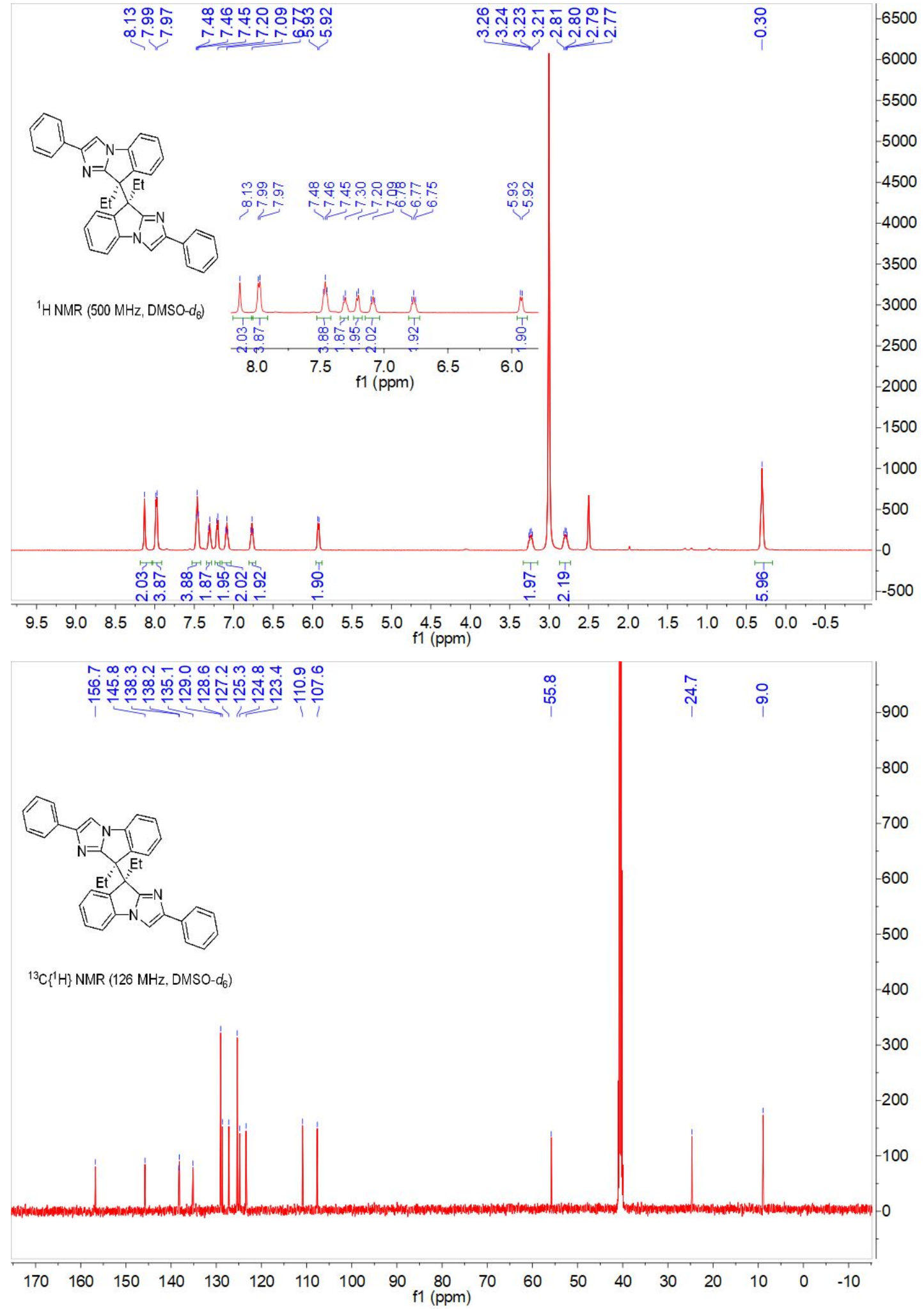


9,9'-diethyl-2,2'-diphenyl-9H,9'H-9,9'-biimidazo[1,2-a]indole (meso-2b):

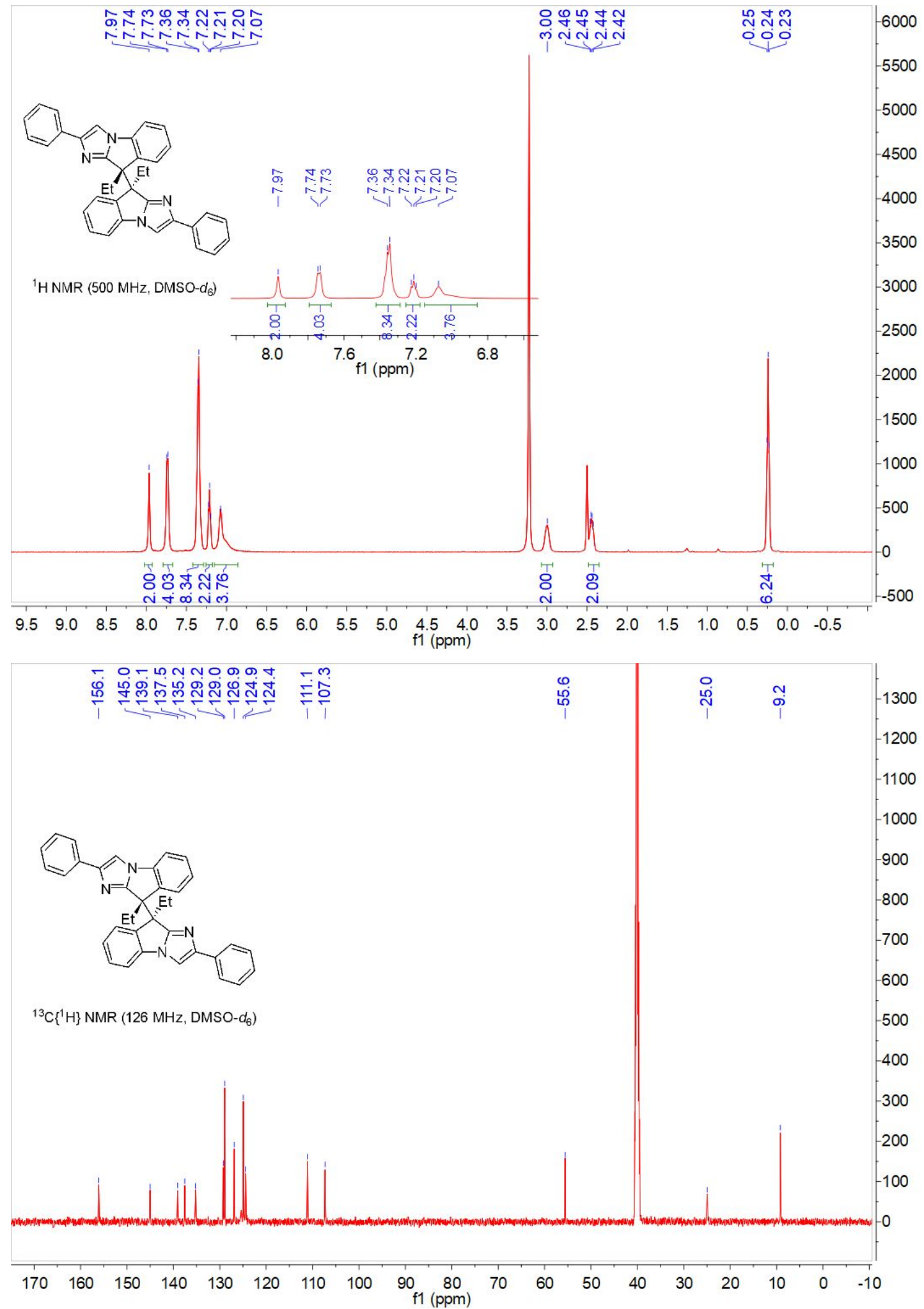

9,9'-diisopropyl-2,2'-diphenyl-9H,9'H-9,9'-biimidazo[1,2-a]indole (meso-2c): 

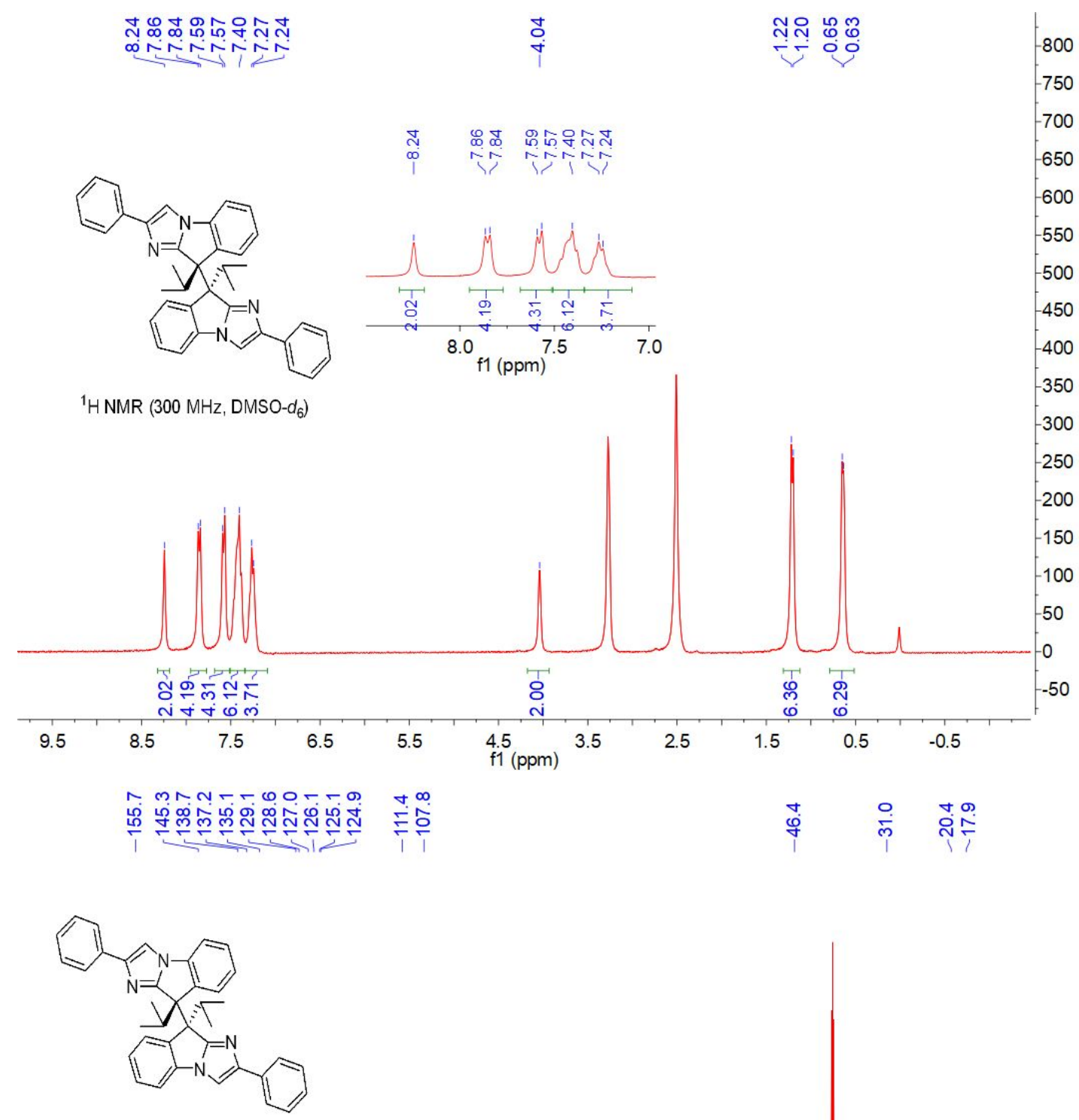

${ }^{13} \mathrm{C}\left\{{ }^{1} \mathrm{H}\right\}$ NMR (126 MHz, DMSO-d $\left.d_{6}\right)$
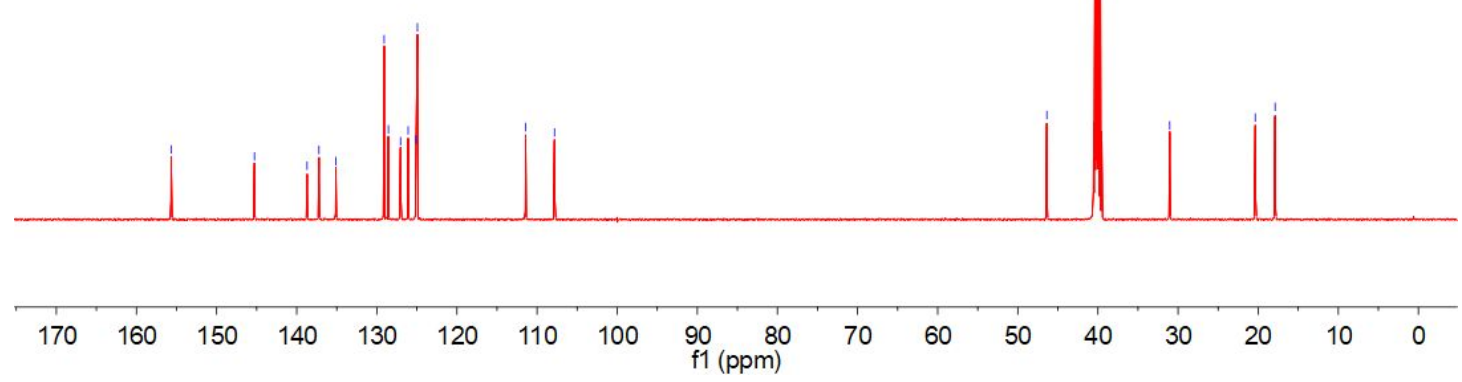

9,9'-diisobutyl-2,2'-diphenyl-9H,9'H-9,9'-biimidazo[1,2-a]indole (dl-2d): 


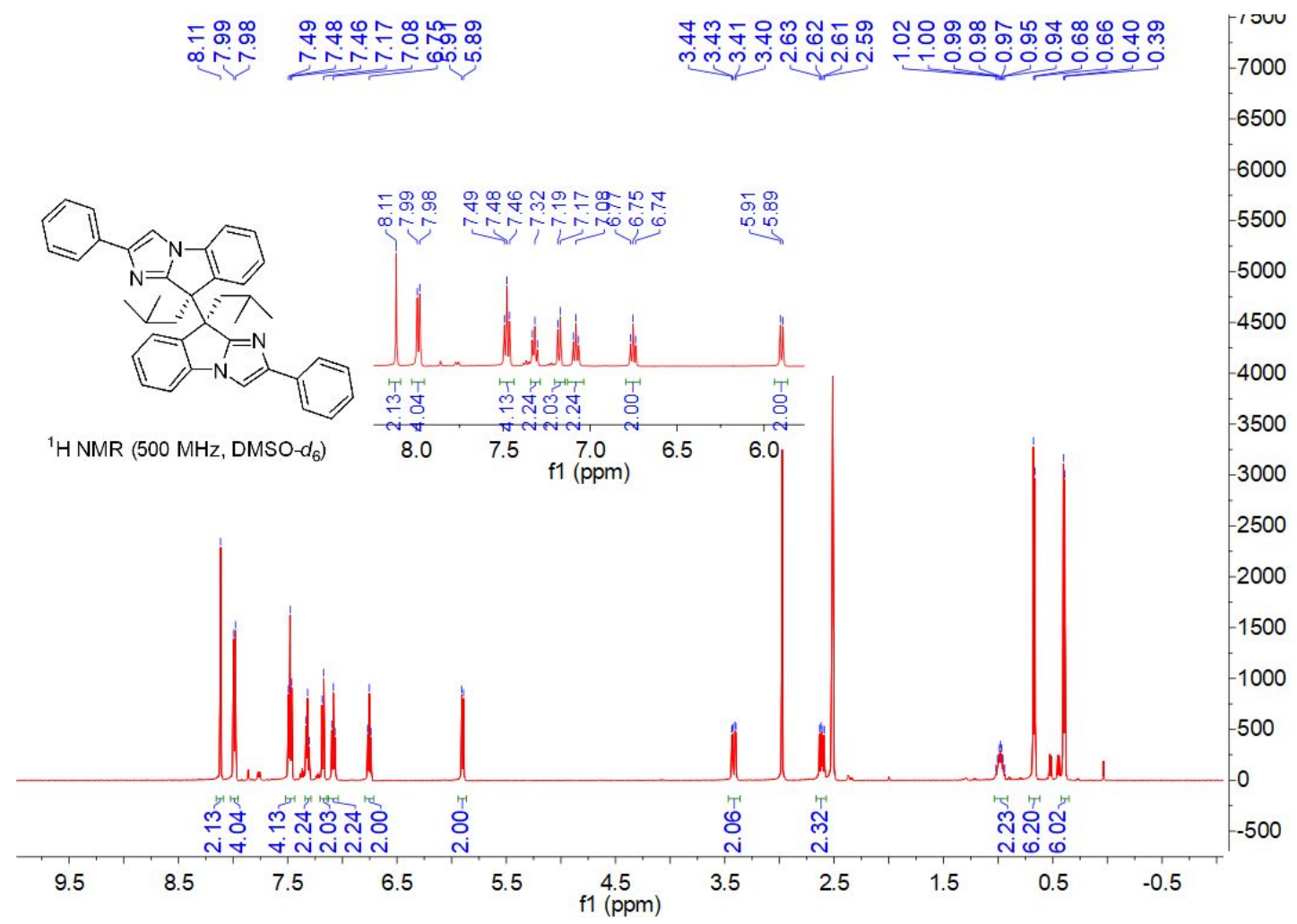

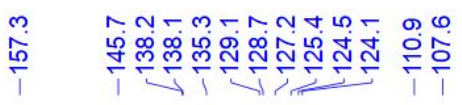

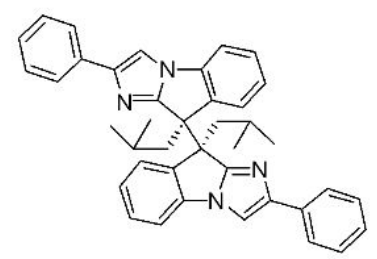

${ }^{13} \mathrm{C}\left\{{ }^{1} \mathrm{H}\right\}$ NMR $\left(126 \mathrm{MHz}, \mathrm{DMSO}-d_{6}\right)$
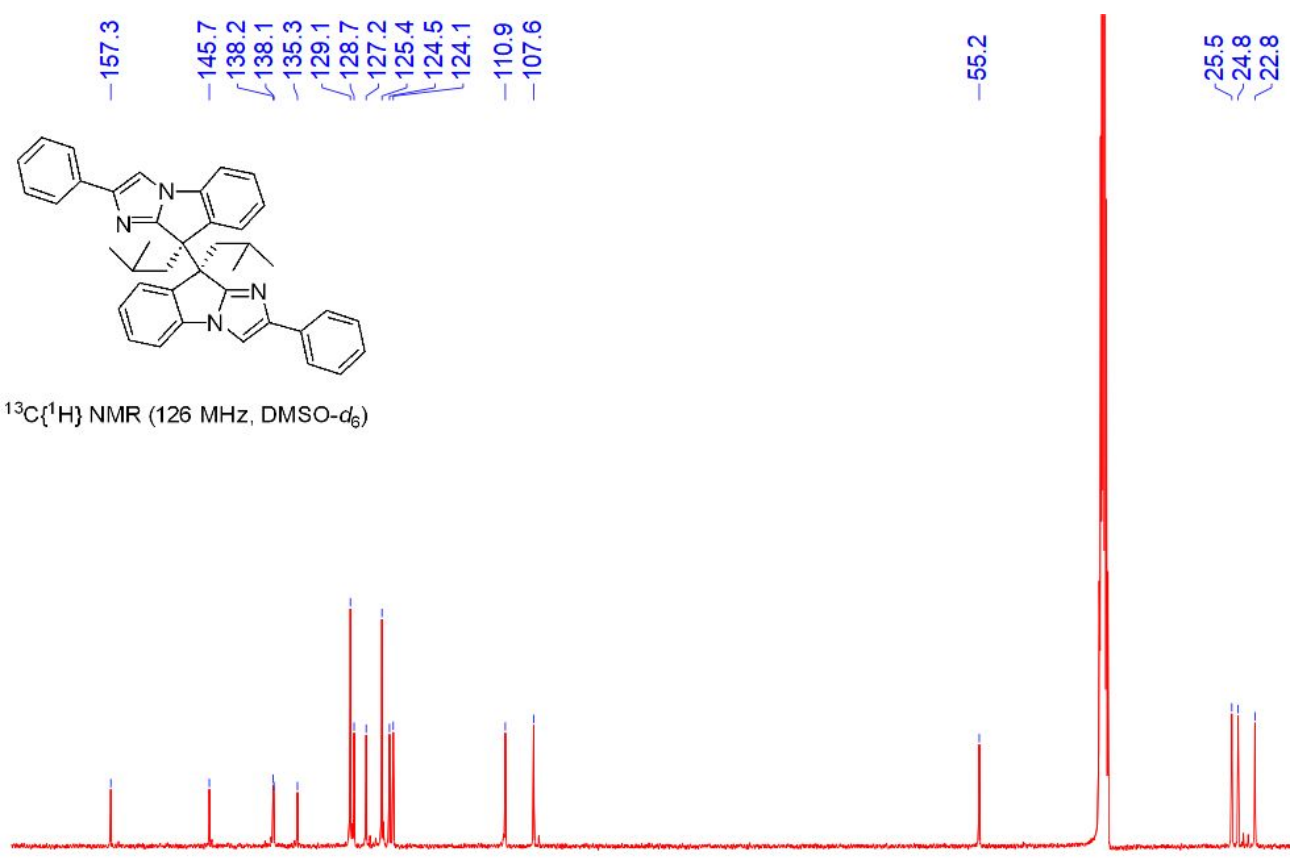

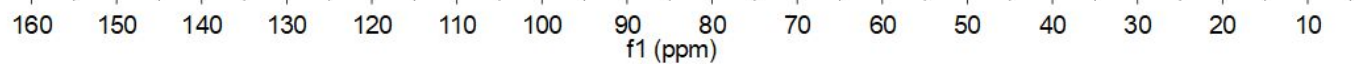


7,7',9,9'-tetramethyl-2,2'-diphenyl-9H,9'H-9,9'-biimidazo[1,2-a]indole (dl-2e):

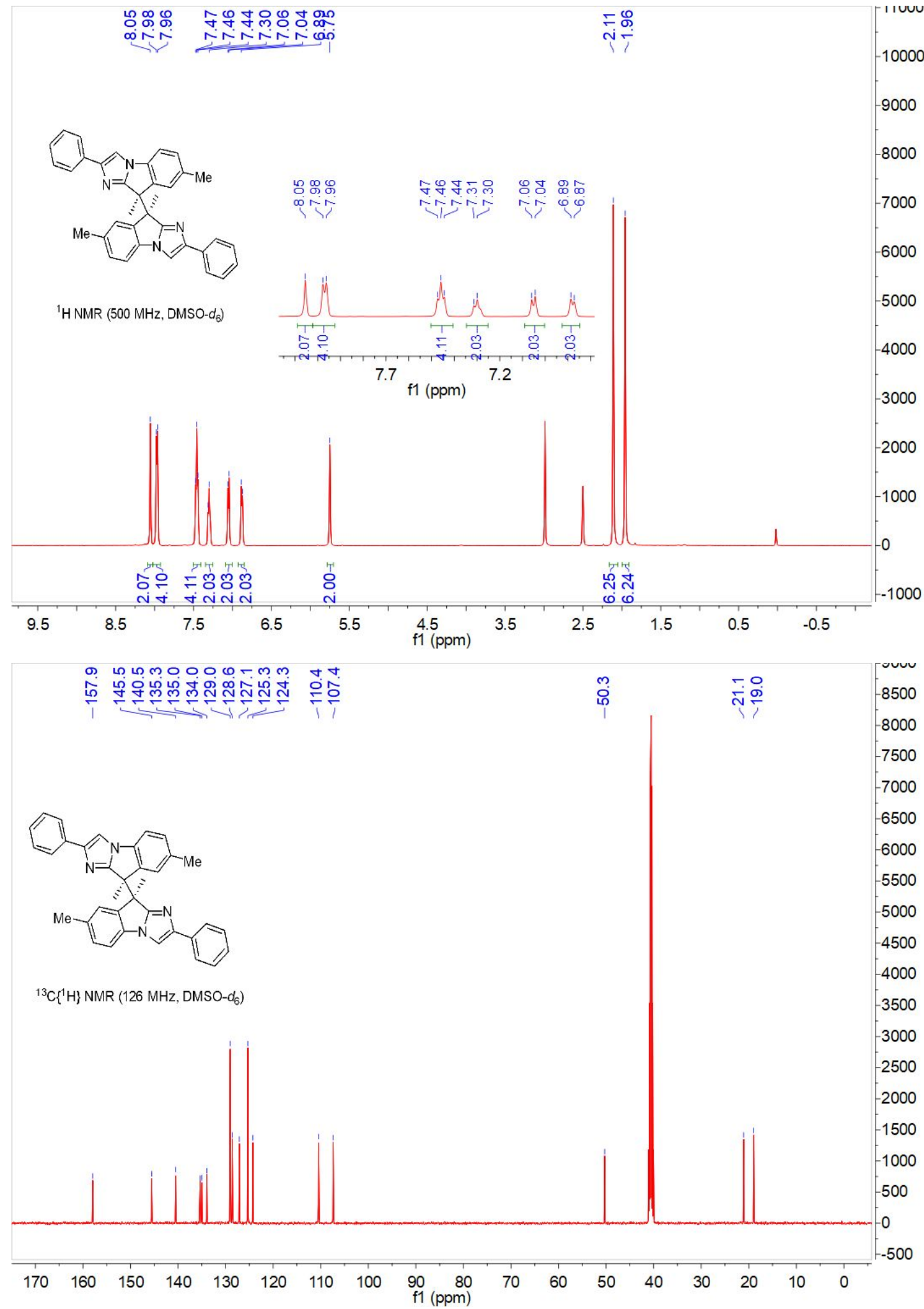


7,7',9,9'-tetramethyl-2,2'-diphenyl-9H,9'H-9,9'-biimidazo[1,2-a]indole (meso-2e):

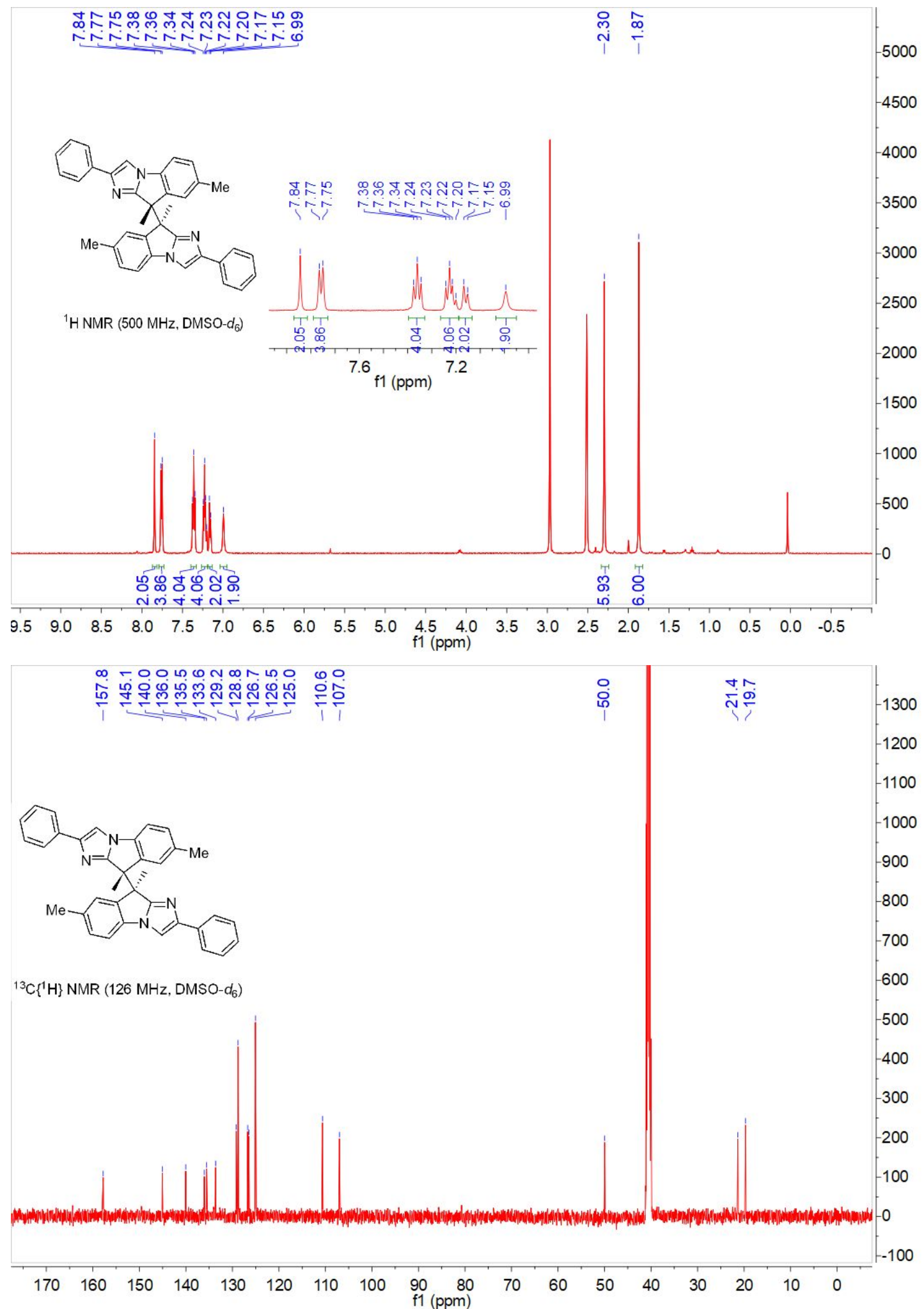


6,6',9,9'-tetramethyl-2,2'-diphenyl-9H,9'H-9,9'-biimidazo[1,2-a]indole (dl-2f):

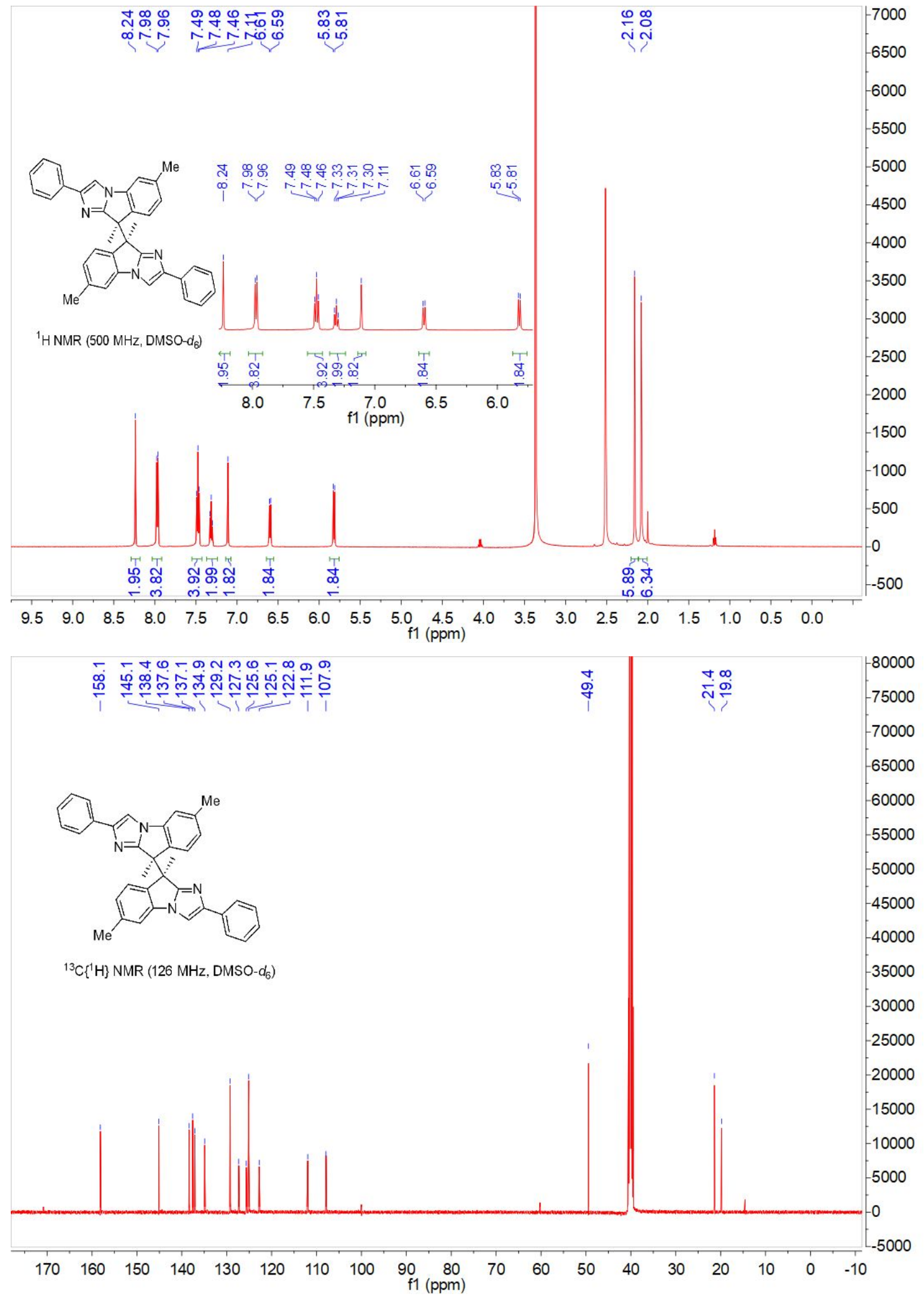


6,6',9,9'-tetramethyl-2,2'-diphenyl-9H,9'H-9,9'-biimidazo[1,2-a]indole (meso-2f):

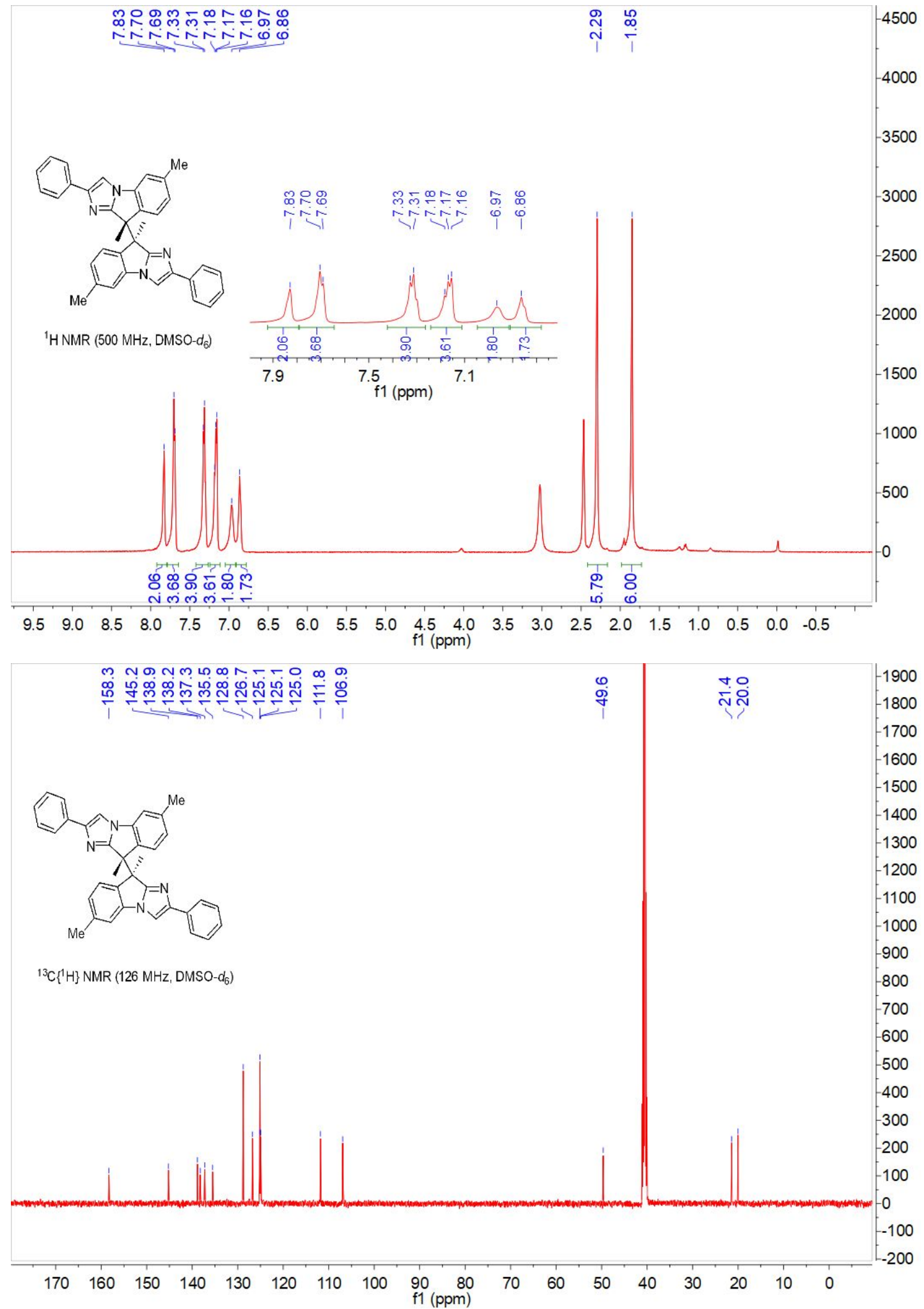


8,8'-dimethoxy-9,9'-dimethyl-2,2'-diphenyl-9H,9'H-9,9'-biimidazo[1,2-a]indole(meso-2g):

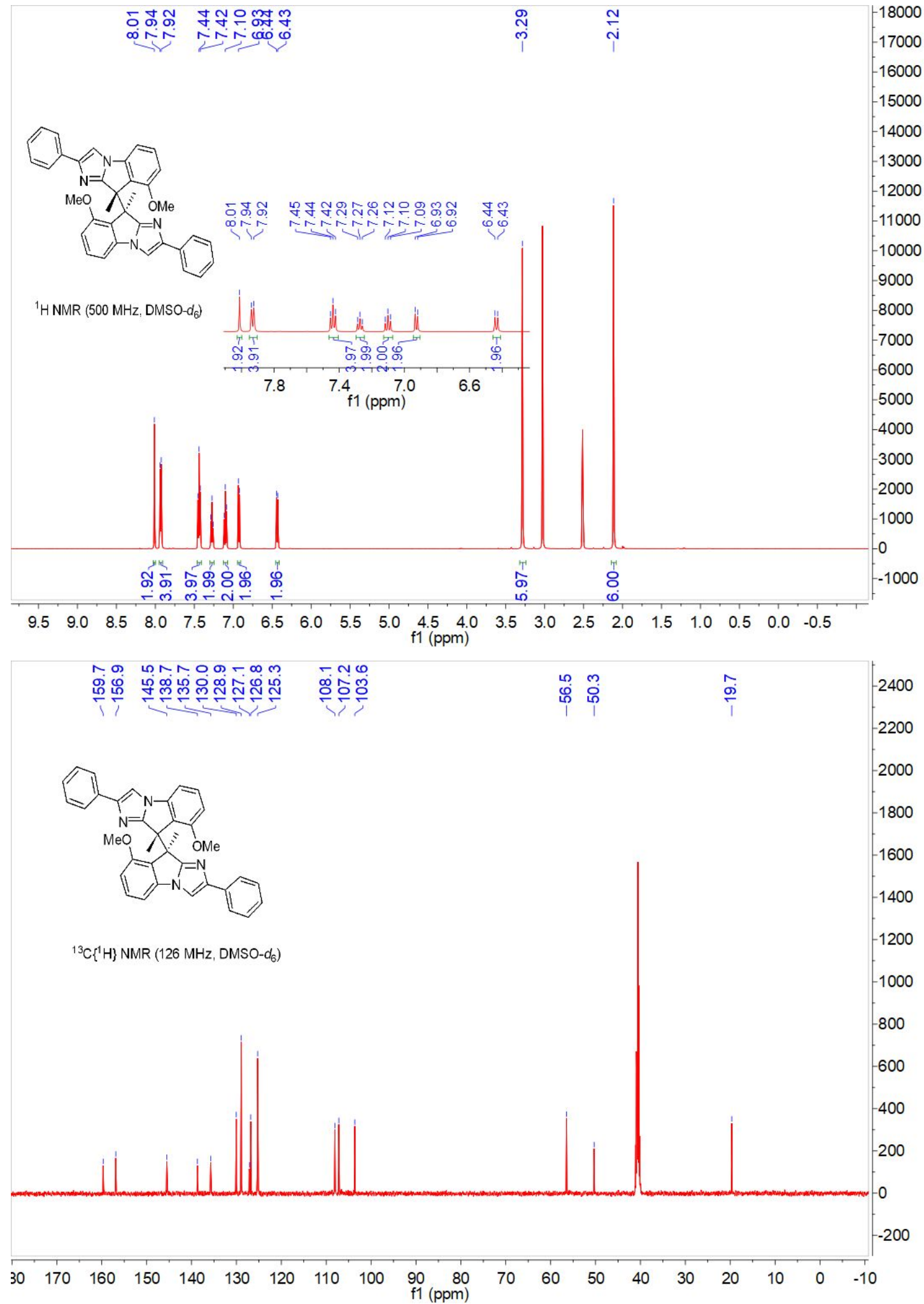


7,7'-dimethoxy-9,9'-dimethyl-2,2'-diphenyl-9H,9'H-9,9'-biimidazo[1,2-a]indole (dl-2h):




7,7'-dimethoxy-9,9'-dimethyl-2,2'-diphenyl-9H,9'H-9,9'-biimidazo[1,2-a]indole (meso-2h):

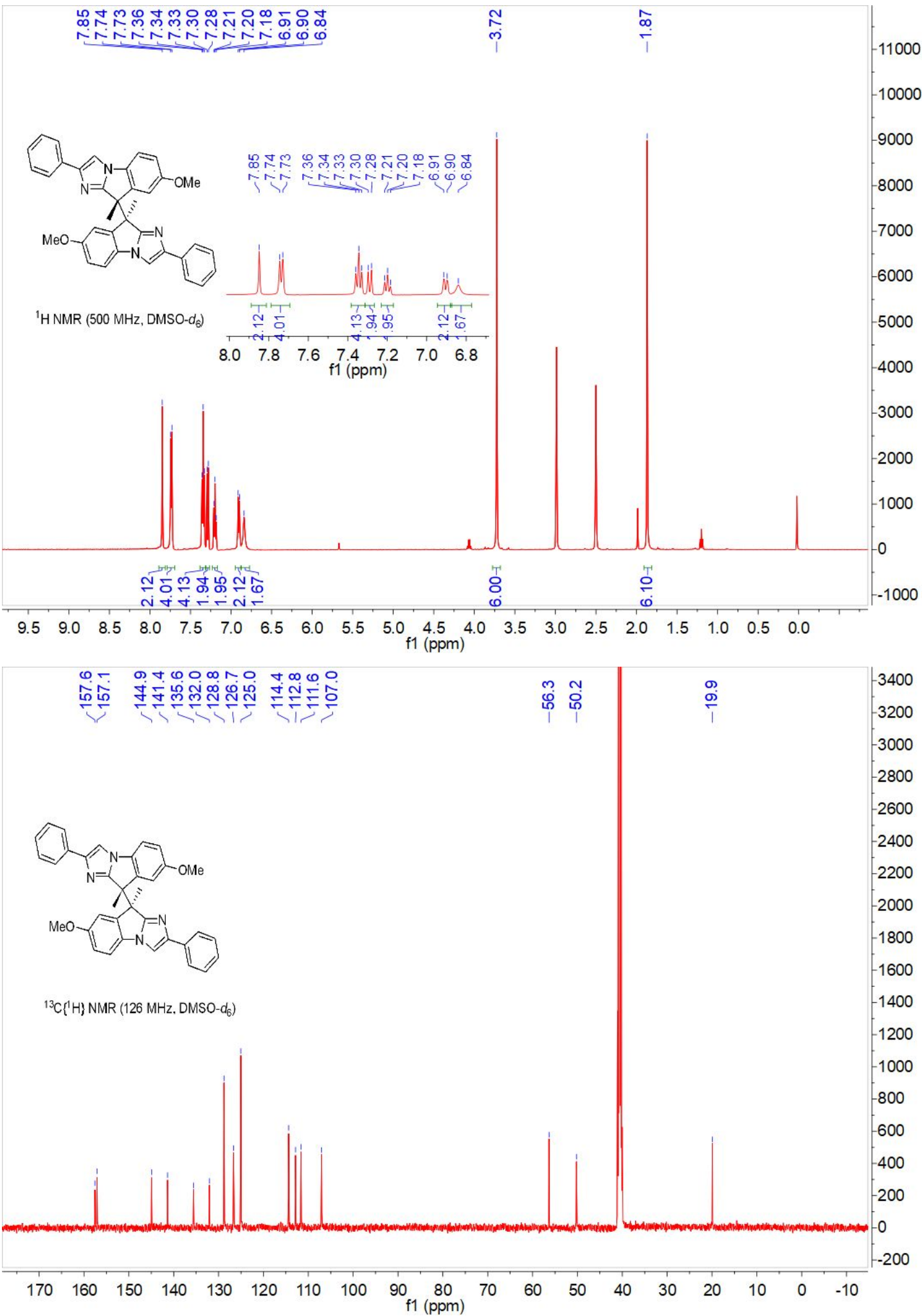


5,5'-dimethoxy-9,9'-dimethyl-2,2'-diphenyl-9H,9'H-9,9'-biimidazo[1,2-a]indole (dl-2i):

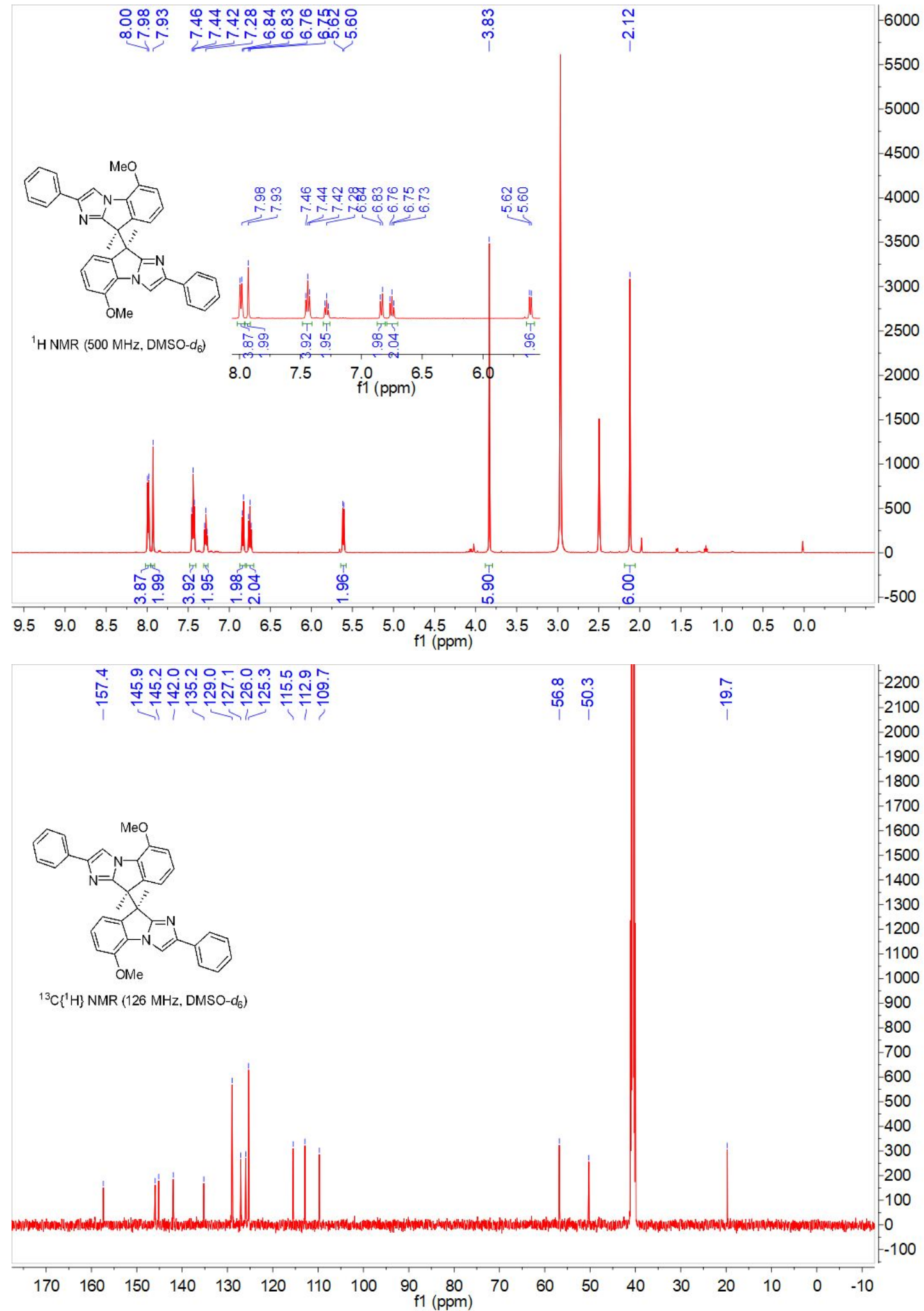


5,5'-dimethoxy-9,9'-dimethyl-2,2'-diphenyl-9H,9'H-9,9'-biimidazo[1,2-a]indole (meso-2i):

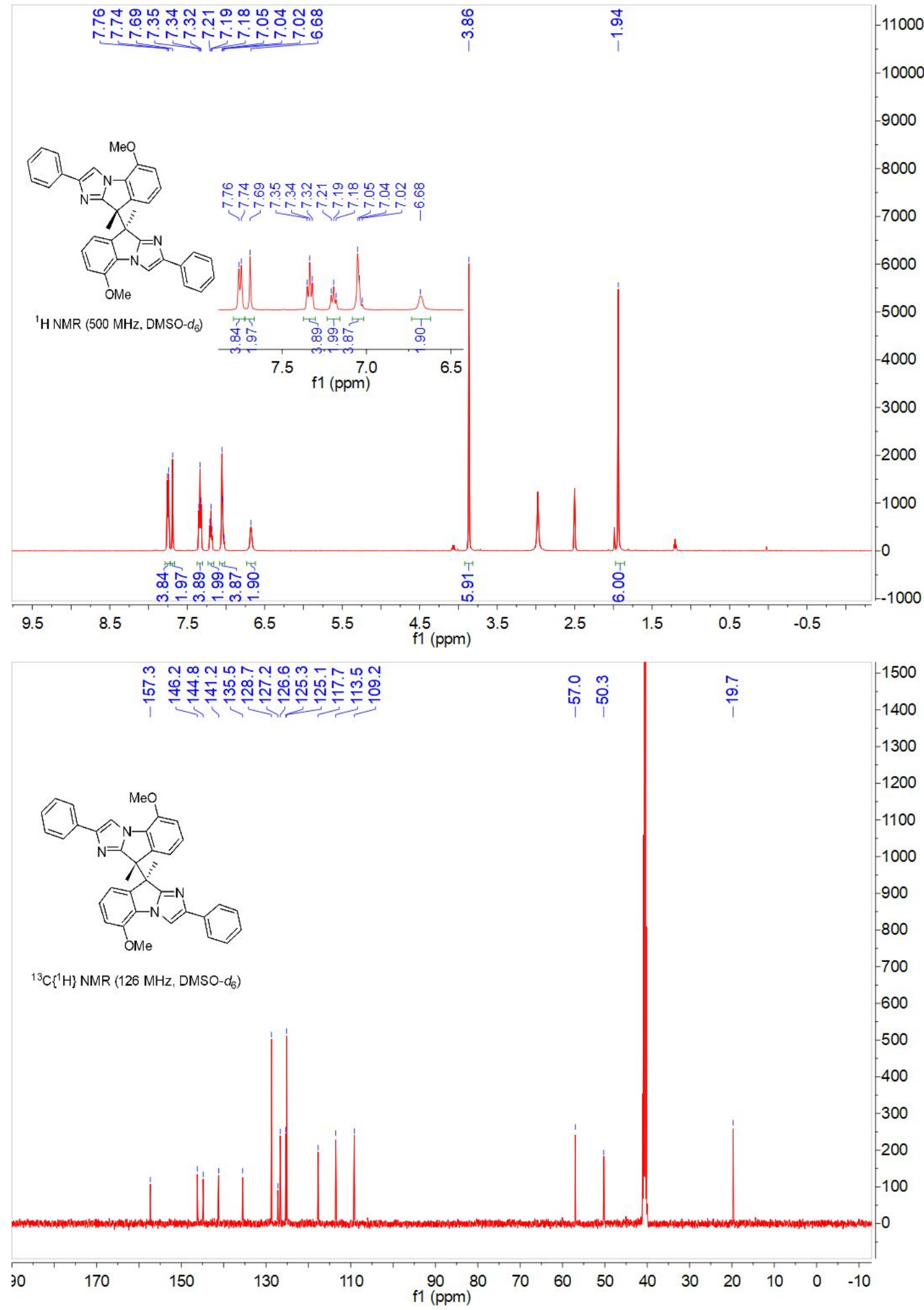


8,8'-difluoro-9,9'-dimethyl-2,2'-diphenyl-9H,9'H-9,9'-biimidazo[1,2-a]indole (dl-2j):

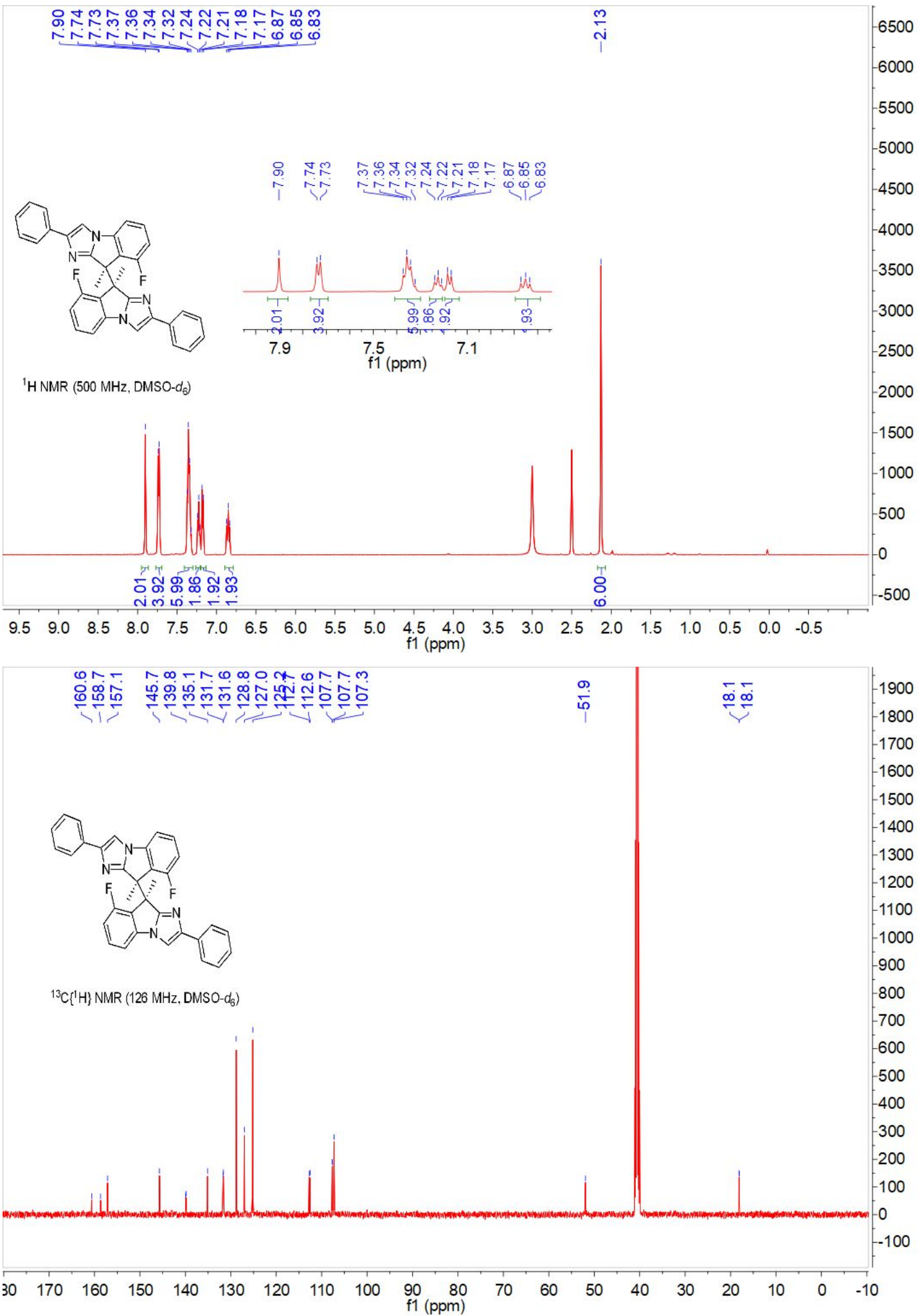


8,8'-difluoro-9,9'-dimethyl-2,2'-diphenyl-9H,9'H-9,9'-biimidazo[1,2-a]indole (meso-2j):

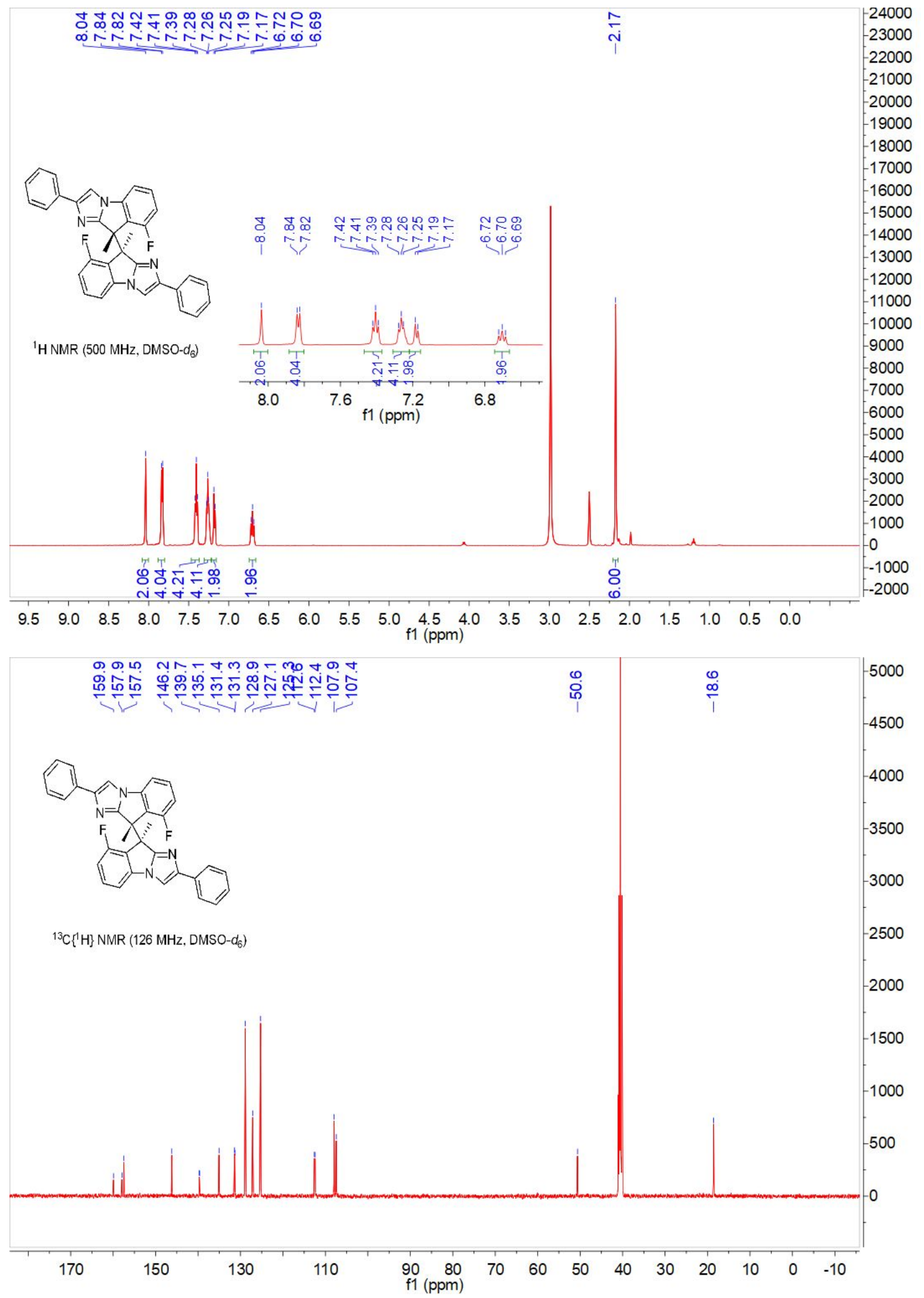


6,6'-dichloro-9,9'-dimethyl-2,2'-diphenyl-9H,9'H-9,9'-biimidazo[1,2-a]indole ( $d l-2 \mathrm{k})$ :

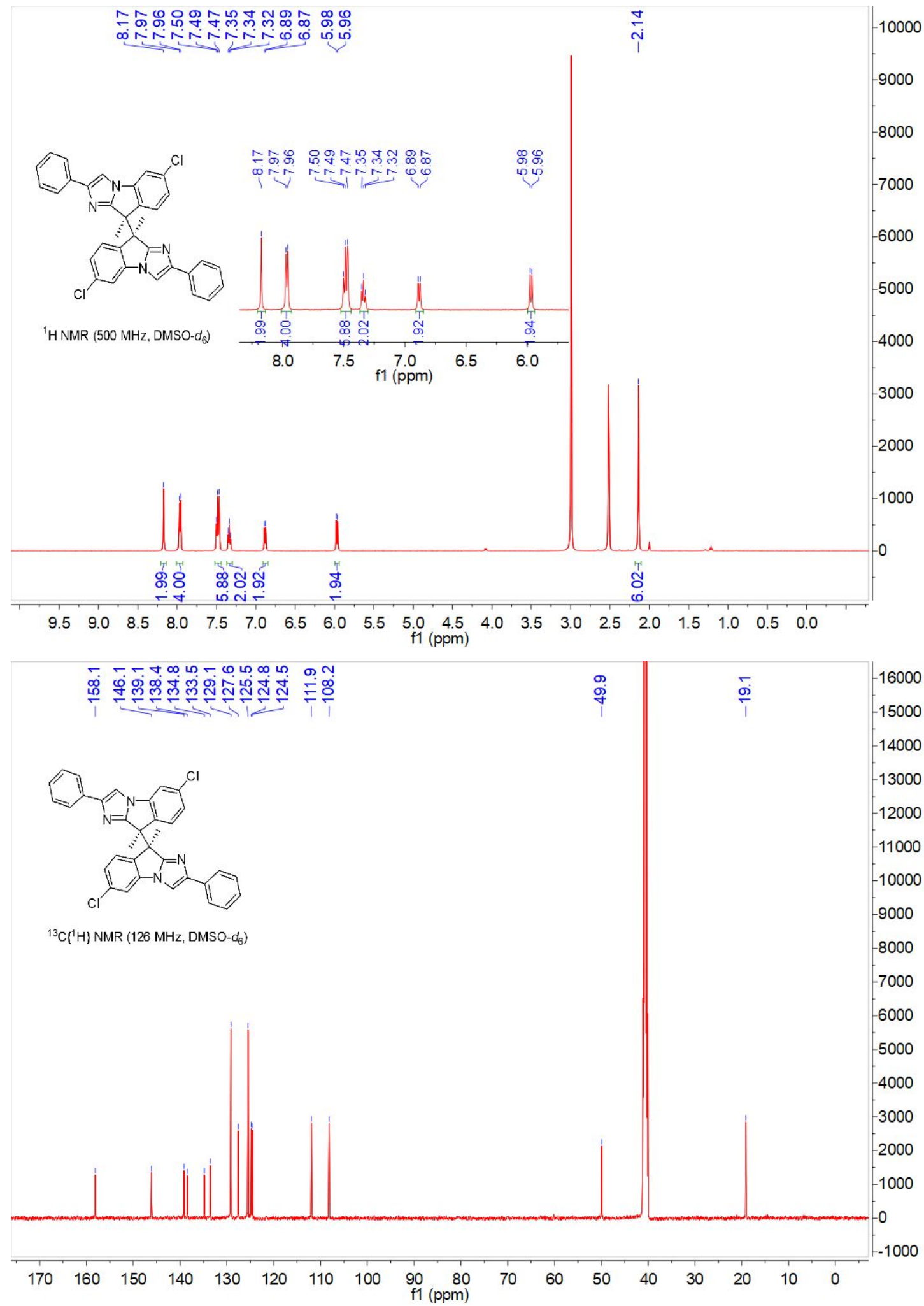


6,6'-dichloro-9,9'-dimethyl-2,2'-diphenyl-9H,9'H-9,9'-biimidazo[1,2-a]indole (meso-2k):

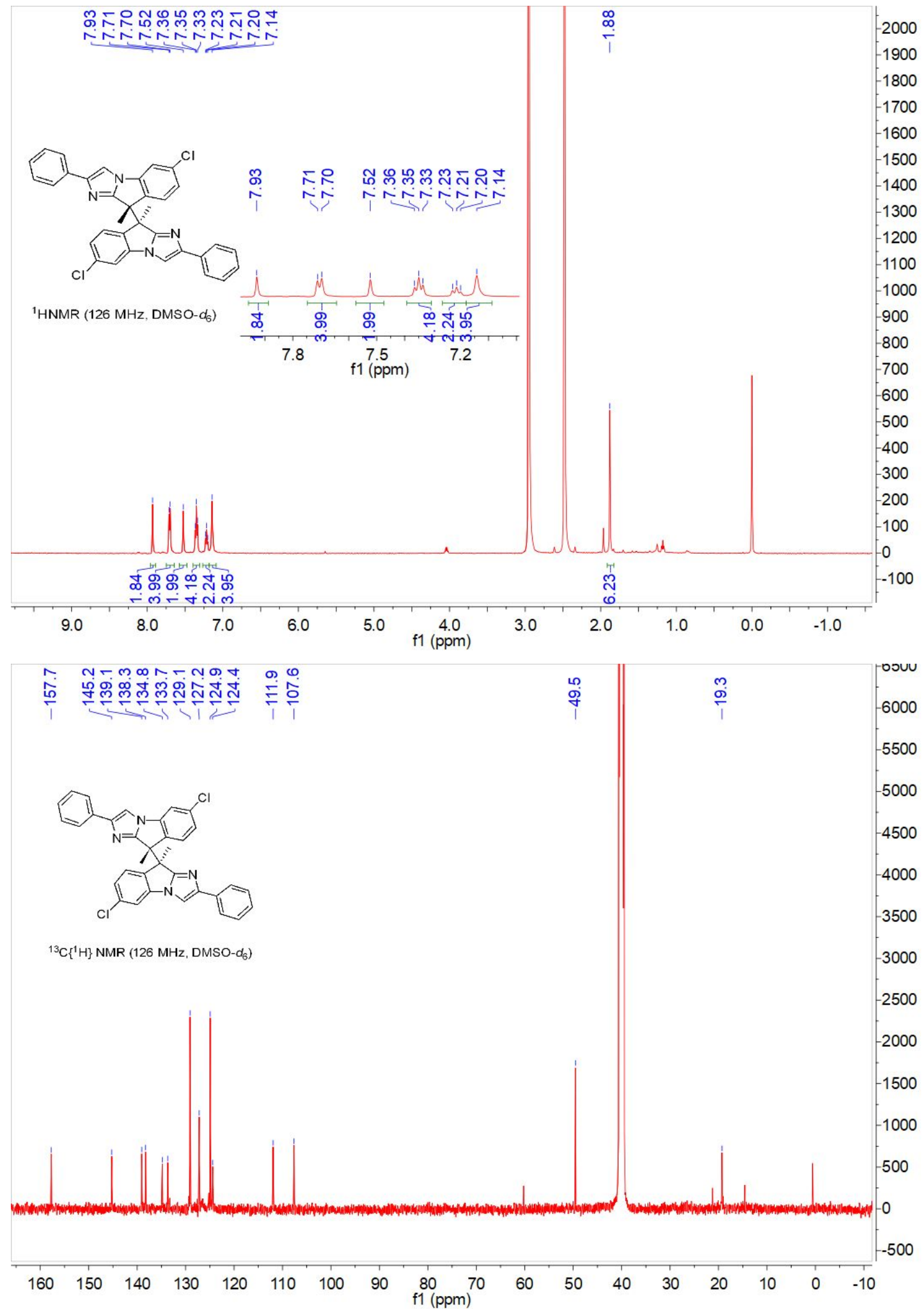


6,6'-dibromo-9,9'-dimethyl-2,2'-diphenyl-9H,9'H-9,9'-biimidazo[1,2-a]indole (dl-2l):

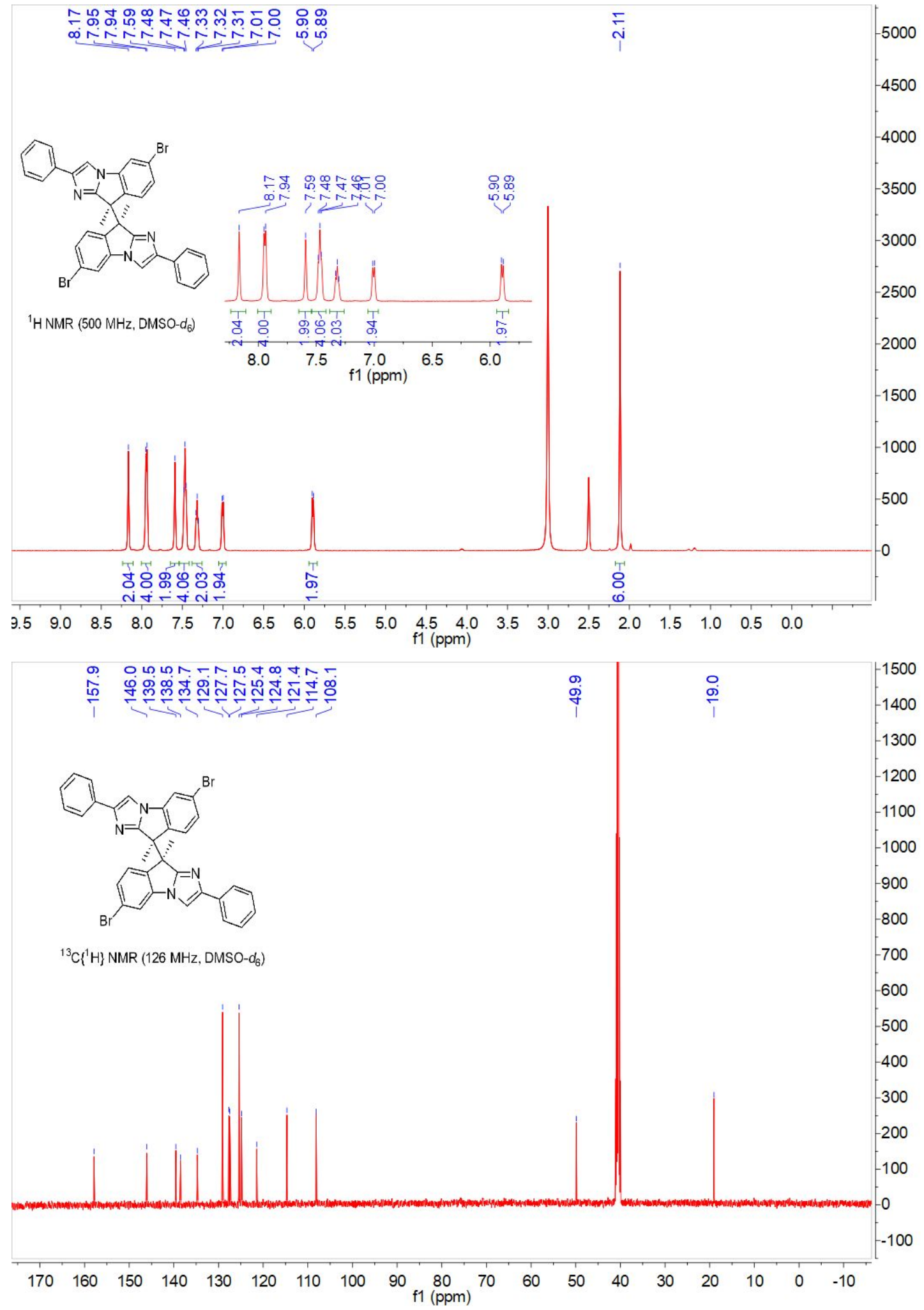


6,6'-dibromo-9,9'-dimethyl-2,2'-diphenyl-9H,9'H-9,9'-biimidazo[1,2-a]indole (meso-2l):

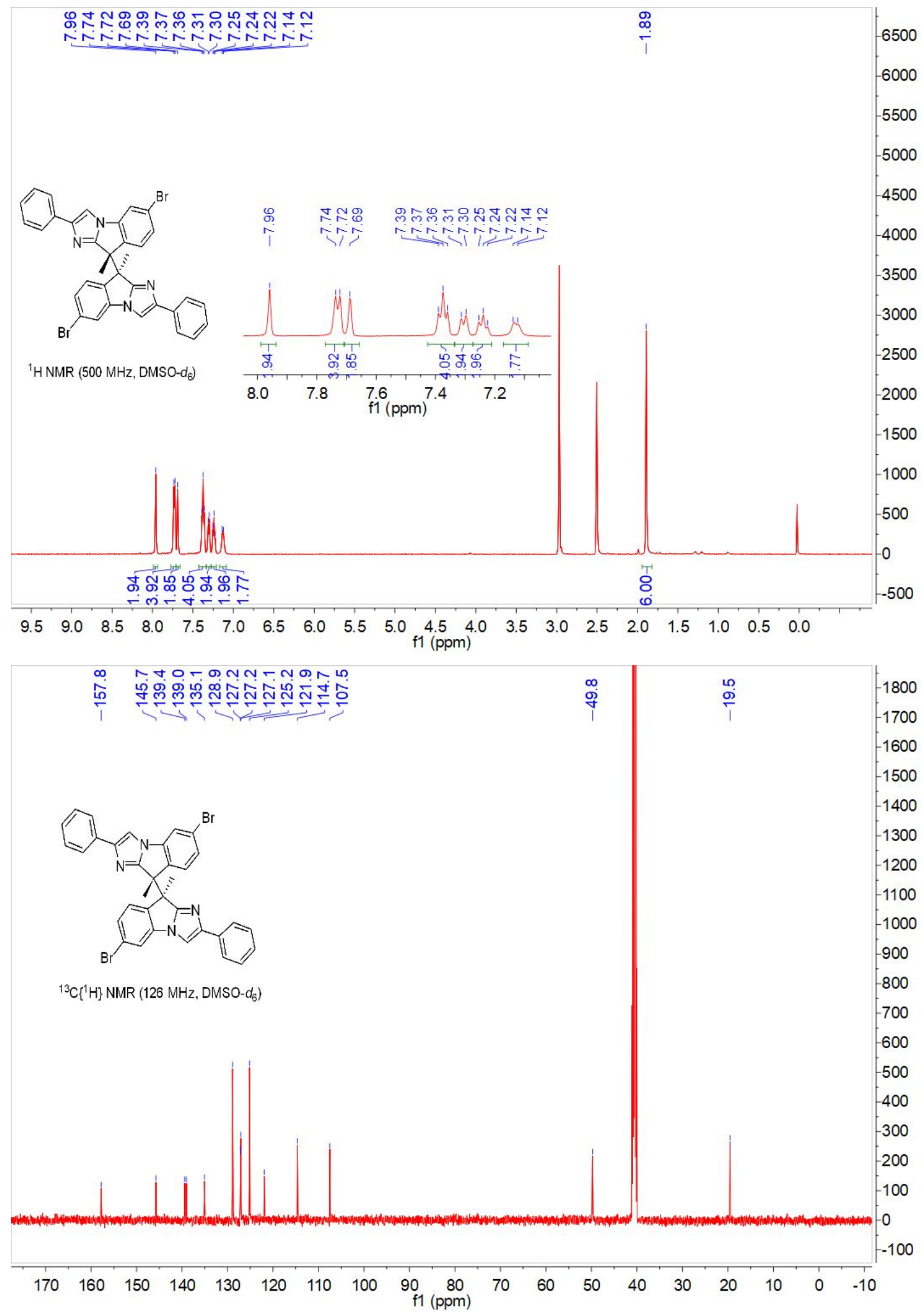


5,5'-dibromo-9,9'-dimethyl-2,2'-diphenyl-9H,9'H-9,9'-biimidazo[1,2-a]indole (dl-2m):

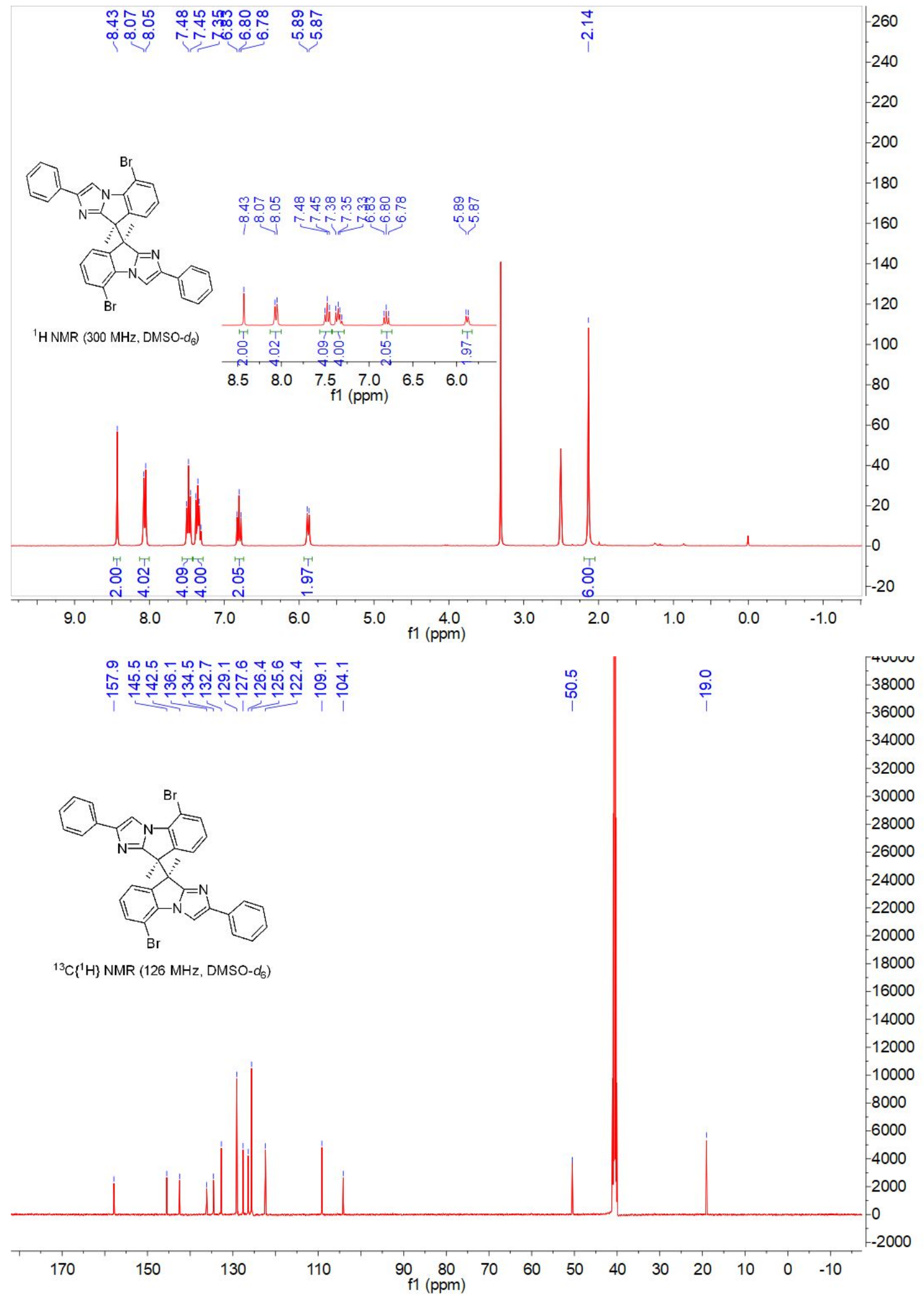


5,5'-dibromo-9,9'-dimethyl-2,2'-diphenyl-9H,9'H-9,9'-biimidazo[1,2-a]indole(meso-2m):

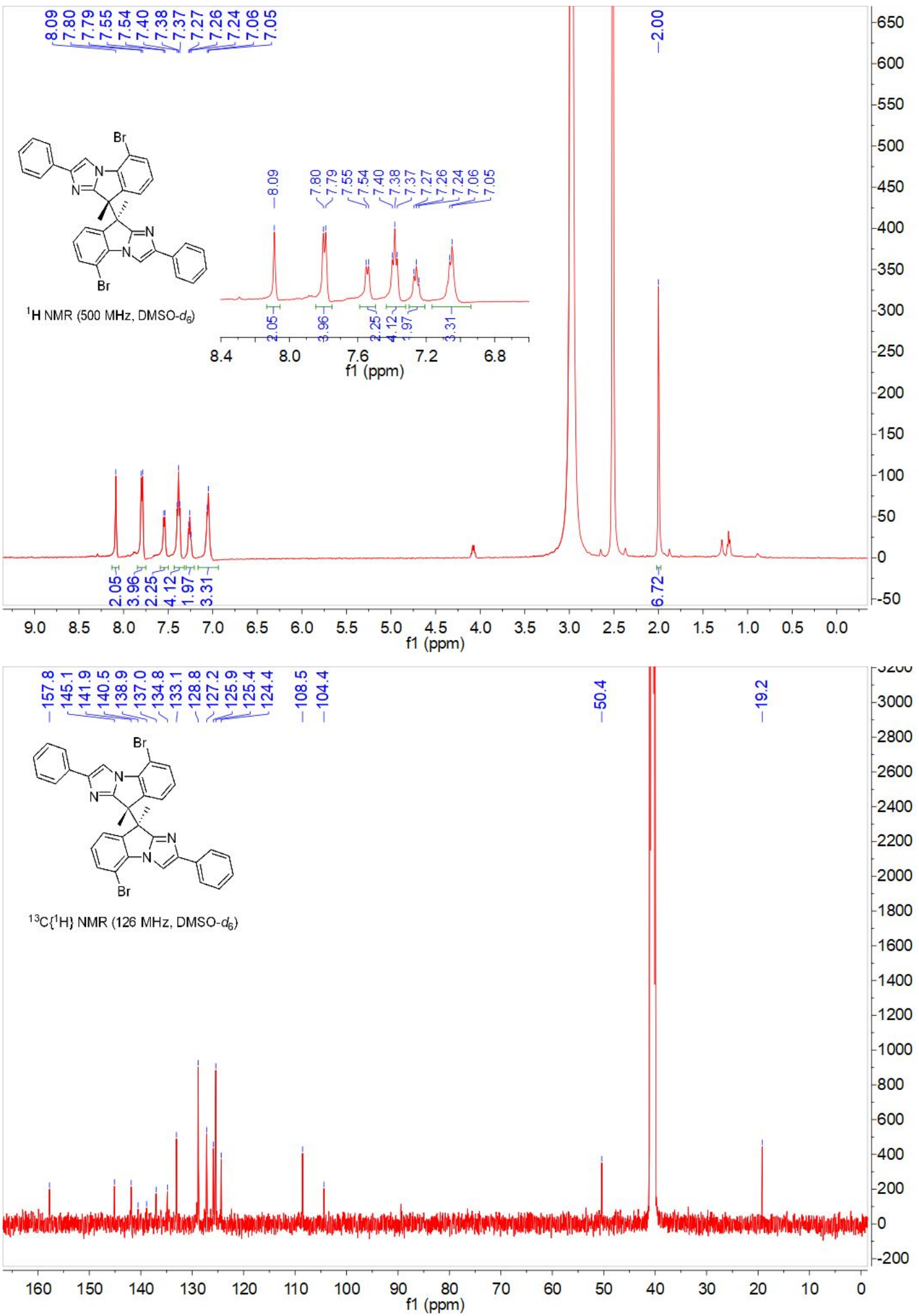


9,9'-dimethyl-2,2'-di-p-tolyl-9H,9'H-9,9'-biimidazo[1,2-a]indole (dl-2n):

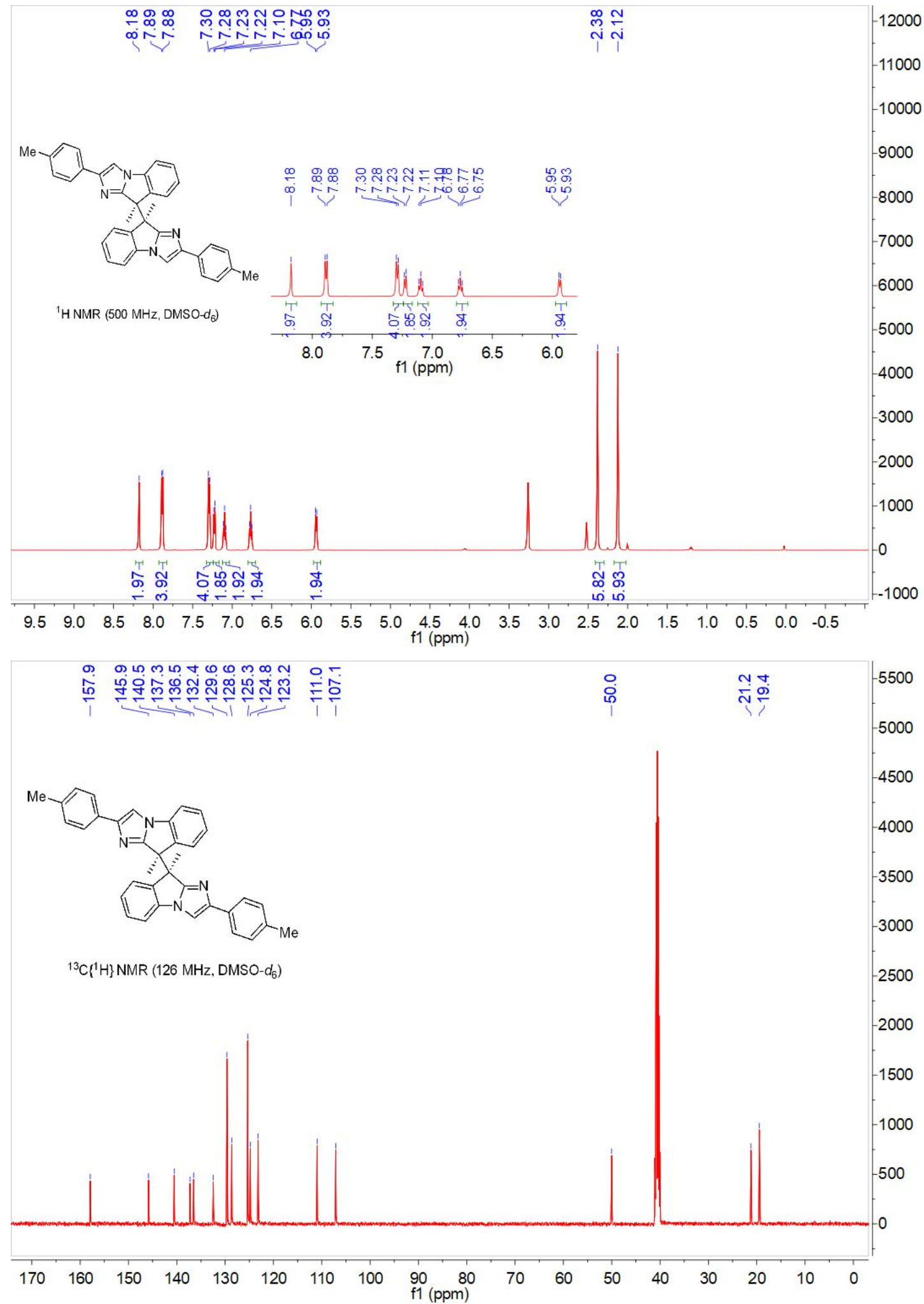


9,9'-dimethyl-2,2'-di-p-tolyl-9H,9'H-9,9'-biimidazo[1,2-a]indole (meso-2n):

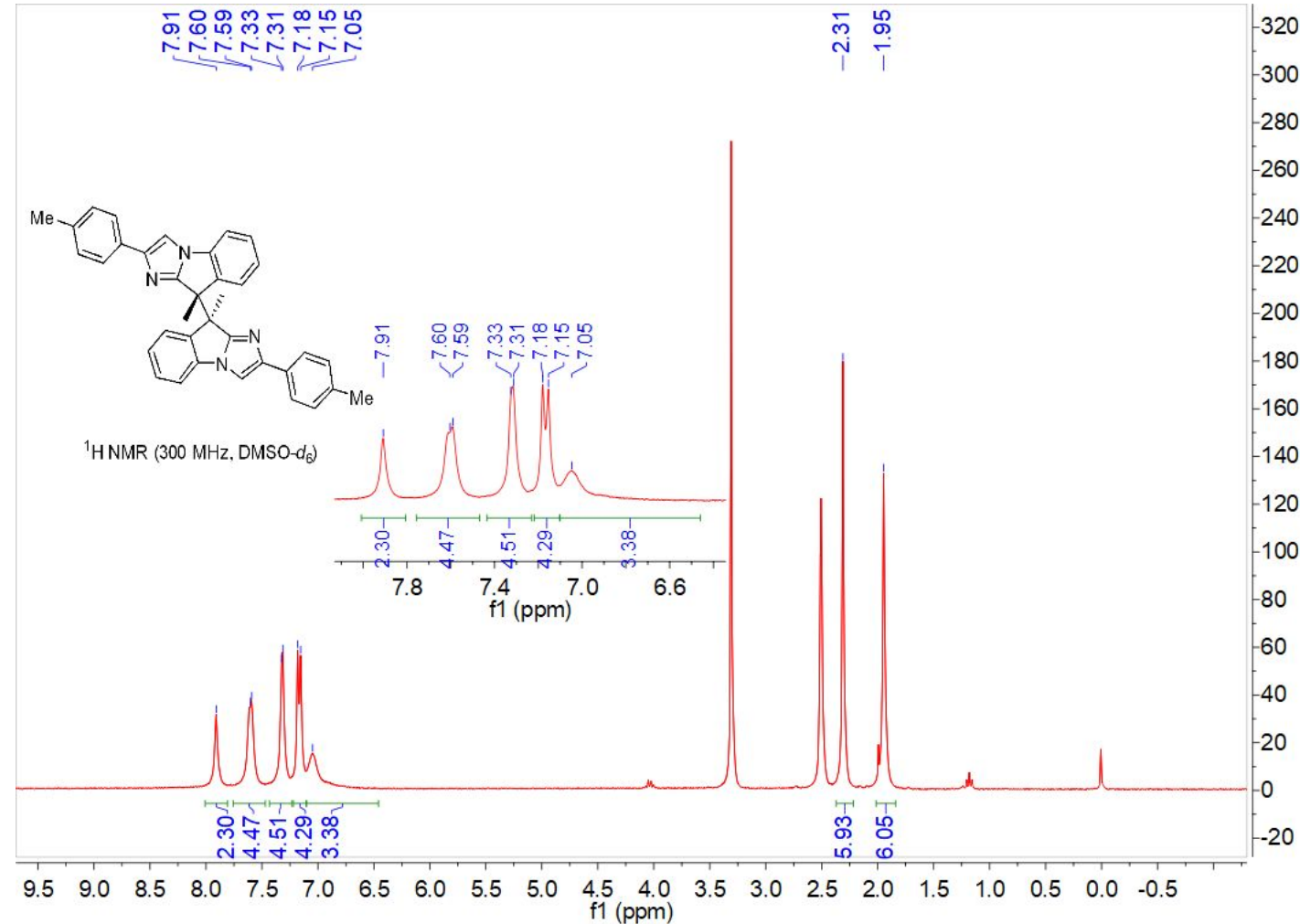

둔
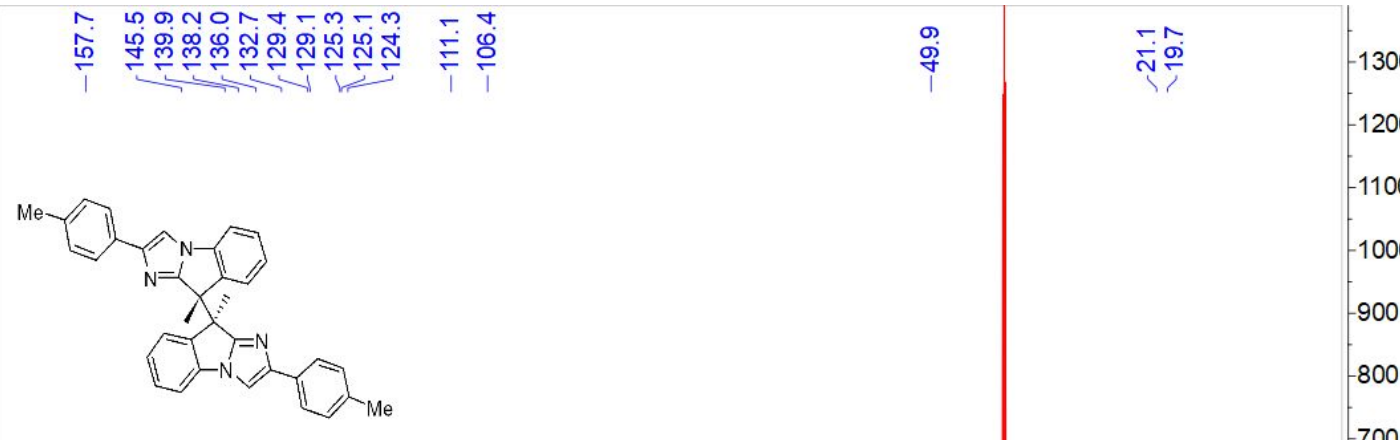

${ }^{13} \mathrm{C}\{\mathrm{H} H\}$ NMR (126 MHz, DMSO- $\left.d_{6}\right)$

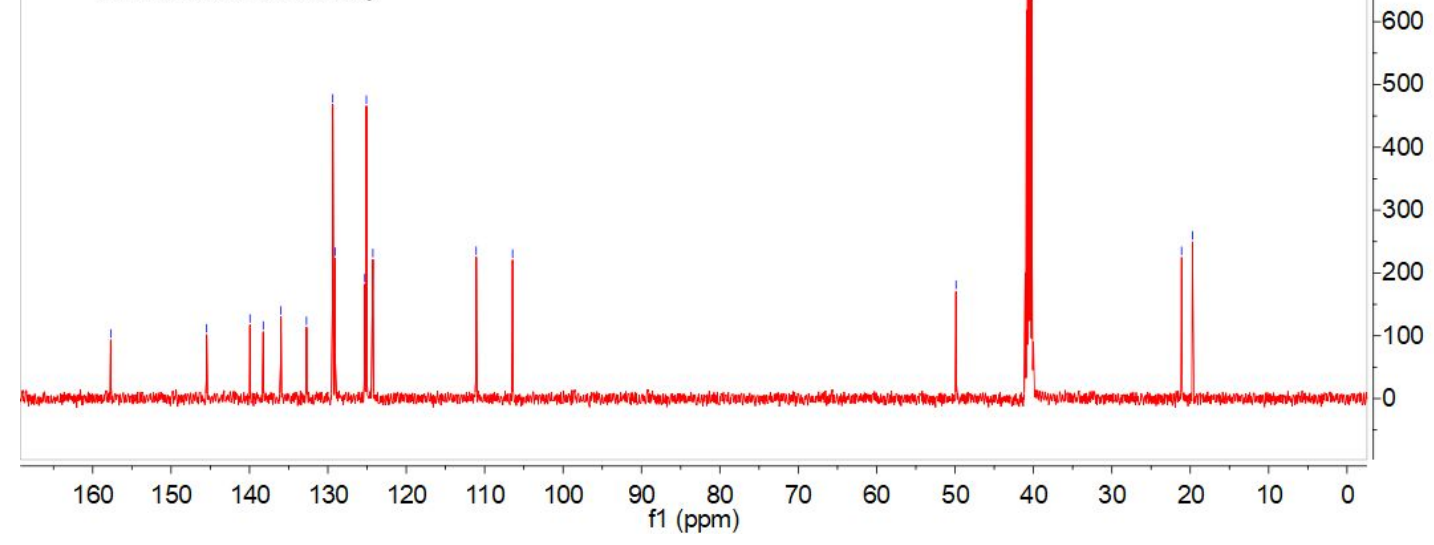


2,2'-bis(3-methoxyphenyl)-9,9'-dimethyl-9H,9'H-9,9'-biimidazo[1,2-a]indole ( $d l-20)$ :

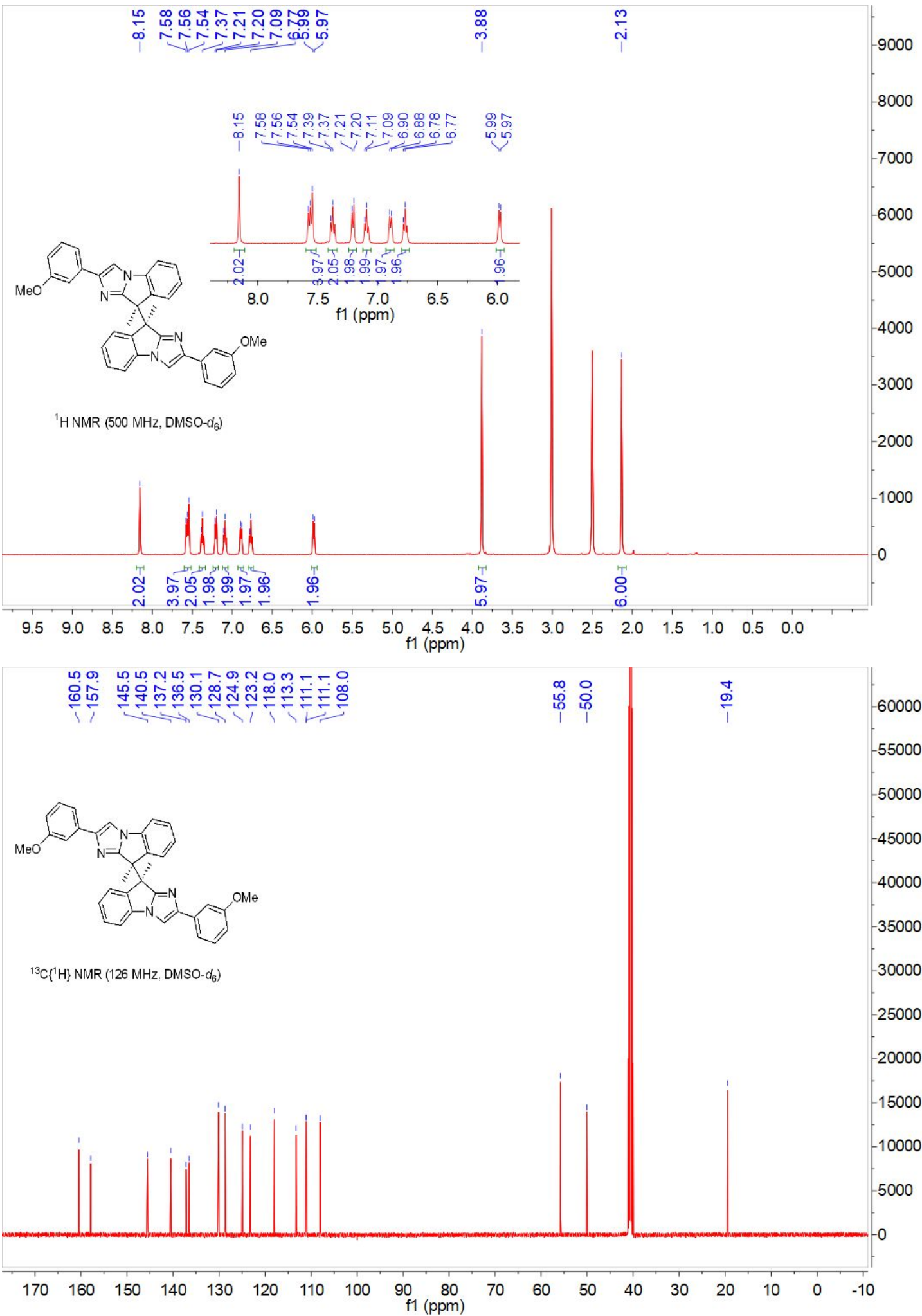


2,2'-bis(3-methoxyphenyl)-9,9'-dimethyl-9H,9'H-9,9'-biimidazo[1,2-a]indole (meso-2o):

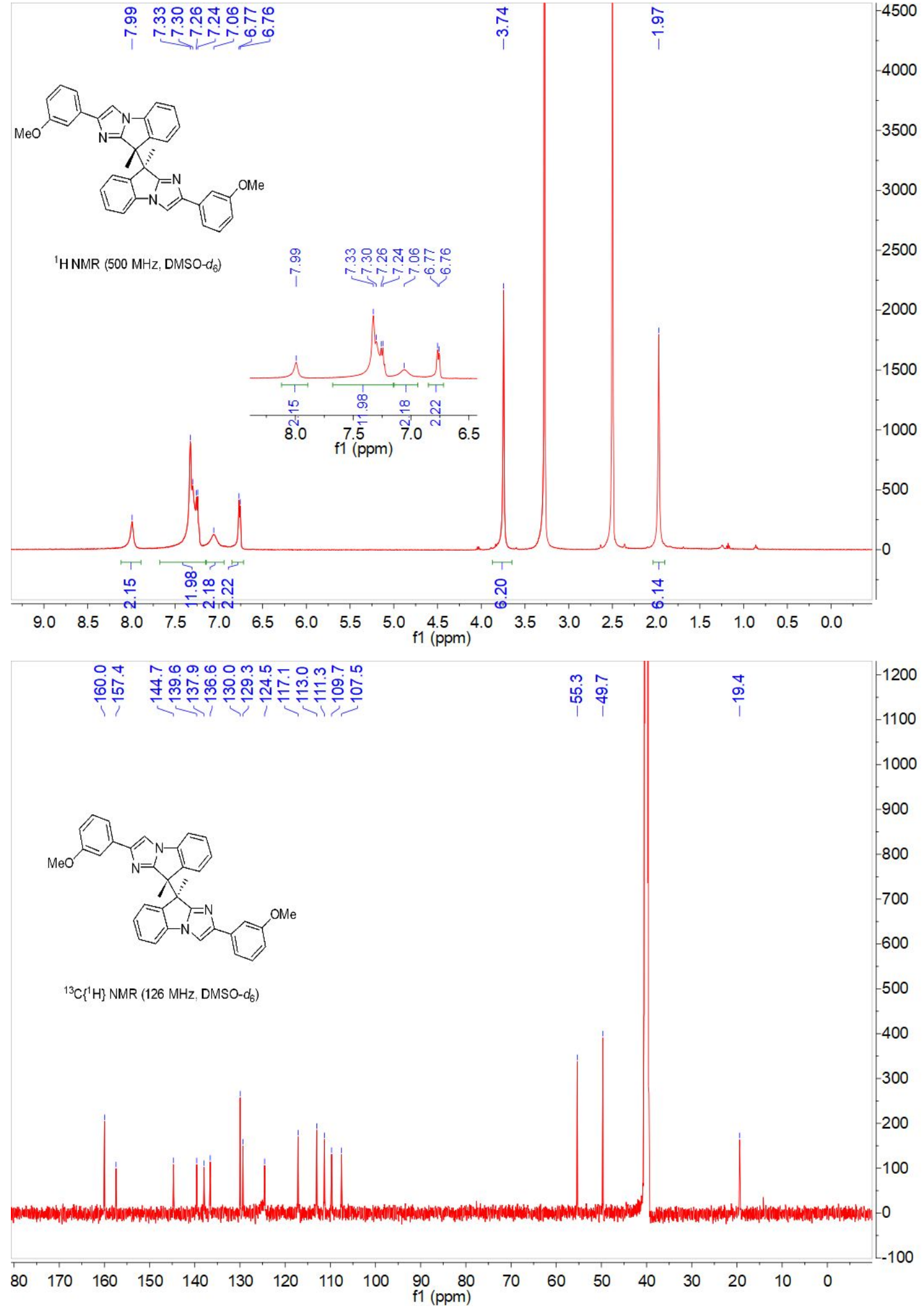


2,2'-bis(4-methoxyphenyl)-9,9'-dimethyl-9H,9'H-9,9'-biimidazo[1,2-a]indole (dl-2p):

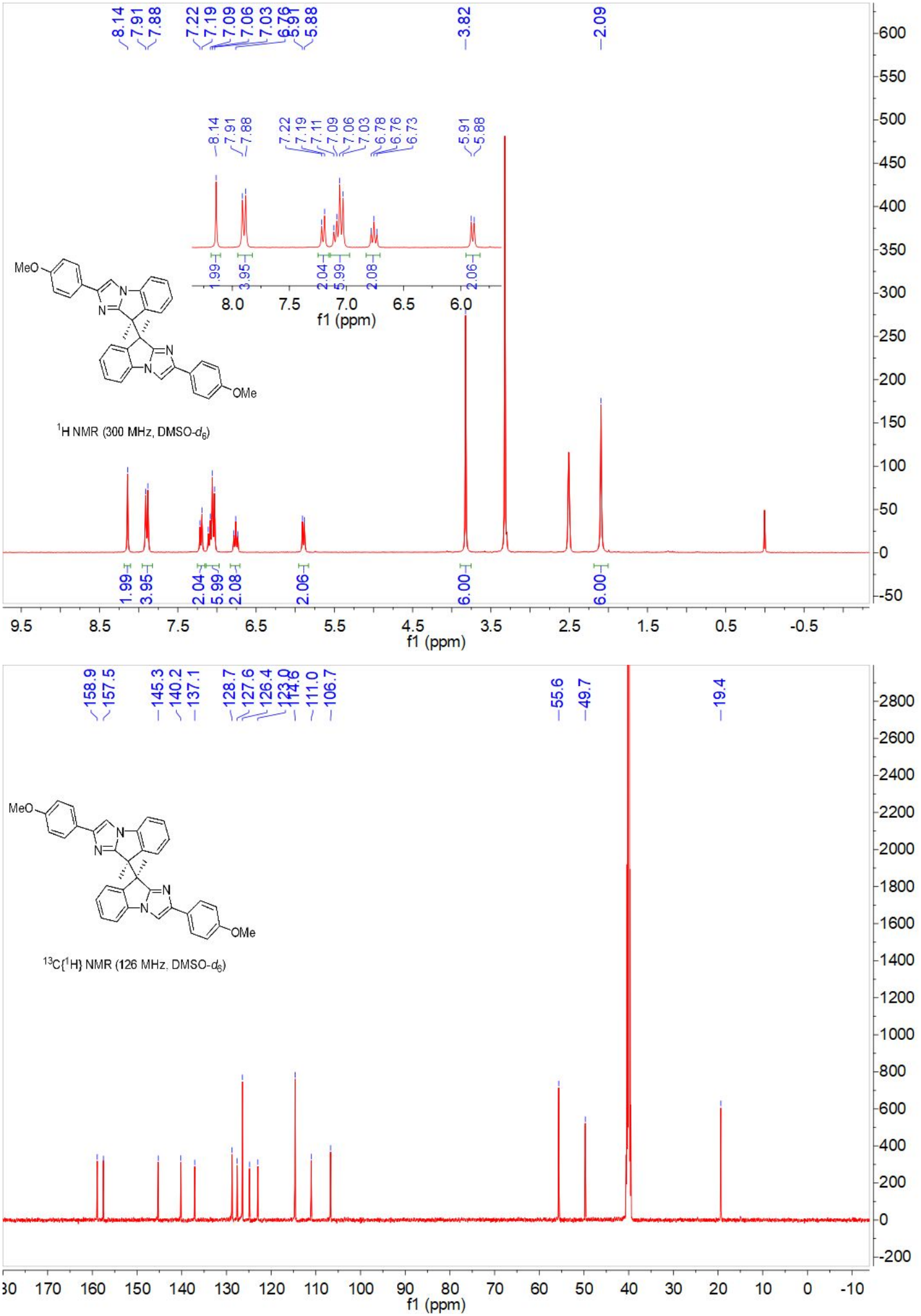


2,2'-bis(4-methoxyphenyl)-9,9'-dimethyl-9H,9'H-9,9'-biimidazo[1,2-a]indole (meso-2p):

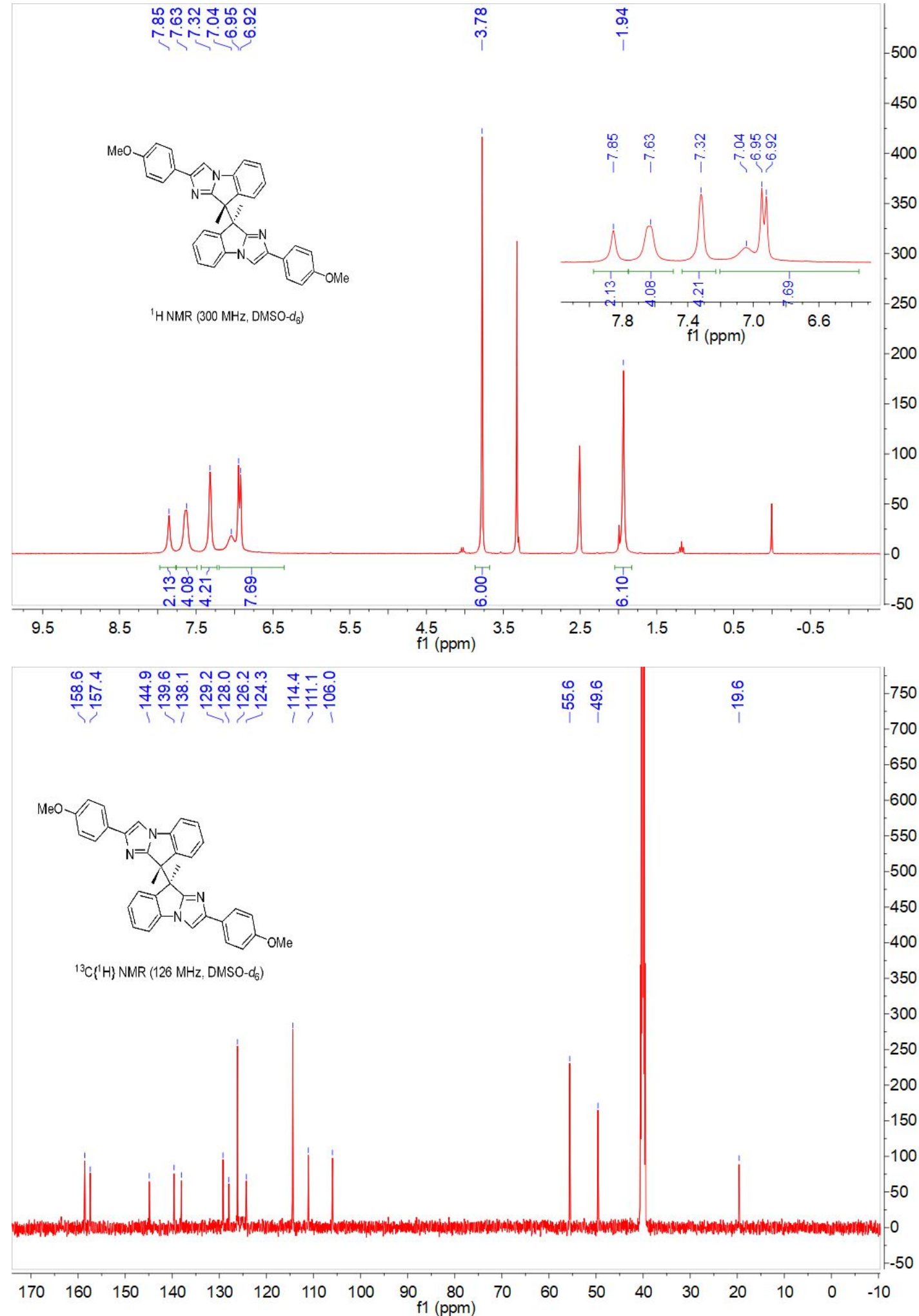


2,2'-bis(2-fluorophenyl)-9,9'-dimethyl-9H,9'H-9,9'-biimidazo[1,2-a]indole (dl-2q):

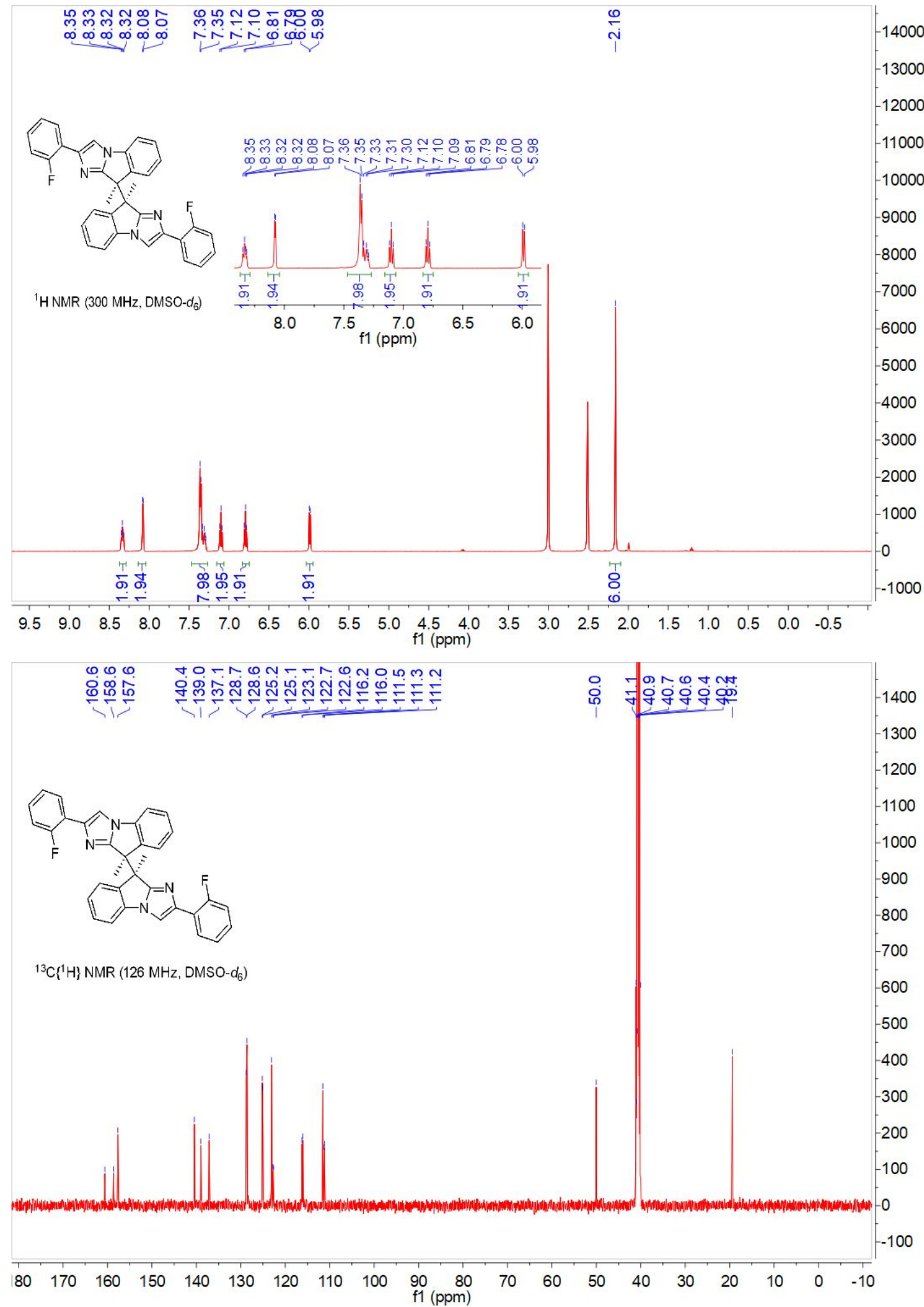


2,2'-bis(2-fluorophenyl)-9,9'-dimethyl-9H,9'H-9,9'-biimidazo[1,2-a]indole (meso-2q):
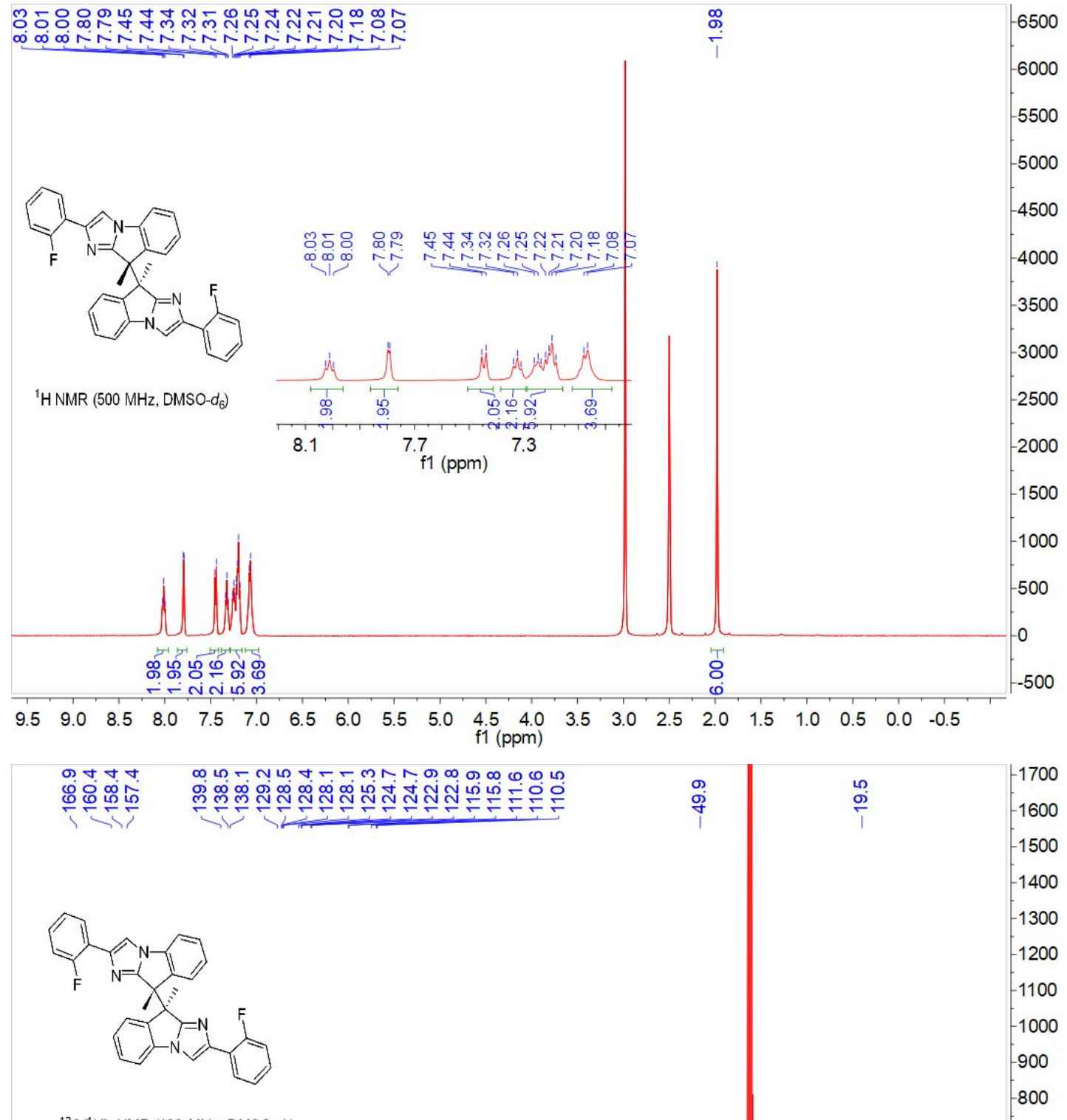

${ }^{13} \mathrm{C}\left\{{ }^{1} \mathrm{H}\right\}$ NMR (126 MHz, DMSO-d $)$

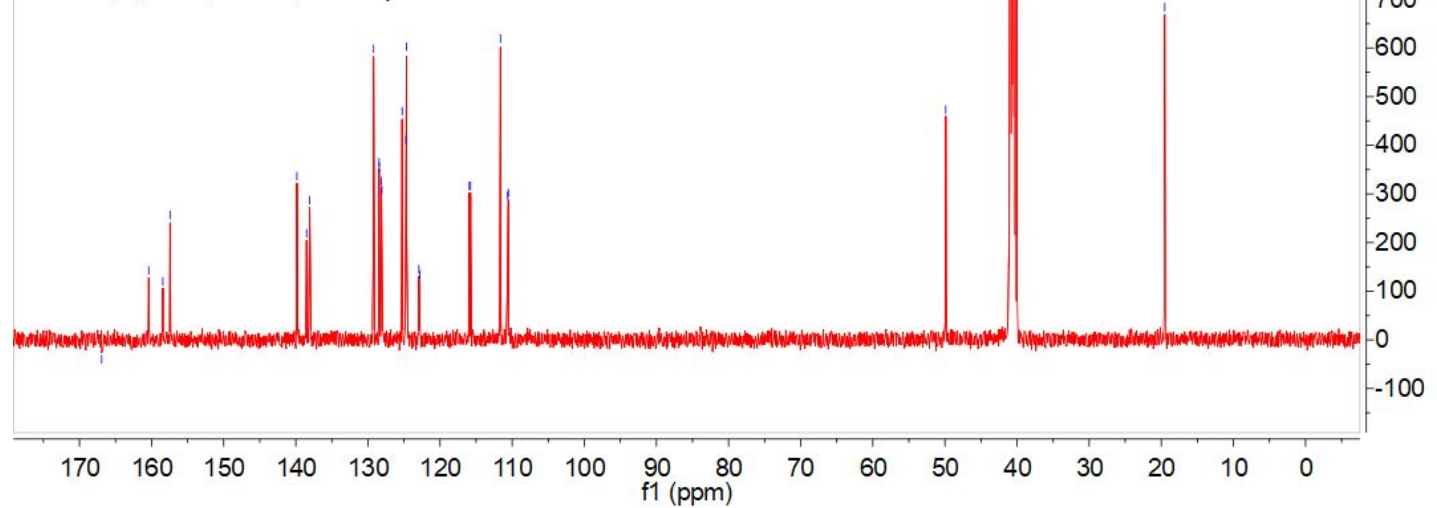


2,2'-bis(3-fluorophenyl)-9,9'-dimethyl-9H,9'H-9,9'-biimidazo[1,2-a]indole (dl-2r):

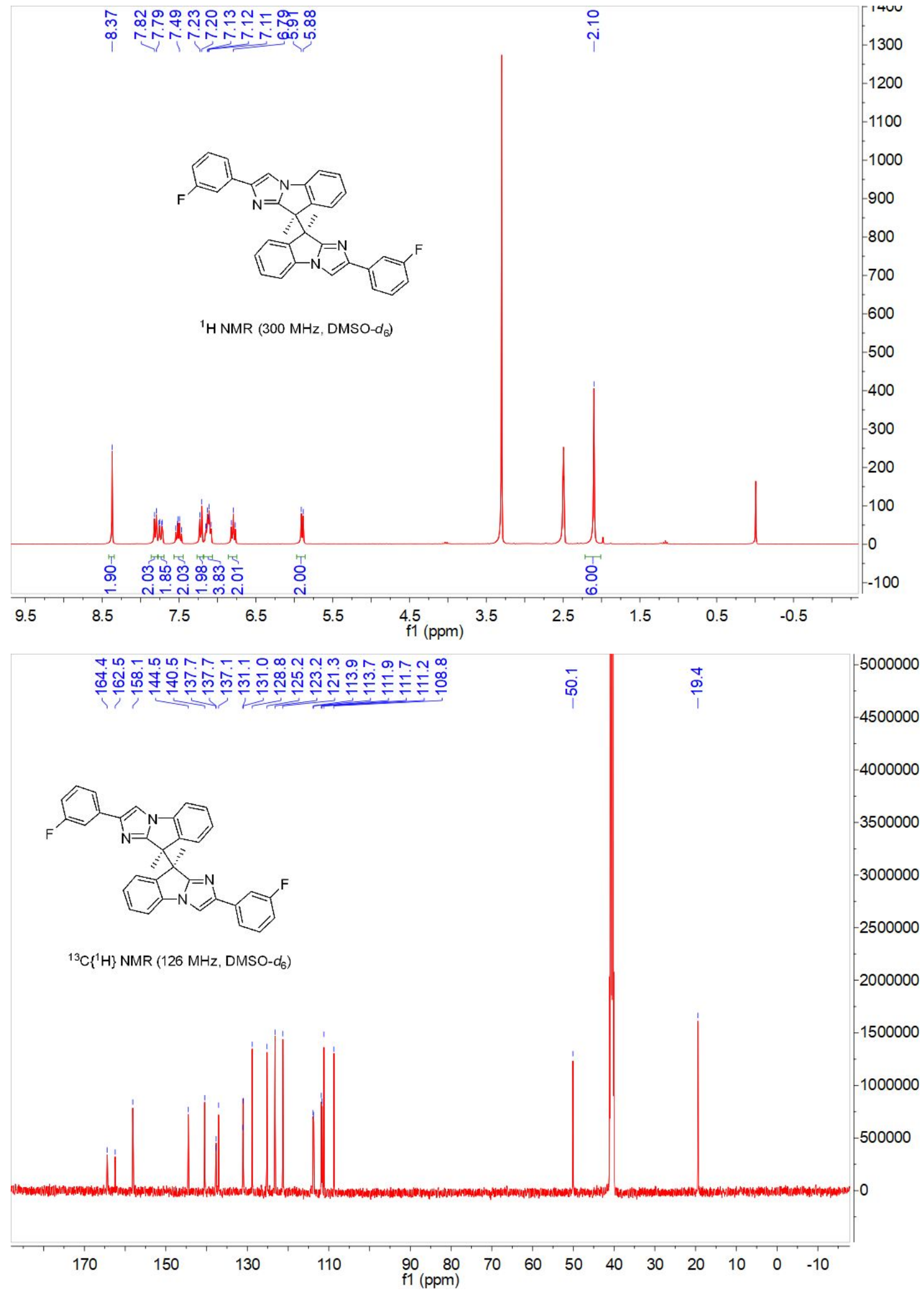


2,2'-bis(3-fluorophenyl)-9,9'-dimethyl-9H,9'H-9,9'-biimidazo[1,2-a]indole (meso-2r):

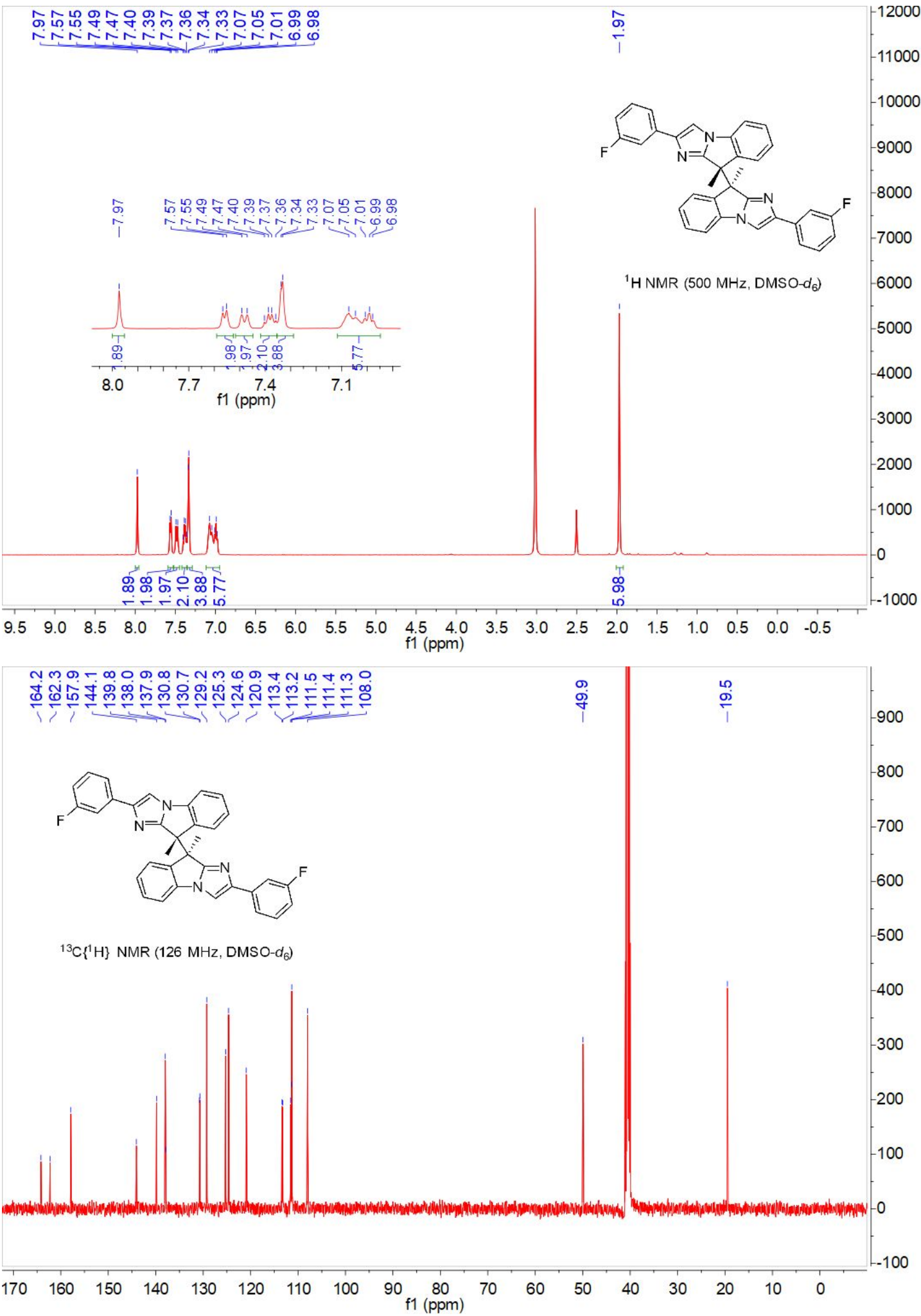


2,2'-bis(4-fluorophenyl)-9,9'-dimethyl-9H,9'H-9,9'-biimidazo[1,2-a]indole ( $d l-2 s)$ :

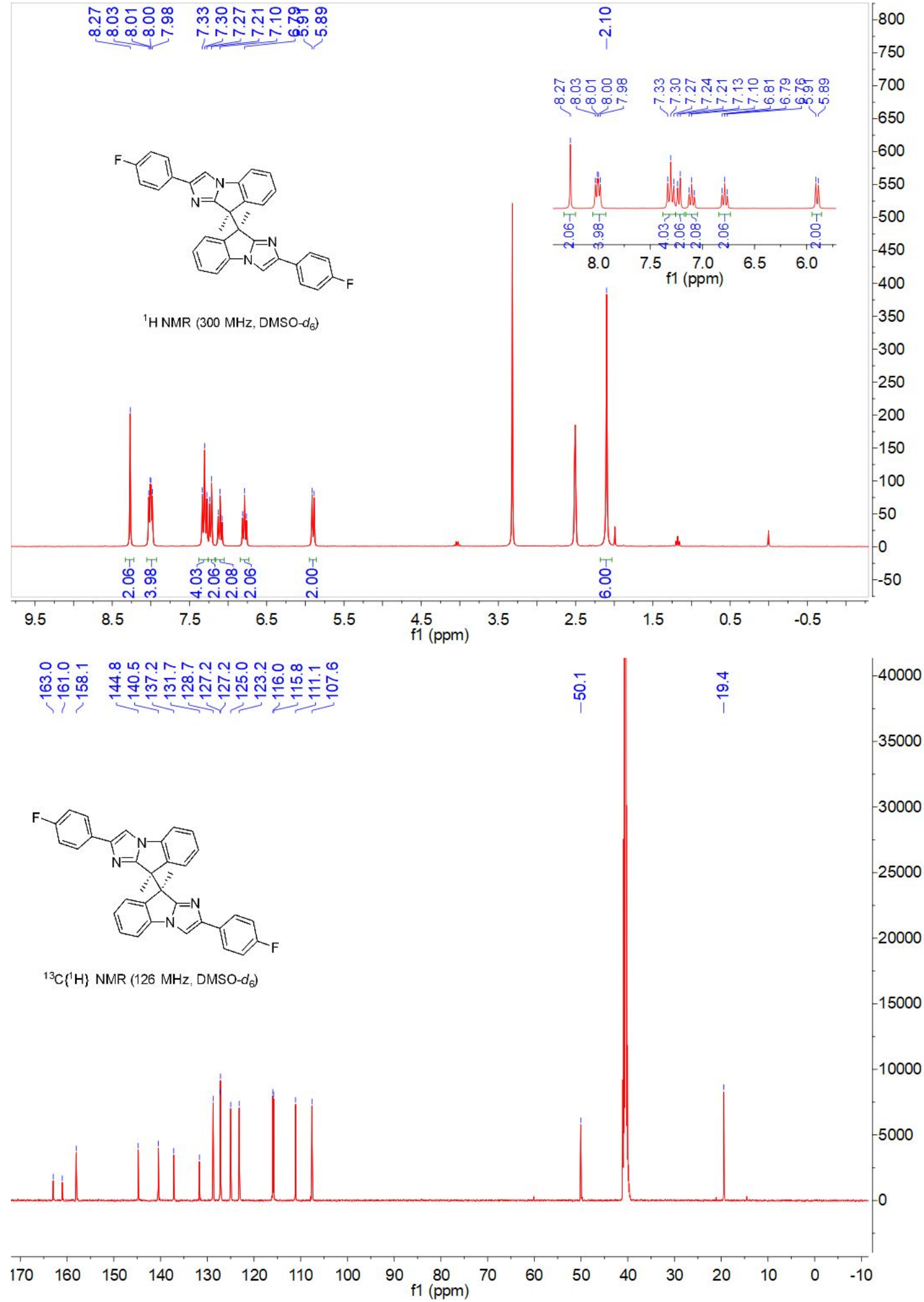


2,2'-bis(4-fluorophenyl)-9,9'-dimethyl-9H,9'H-9,9'-biimidazo[1,2-a]indole (meso-2s):

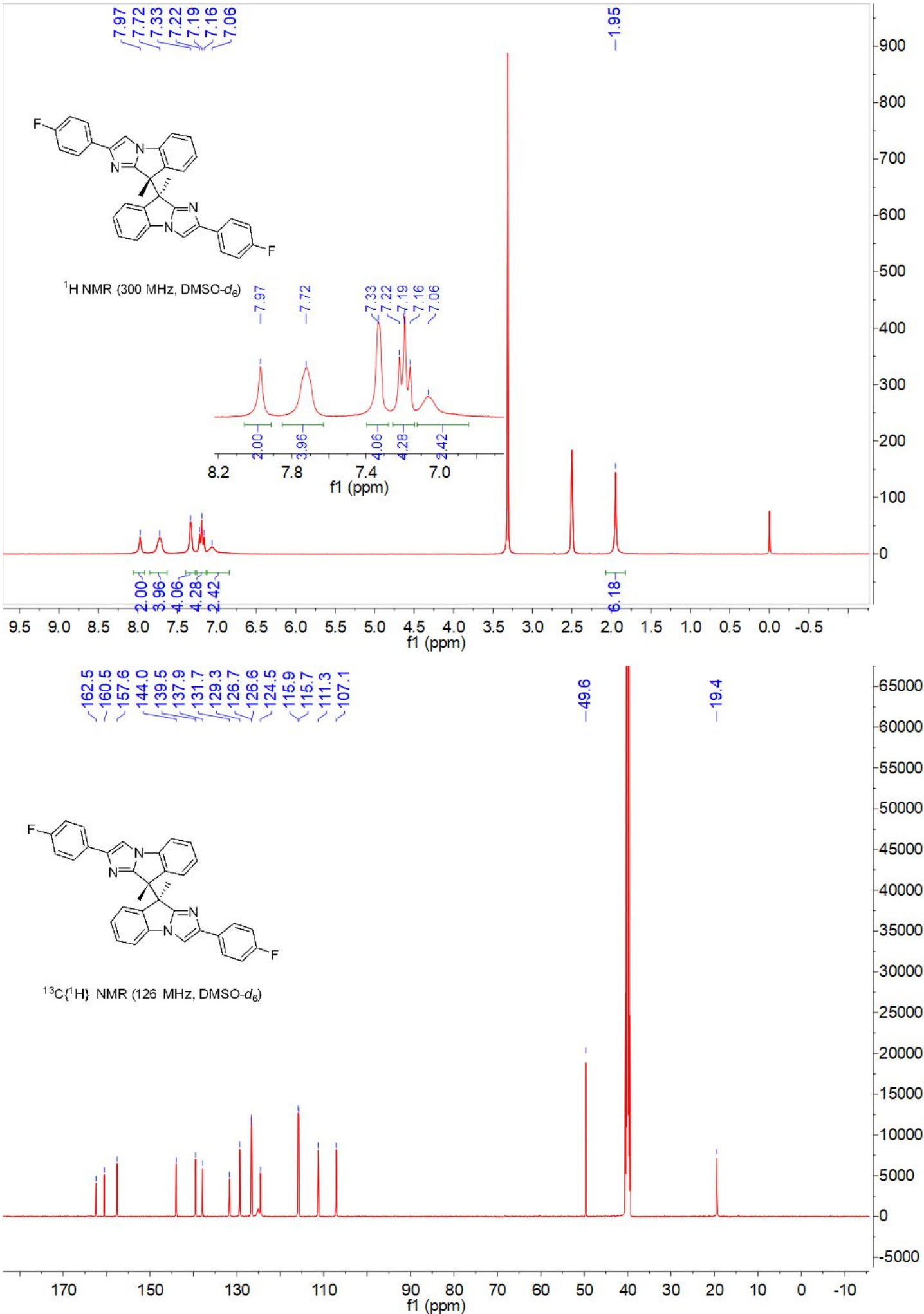


2,2'-bis(2,4-difluorophenyl)-9,9'-dimethyl-9H,9'H-9,9'-biimidazo[1,2-a]indole (dl-2t):

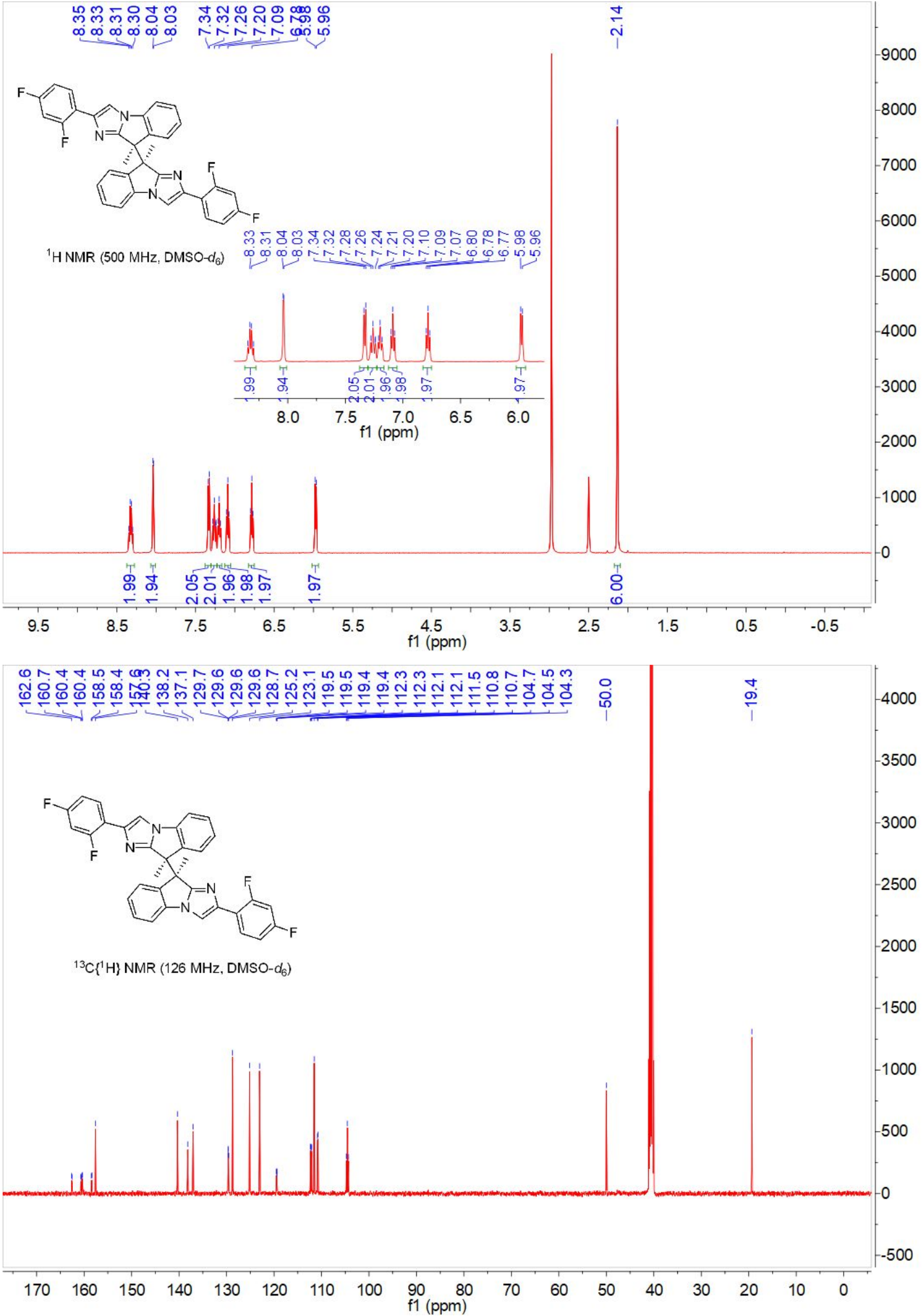


2,2'-bis(2,4-difluorophenyl)-9,9'-dimethyl-9H,9'H-9,9'-biimidazo[1,2-a]indole (meso-2t):

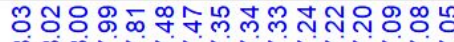

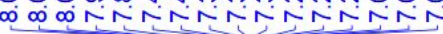
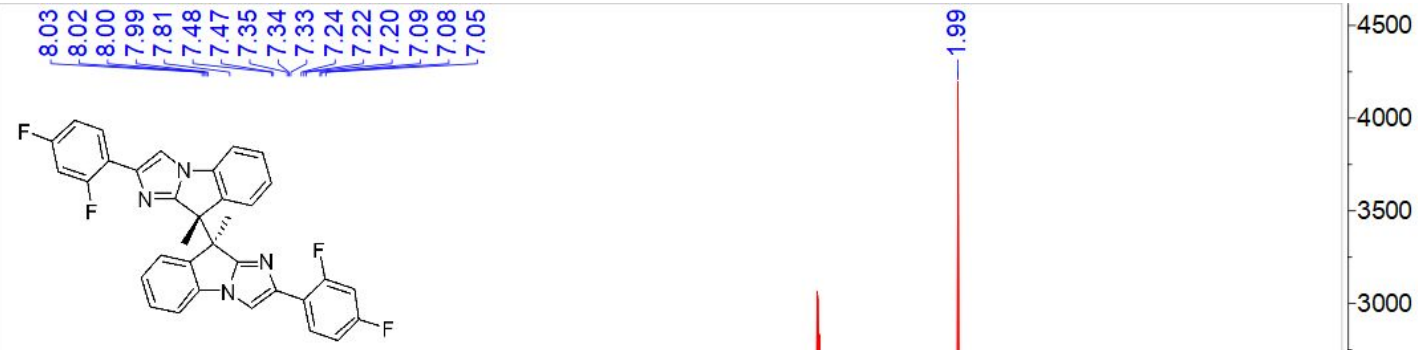

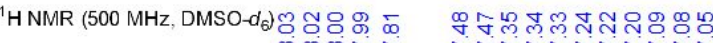

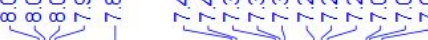
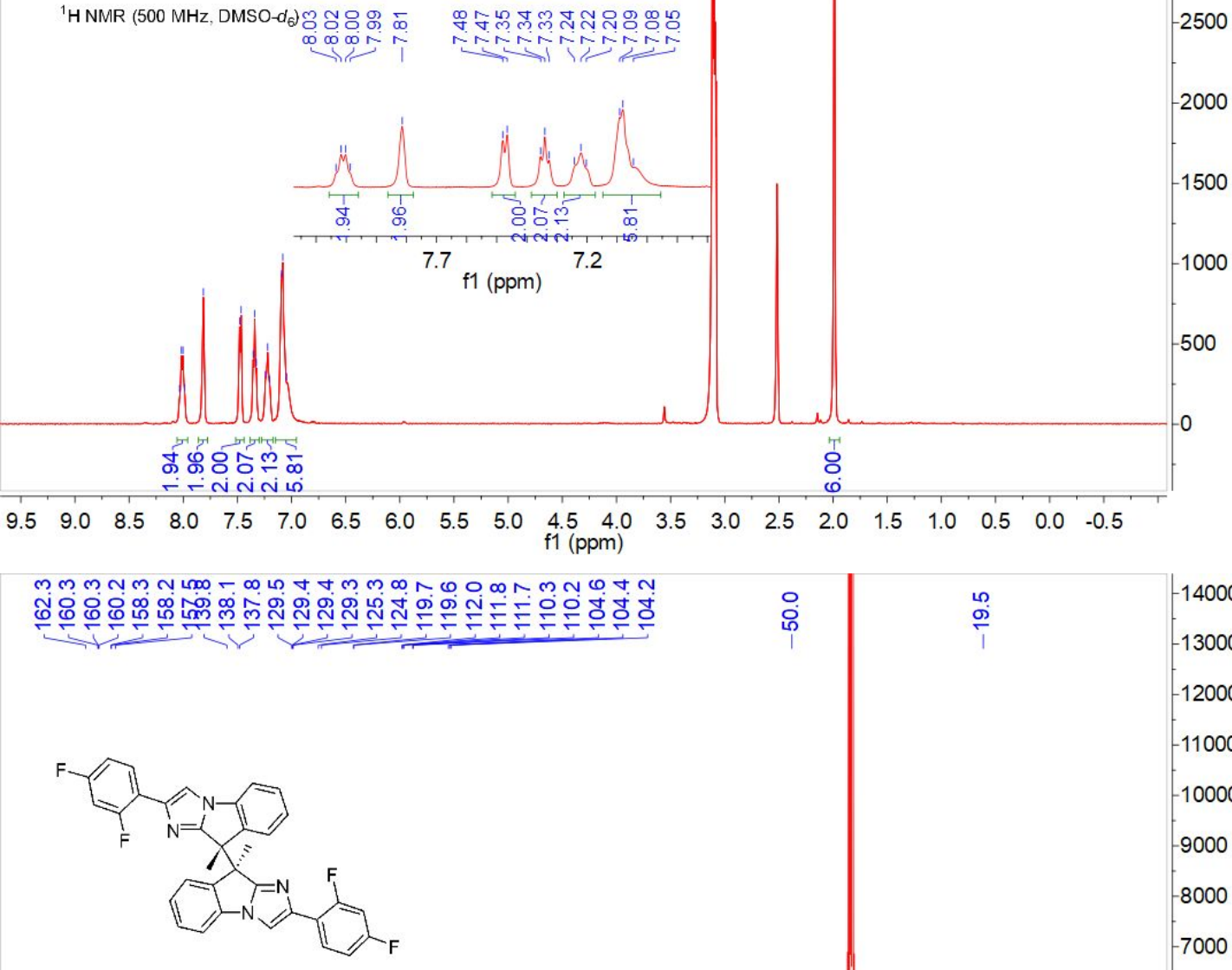

${ }^{13} \mathrm{C}\left\{{ }^{1} \mathrm{H}\right\}$ NMR (126 MHz, DMSO-d $)$

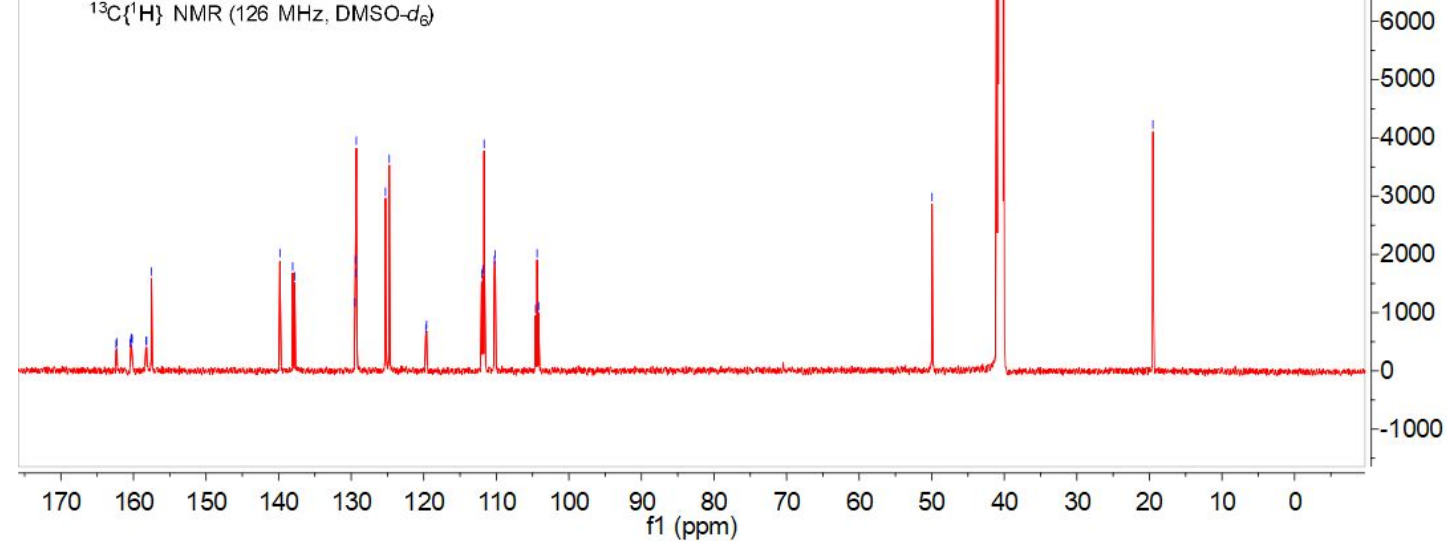


2,2'-bis(2-chlorophenyl)-9,9'-dimethyl-9H,9'H-9,9'-biimidazo[1,2-a]indole( $d l-2 u)$ :

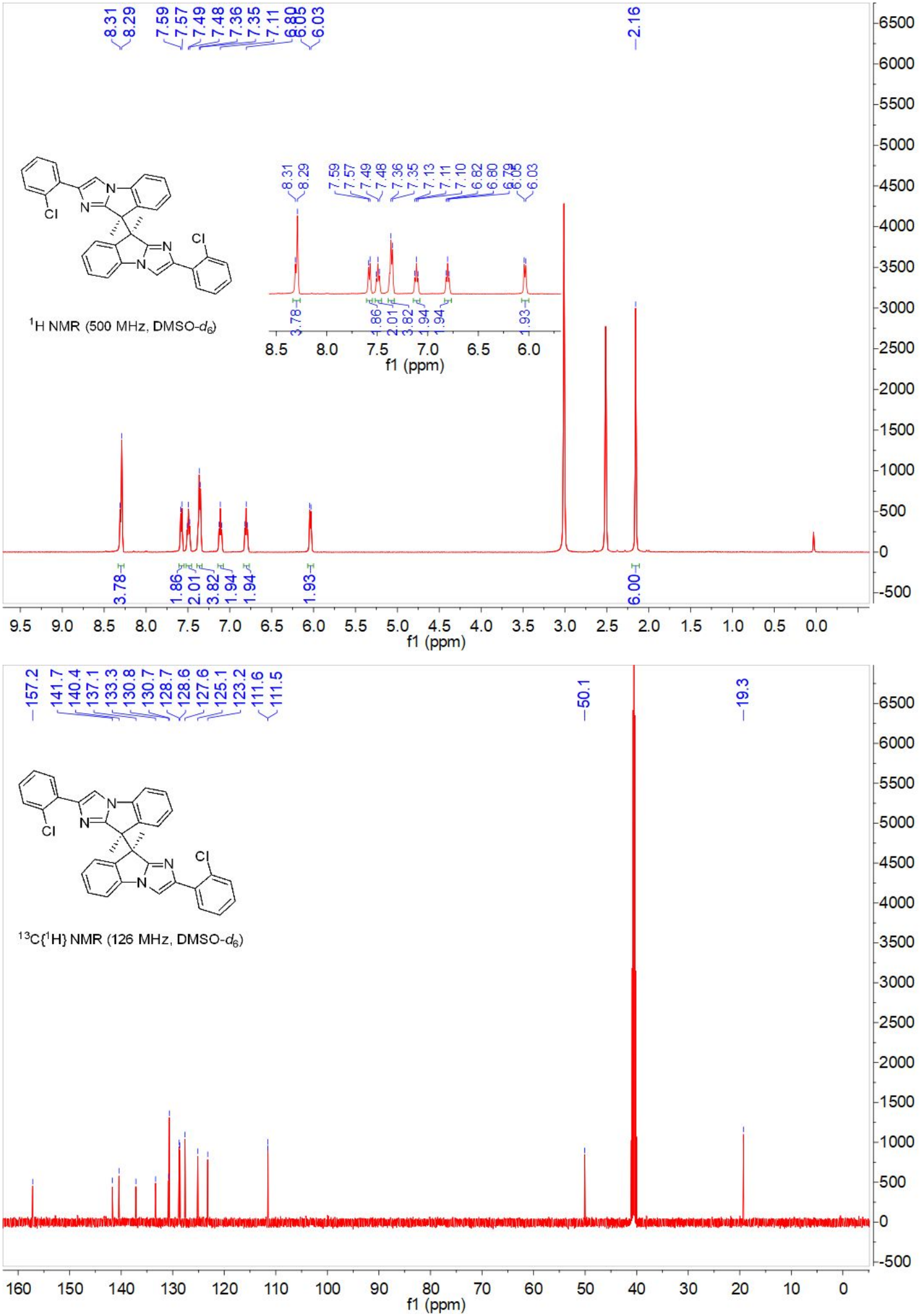


2,2'-bis(2-chlorophenyl)-9,9'-dimethyl-9H,9'H-9,9'-biimidazo[1,2-a]indole(meso-2u):

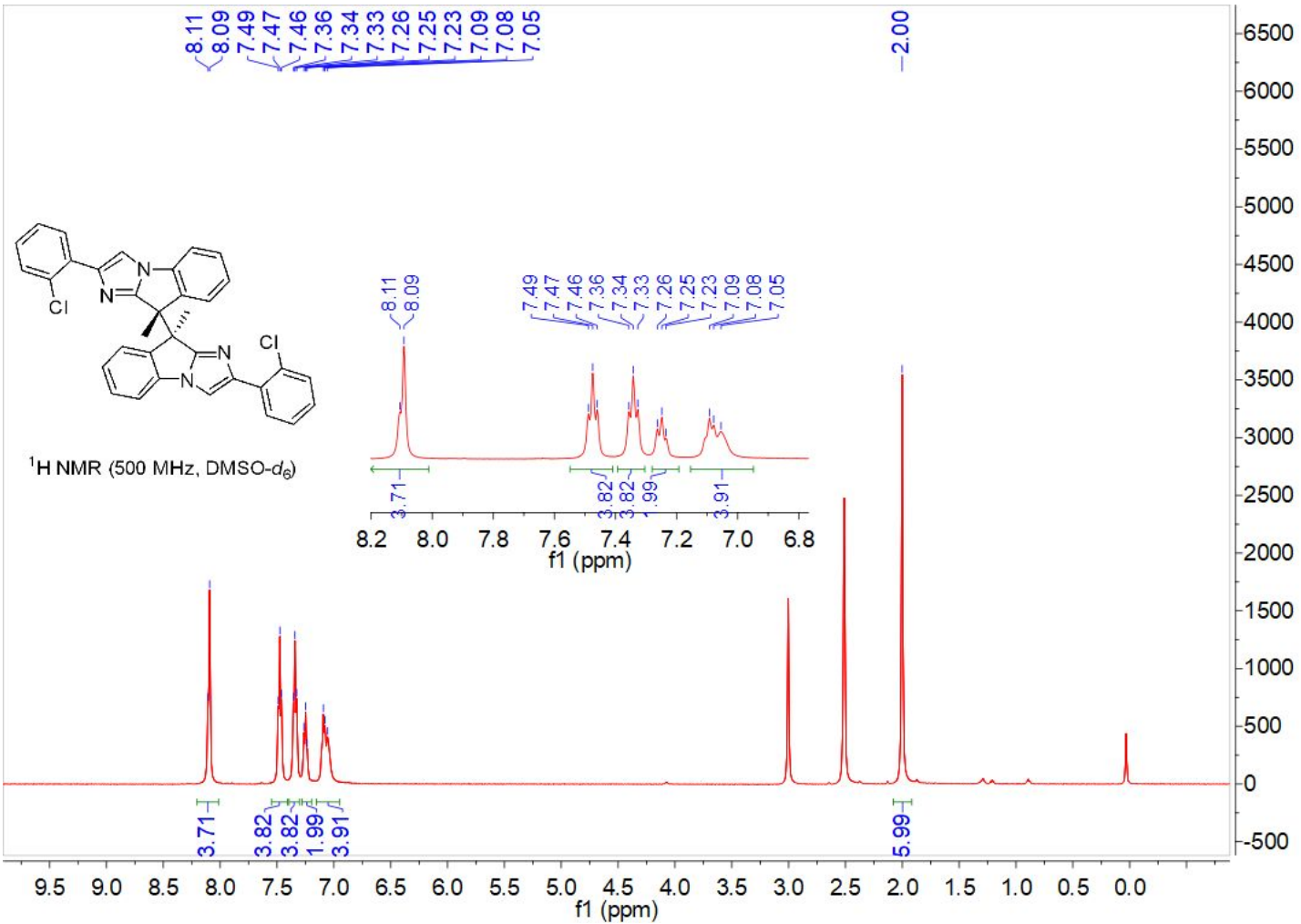

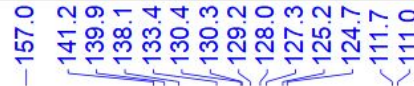
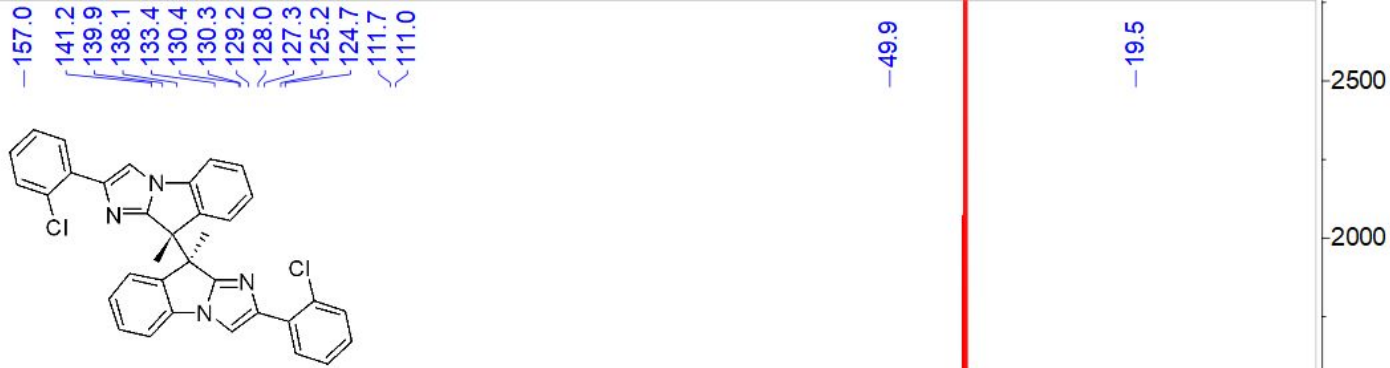

${ }^{13} \mathrm{C}\left\{{ }^{1} \mathrm{H}\right\}$ NMR (126 MHz, DMSO- $\left.d_{6}\right)$

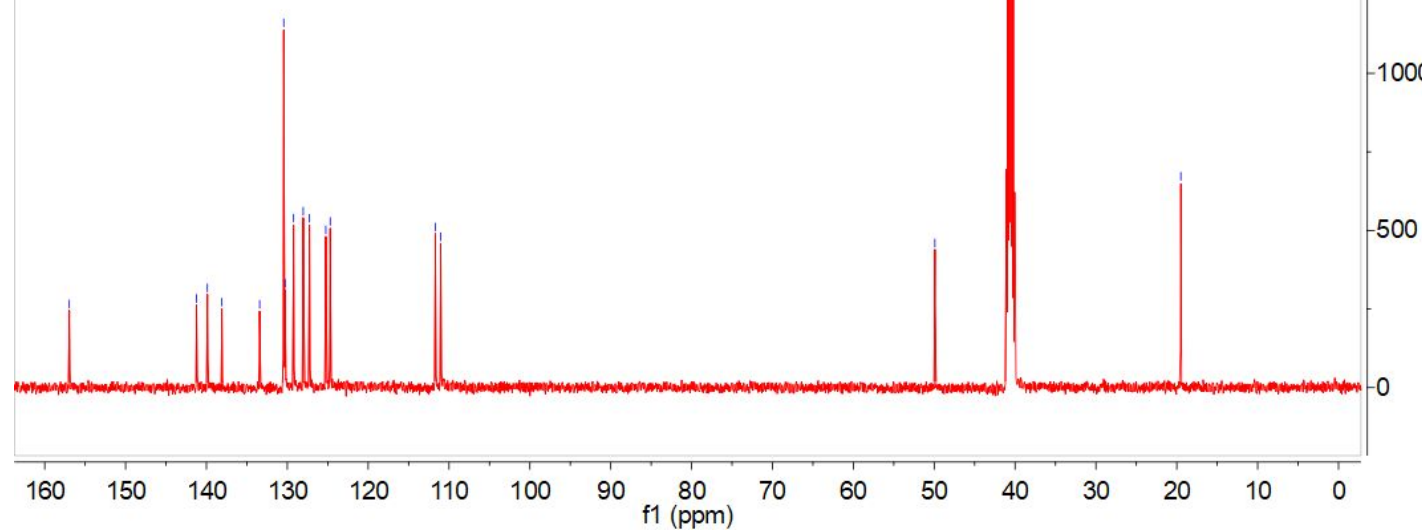


2,2'-bis(3-chlorophenyl)-9,9'-dimethyl-9H,9'H-9,9'-biimidazo[1,2-a]indole ( $d l-2 \mathrm{v})$ :

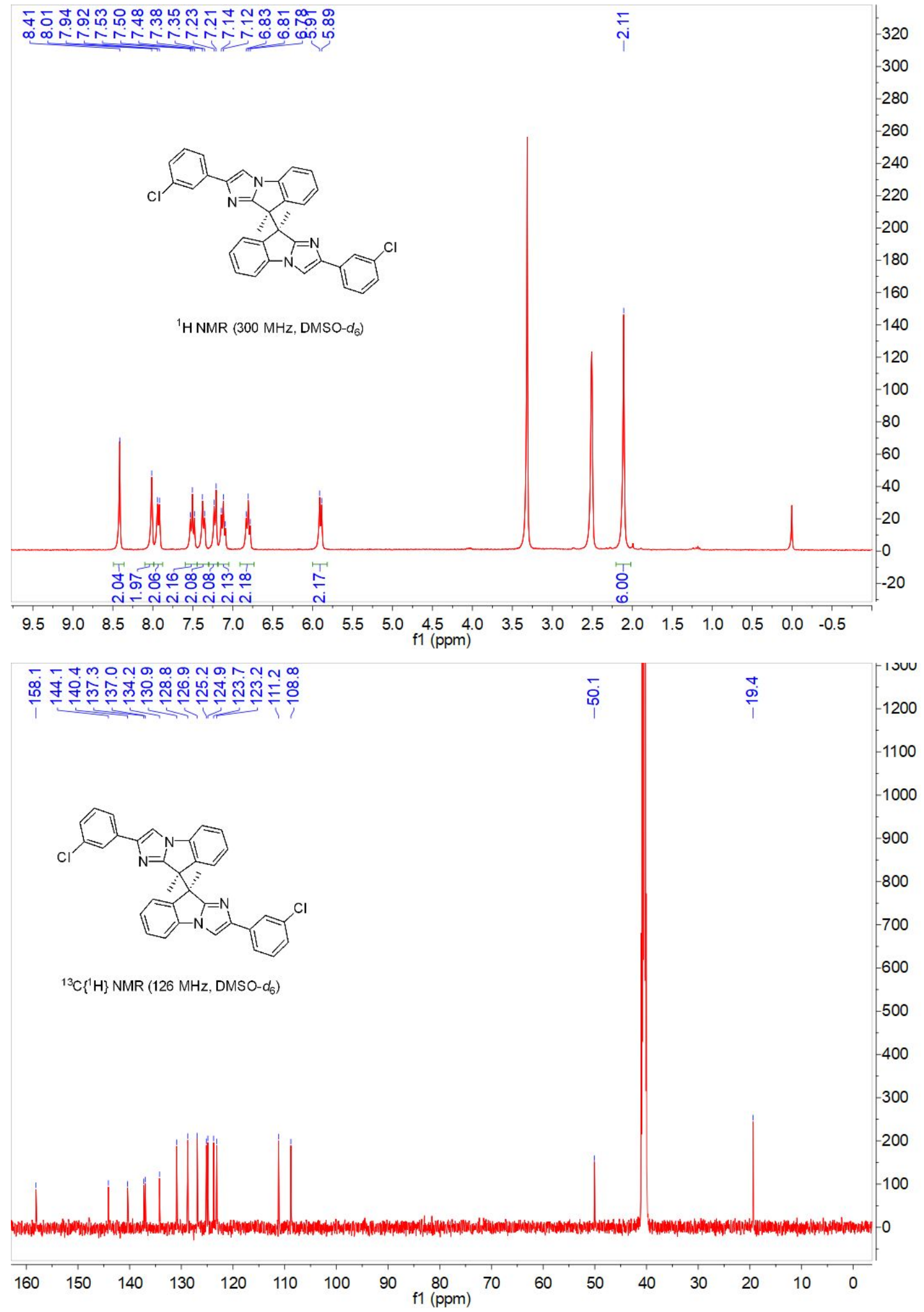


2,2'-bis(3-chlorophenyl)-9,9'-dimethyl-9H,9'H-9,9'-biimidazo[1,2-a]indole (meso-2v):

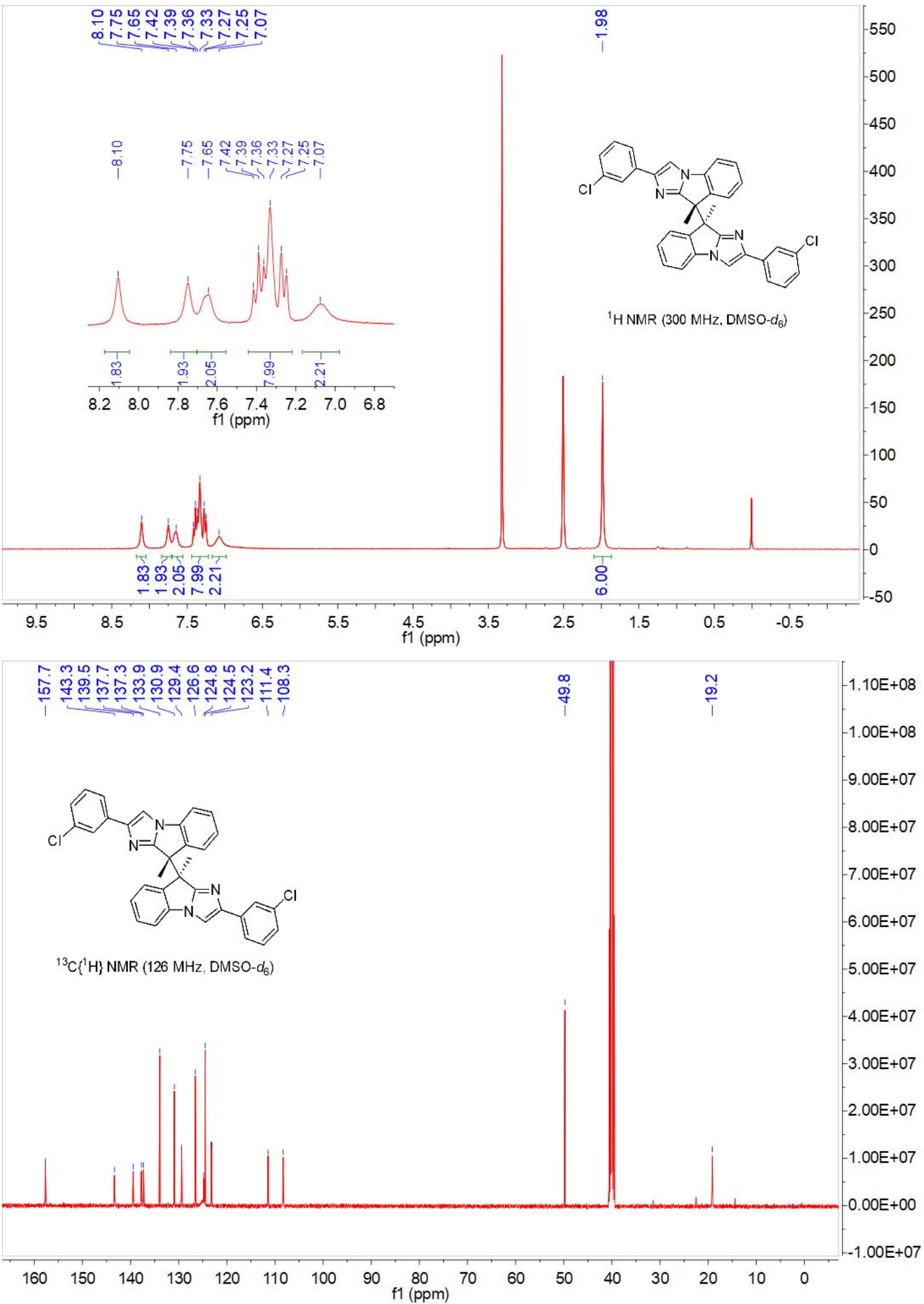


2,2'-bis(4-chlorophenyl)-9,9'-dimethyl-9H,9'H-9,9'-biimidazo[1,2-a]indole ( $\mathrm{dl}$-2w):

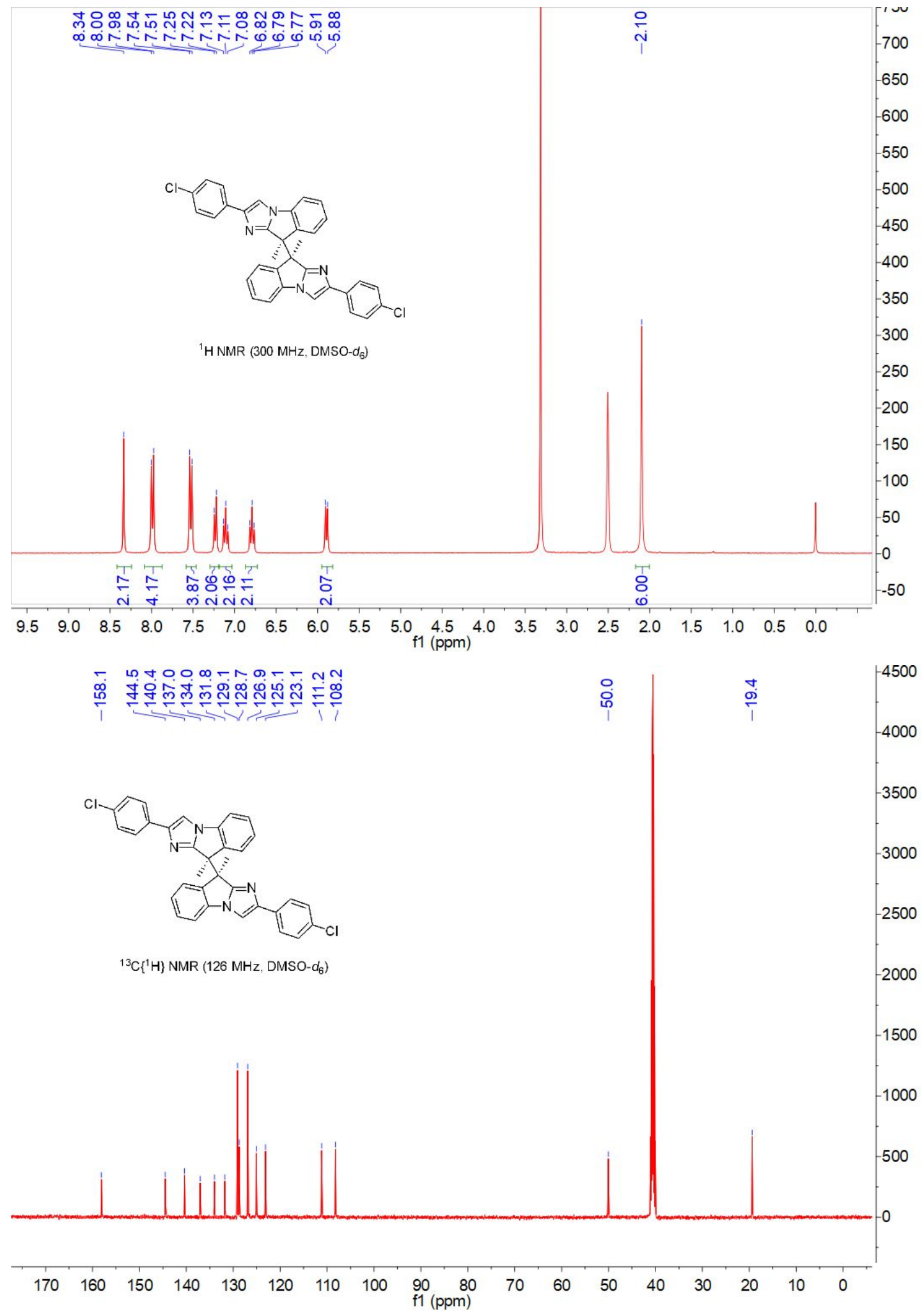


2,2'-bis(4-chlorophenyl)-9,9'-dimethyl-9H,9'H-9,9'-biimidazo[1,2-a]indole (meso-2w):

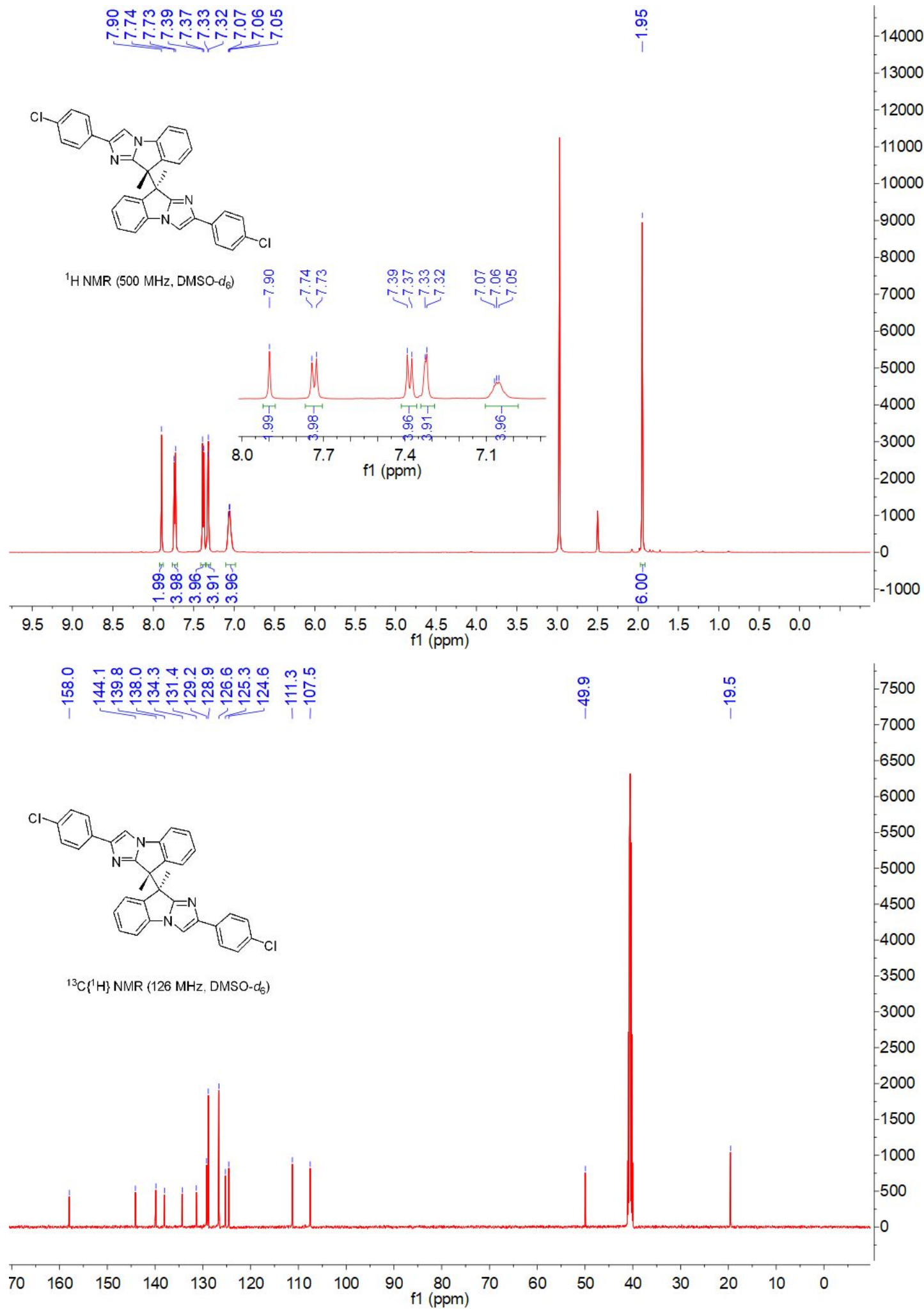


2,2'-bis(3,4-dichlorophenyl)-9,9'-dimethyl-9H,9'H-9,9'-biimidazo[1,2-a]indole( $d l-2 \mathrm{x})$ :

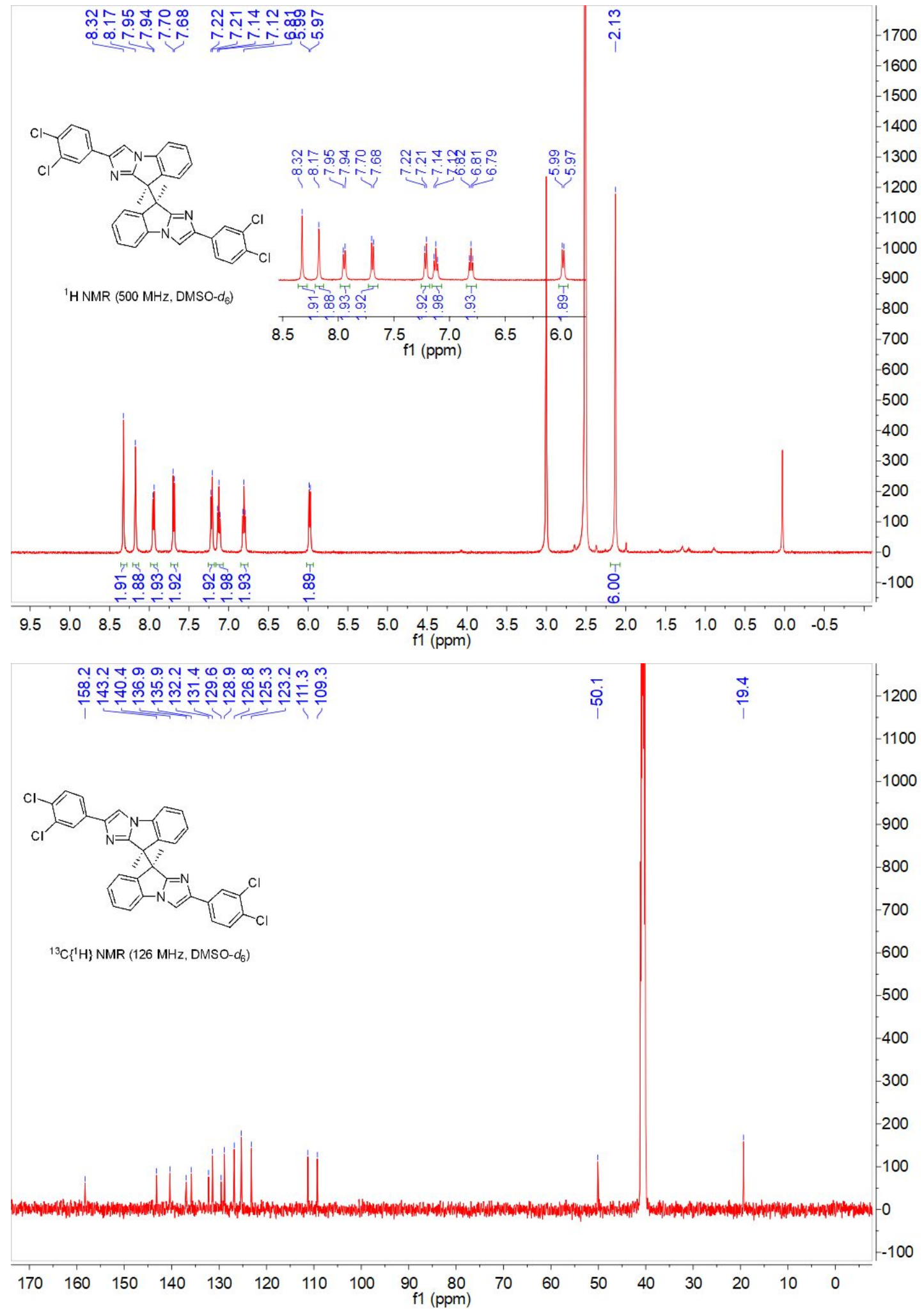


2,2'-bis(3,4-dichlorophenyl)-9,9'-dimethyl-9H,9'H-9,9'-biimidazo[1,2-a]indole(meso-2x):

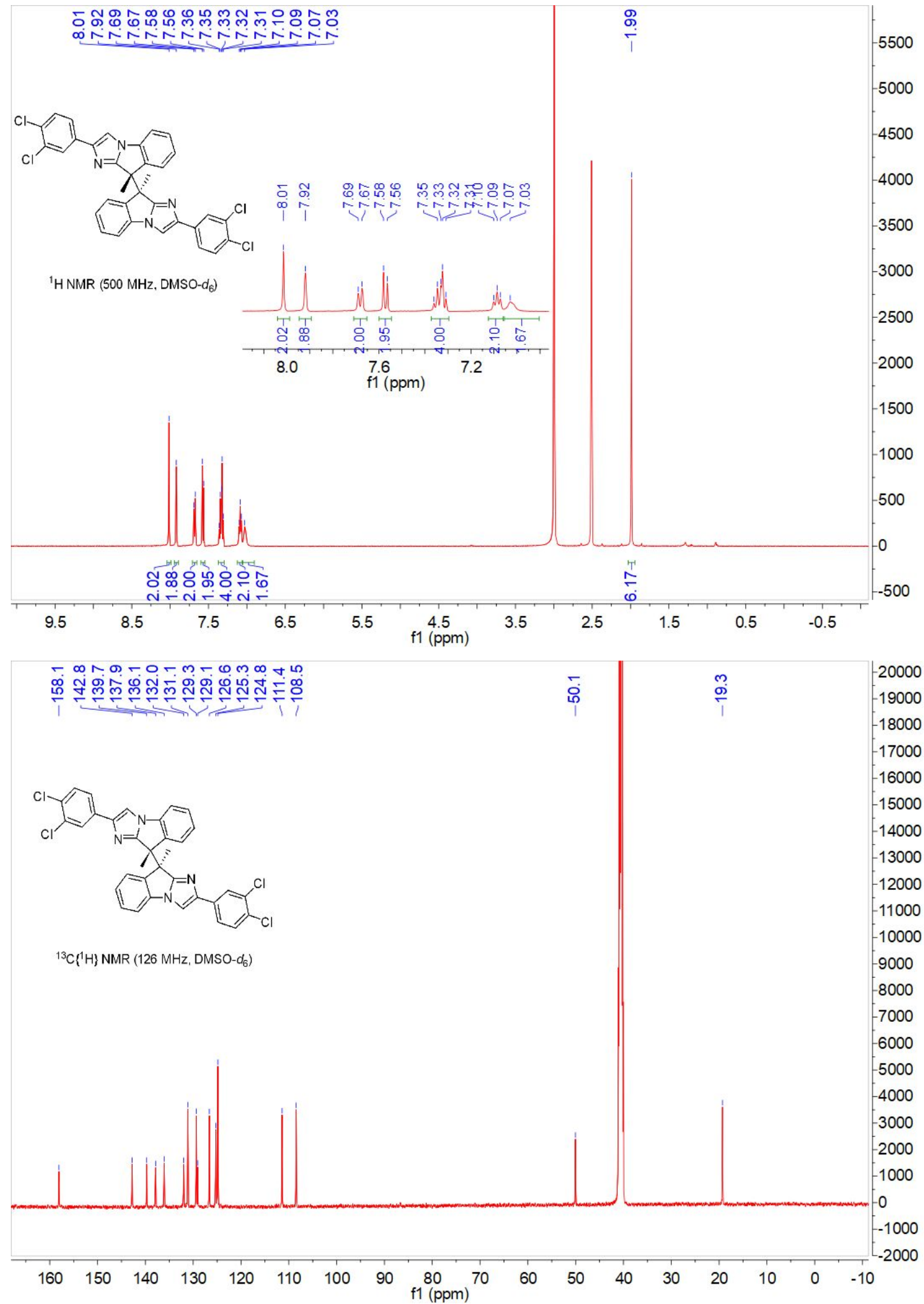


2,2'-bis(4-bromophenyl)-9,9'-dimethyl-9H,9'H-9,9'-biimidazo[1,2-a]indole (dl-2y):

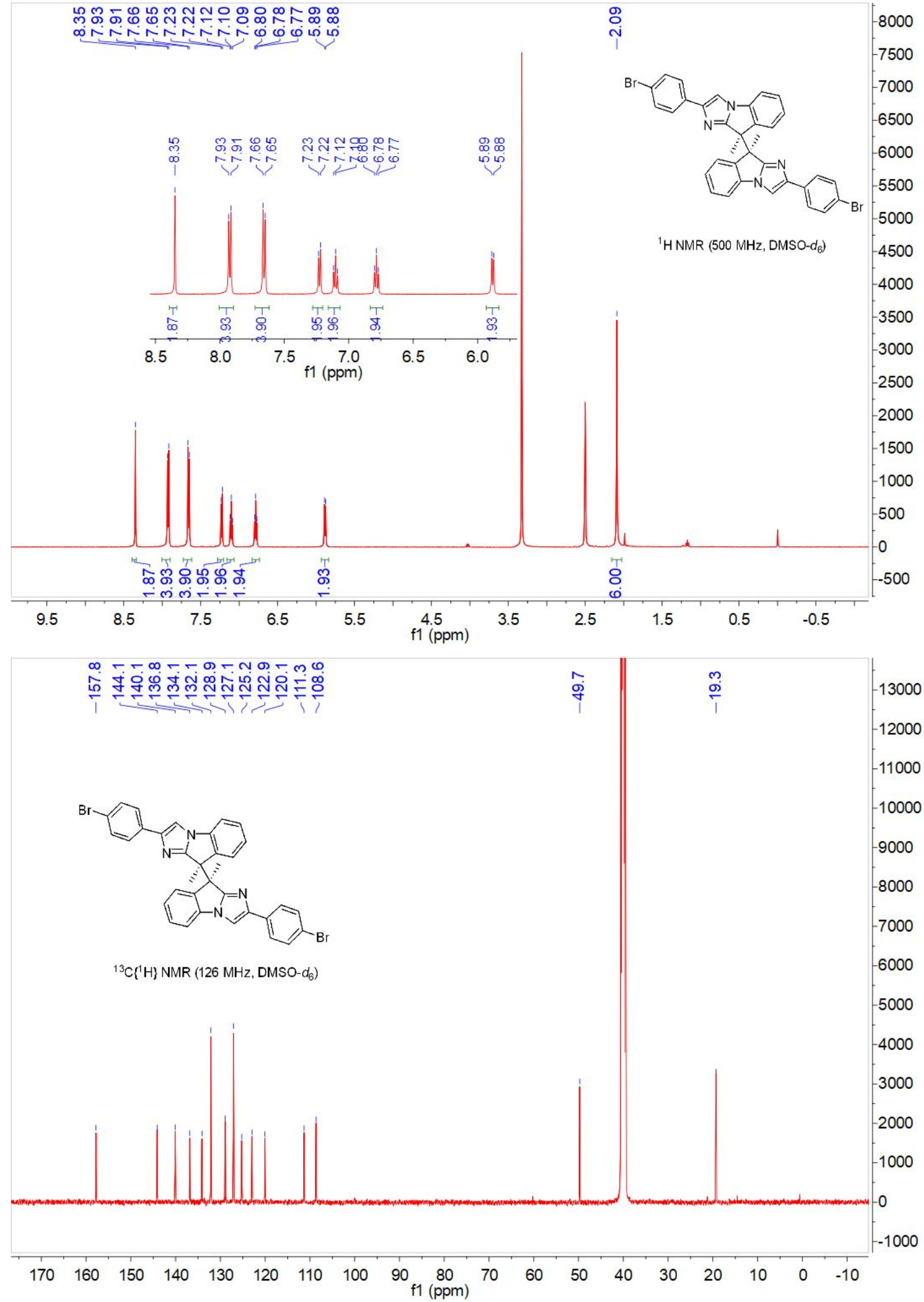


2,2'-bis(4-bromophenyl)-9,9'-dimethyl-9H,9'H-9,9'-biimidazo[1,2-a]indole (meso-2y):

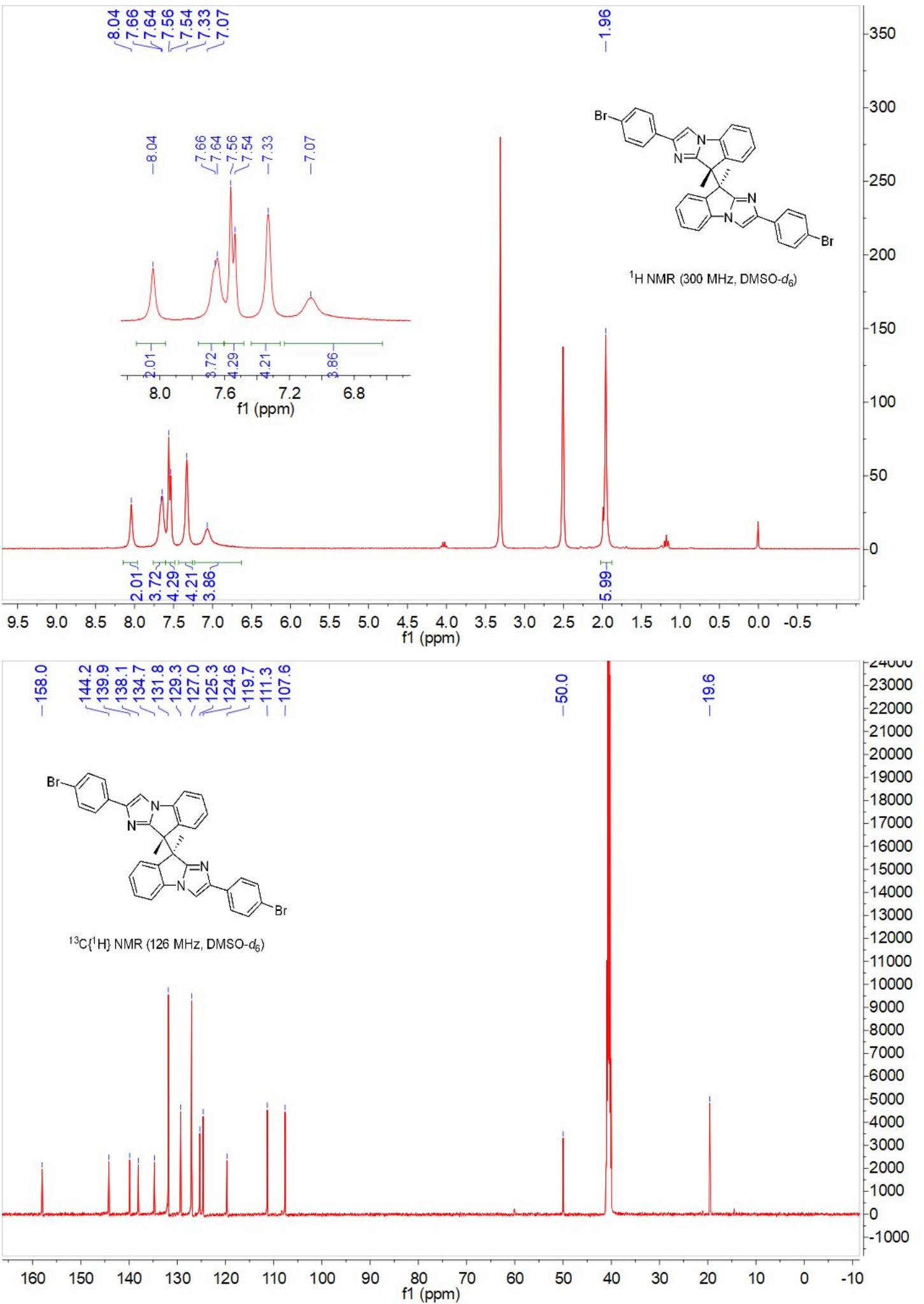


(E)-1-phenyl-2-(1,2,3,4-tetrahydro-9H-carbazol-9-yl)ethan-1-one O-acetyl oxime (3):

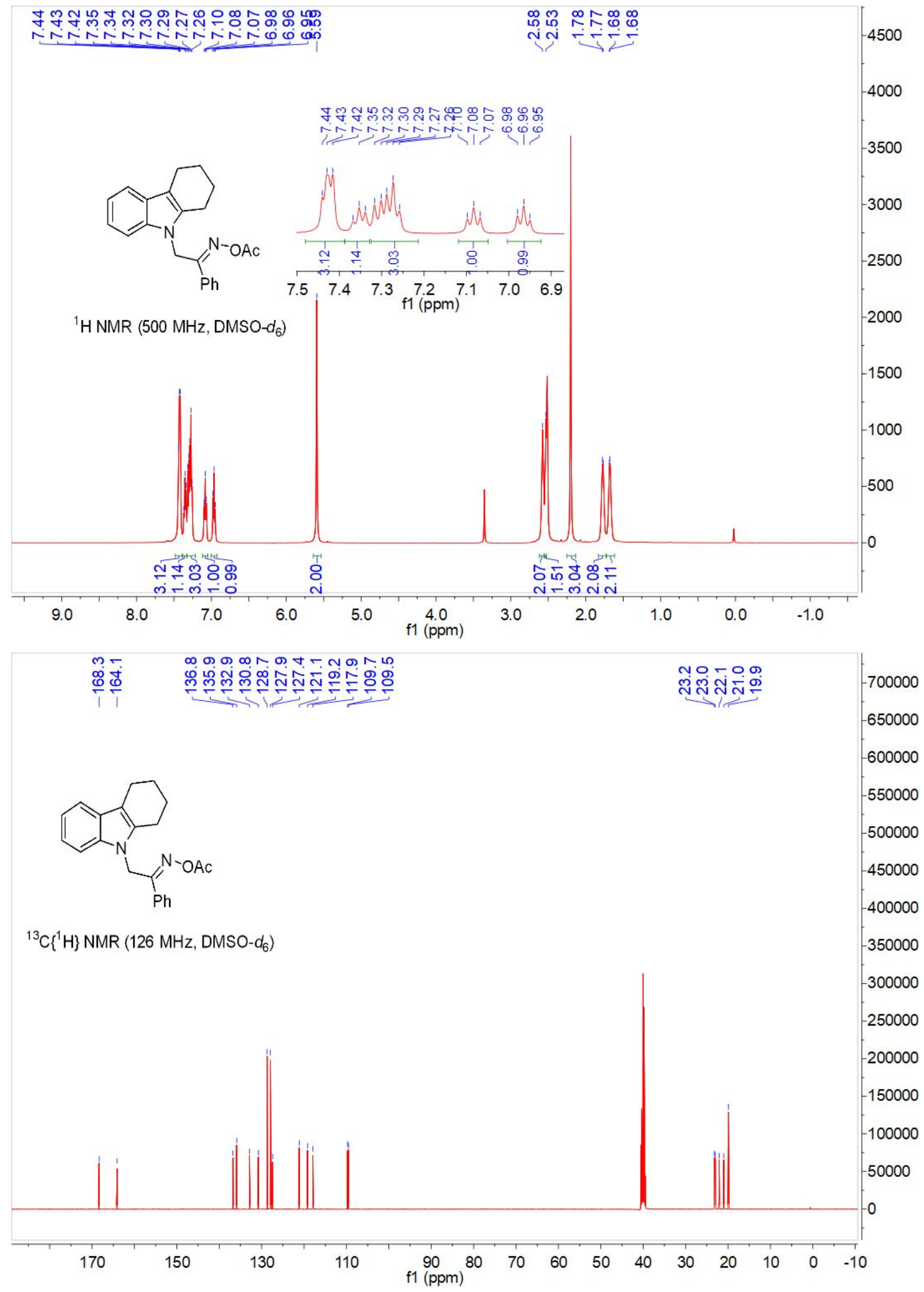


2-phenyl-4,5,6,7-tetrahydro-1H,7aH-imidazo[2,1-k]carbazol-7a-ol (4):

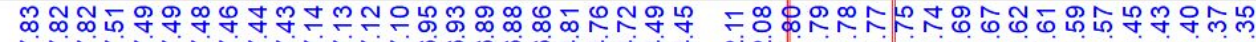

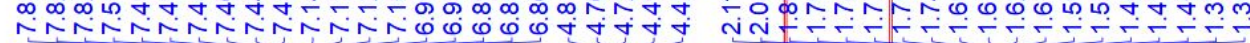<smiles>OC12CCCN=C(c3ccccc3)C13NCCC23</smiles>

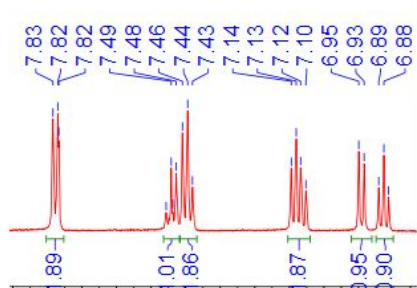

${ }^{1} \mathrm{H}$ NMR $\left(500 \mathrm{MHz}, \mathrm{DMSO}-\mathrm{d}_{6}\right){ }^{7.7} \mathrm{f1}(\mathrm{ppm})^{7.2}$

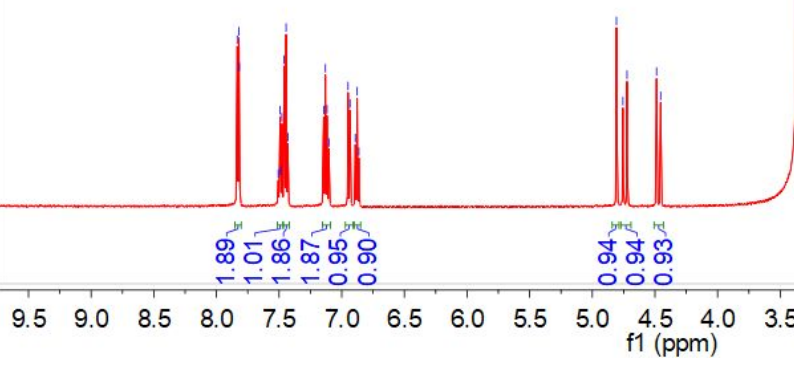

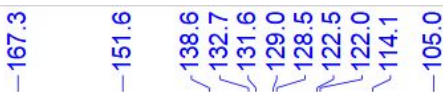<smiles>OC12CCCCC13N=C(c1ccccc1)CN3c1ccccc12</smiles>

${ }^{13} \mathrm{C}\left\{{ }^{1} \mathrm{H}\right\}$ NMR (126 MHz, DMSO- $\left.d_{6}\right)$

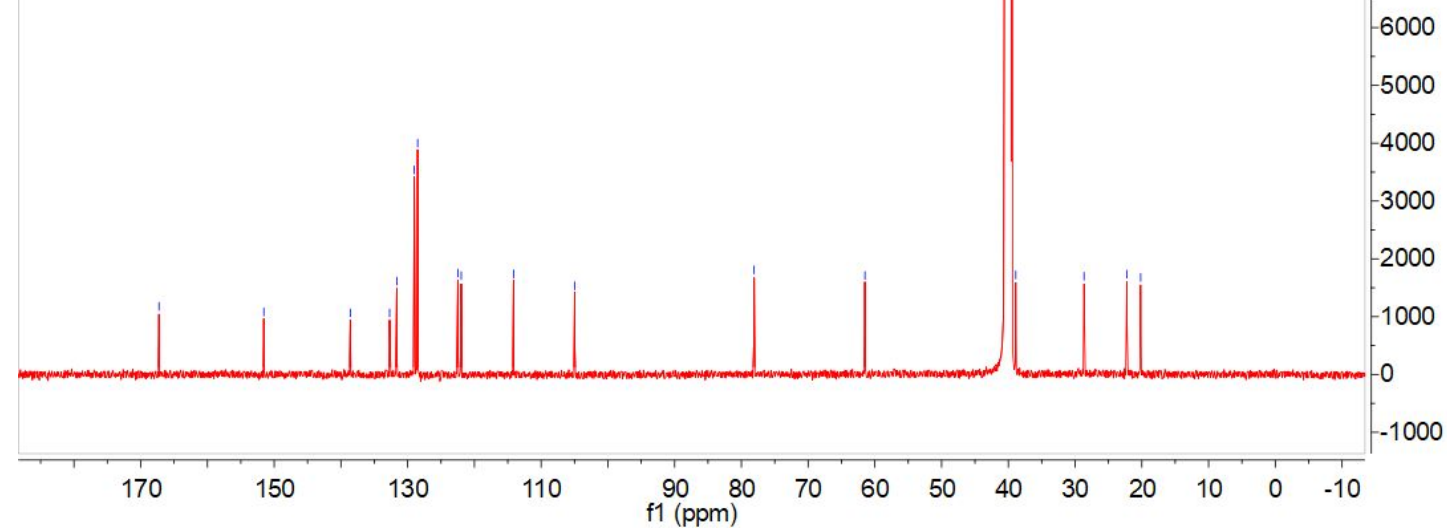


3. X-ray single crystal for compounds $\boldsymbol{d l - 2 a}$ 、 meso-2a and $\mathbf{4}$ (Thermal ellipsoids are set at $30 \%$ probability, The instrument used for the crystal measurement is ROD synergy-s.).

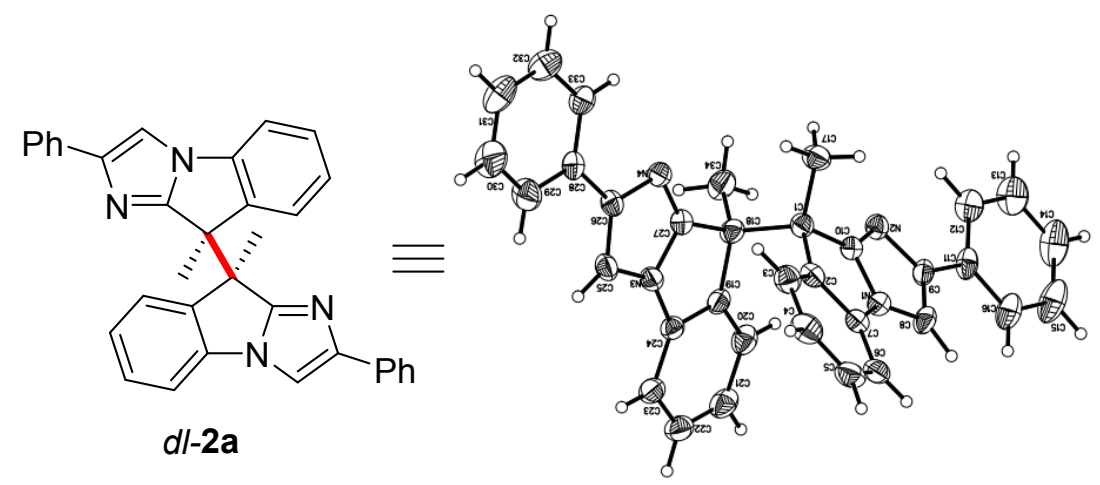

Figure 1. X-ray crystal structure of compounds dl-2a (CCDC-2053628). The crystal was grown from $\mathrm{MeOH}$ and DCM. $30 \mathrm{mg}$ of $\boldsymbol{d l}$-2a was dissolved in $\mathrm{MeOH}$ and DCM $(10 / 1,5.5 \mathrm{~mL})$ and the solvent was evaporated slowly in a room atmosphere.

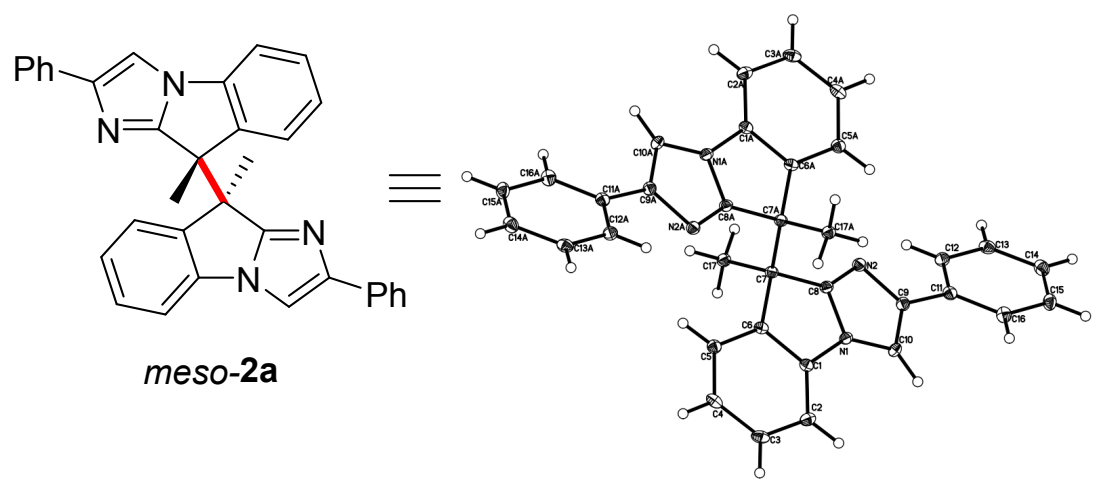

Figure 2. X-ray crystal structure of compounds meso-2a (CCDC-2053629). The crystal was grown from $\mathrm{MeOH}$ and DCM. $30 \mathrm{mg}$ of meso-2a was dissolved in $\mathrm{MeOH}$ and $\operatorname{DCM}(10 / 1,5.5 \mathrm{~mL})$ and the solvent was evaporated slowly in a room atmosphere.
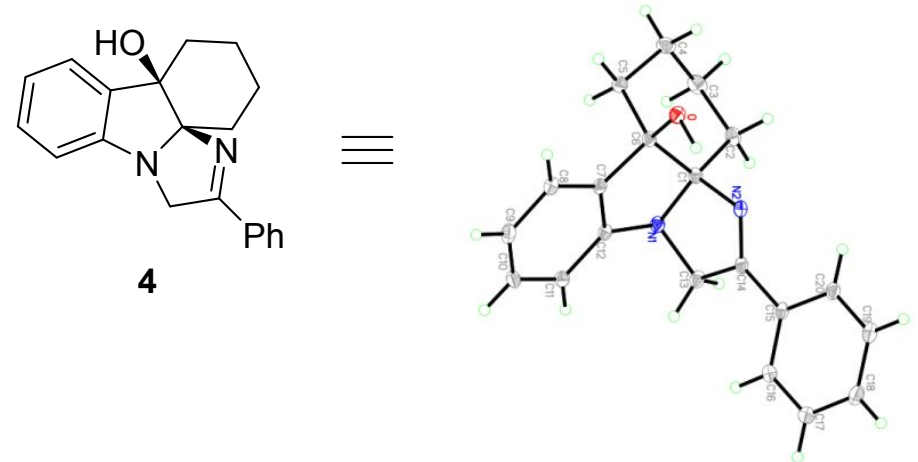

Figure 3. X-ray crystal structure of compounds 4 (CCDC-2053636). The crystal was grown from hexane and $\mathrm{Et}_{2} \mathrm{O} .30 \mathrm{mg}$ of 4 was dissolved in hexane and $\mathrm{Et}_{2} \mathrm{O}(2 / 1,6 \mathrm{~mL})$ and the solvent was evaporated slowly in a room atmosphere. 
Single crystal X-ray analysis Data of compounds dl-2a, meso-2a and 4.

\begin{tabular}{|c|c|c|c|}
\hline Compound & $d l-2 \mathrm{a}$ & meso-2a & 4 \\
\hline Formula & $\mathrm{C}_{34} \mathrm{H}_{26} \mathrm{~N}_{4}$ & $\mathrm{C}_{17} \mathrm{H}_{13} \mathrm{~N}_{2}$ & $\mathrm{C}_{20} \mathrm{H}_{20} \mathrm{~N}_{2} \mathrm{O}$ \\
\hline Formula weight & 490.59 & 245.29 & 304.38 \\
\hline Temperature $(\mathrm{K})$ & $293(2)$ & $293(2)$ & $293(2)$ \\
\hline Crystal colour & colourless & colourless & colourless \\
\hline Crystal system & monoclinic & monoclinic & monoclinic \\
\hline Space group & $\mathrm{P} 21 / \mathrm{C}$ & $\mathrm{P} 21 / \mathrm{C}$ & $\mathrm{P} 2 / \mathrm{n}$ \\
\hline $\mathrm{a}(\AA)$ & $22.010(4)$ & $9.3273(2)$ & $7.15030(10)$ \\
\hline $\mathrm{b}(\AA)$ & $8.5480(17)$ & $10.4481(2)$ & $10.24080(10)$ \\
\hline $\mathrm{c}(\AA)$ & $14.669(3)$ & $12.6645(3)$ & $20.8687(2)$ \\
\hline$\alpha\left(^{\circ}\right)$ & 90 & 90 & 90 \\
\hline$\beta\left(^{\circ}\right)$ & $108.98(3)$ & $91.517(2)$ & $93.6910(10)$ \\
\hline$\gamma\left({ }^{\circ}\right)$ & 90 & 90 & 90 \\
\hline $\mathrm{V}\left(\AA^{3}\right)$ & $2609.8(9)$ & $1233.76(5)$ & $1524.94(3)$ \\
\hline $\mathrm{Z}$ & 4 & 4 & 4 \\
\hline $\mathrm{Dc} / \mathrm{g} \mathrm{cm}^{-3}$ & 1.249 & 1.321 & 1.326 \\
\hline Wavelength & 0.71073 & 1.54178 & 1.54178 \\
\hline$\mu / \mathrm{mm}^{-1}$ & 0.074 & 0.610 & 0.646 \\
\hline $\mathrm{F}(000)$ & 1032 & 516 & 648 \\
\hline $\mathrm{R}$ (int) & 0.0370 & 0.0285 & 0.0638 \\
\hline Total reflections & 4791 & 2330 & 3063 \\
\hline Independent reflections & 2211 & 2159 & 2850 \\
\hline$\theta_{\min }<\theta<\theta_{\max }$ & $1.96<\theta<25.38$ & $4.743<\theta<70.461$ & $4.246<\theta<74.219$ \\
\hline $\mathrm{R} 1, \mathrm{wR} 2(\mathrm{I}>2 \sigma(\mathrm{I}))$ & $0.0660,0.1194$ & $0.0472,0.1333$ & $0.0384,0.1281$ \\
\hline R1, wR2 (for all data) & $0.1657,0.1583$ & $0.0499,0.1377$ & $0.0412,0.1313$ \\
\hline Goodness-of-fit on F2 & 1.001 & 1.003 & 1.003 \\
\hline
\end{tabular}

